\title{
Dietary assessment methods for micronutrient intake in infants, children and adolescents: a systematic review
}

\author{
Adriana Ortiz-Andrellucchi ${ }^{1}$, Patricia Henríquez-Sánchez ${ }^{1}$, Almudena Sánchez-Villegas, \\ Luis Peña-Quintana ${ }^{1}$, Michelle Mendez ${ }^{2}$ and Lluís Serra-Majem ${ }^{1 *}$ \\ ${ }^{1}$ Nutrition Research Group, Department of Clinical Sciences, Centre for Health Sciences, University of Las Palmas de Gran \\ Canaria, PO Box 550, 35080 Las Palmas de Gran Canaria, Spain \\ ${ }^{2}$ Center for Research in Environmental Epidemiology, Municipal Institute of Medical Research. Biomedical Research Park, \\ Barcelona, Spain
}

(Received 15 June 2009 - Revised 2 October 2009 - Accepted 1 November 2009)

A systematic literature search identified studies validating the methodology used for measuring the usual dietary intake in infants, children and adolescents. The quality of each validation study selected was assessed using a European micronutrient Recommendations Aligned-developed scoring system. The validation studies were categorised according to whether the study used a reference method that reflected short-term intake $(<7 \mathrm{~d})$, long-term intake $(\geq 7 \mathrm{~d})$ or used biomarkers. A correlation coefficient for each nutrient was calculated from the mean of the correlation coefficients from each study weighted by the quality of the study. Thirty-two articles were included in the present review: validation studies from infants (1-23 months); child preschool (2-5 years); children (6-12 years); adolescents (13-18 years). Validation of FFQ studies in infants and preschool children using a reference method that reflected short-term intake showed good correlations for niacin, thiamin, vitamins $\mathrm{B}_{6}$, $\mathrm{D}, \mathrm{C}, \mathrm{E}$, riboflavin, $\mathrm{Ca}, \mathrm{K}, \mathrm{Mg}, \mathrm{Fe}$ and $\mathrm{Zn}$ (with correlations ranging from 0.55 for vitamin $\mathrm{E}$ to 0.69 for niacin).Regarding the reference method reflecting short-term intake in children and adolescents, good correlations were seen only for vitamin $\mathrm{C}(r 0 \cdot 61)$ and Ca $(r 0.51)$. Using serum levels of micronutrient demonstrated that the $3 \mathrm{~d}$ weighed dietary records was superior to the FFQ as a tool to validate micronutrient intakes. Including supplement users generally improved the correlations between micronutrient intakes estimated by any of the dietary intake methods and respective biochemical indices.

Infants: Children: Adolescents: Dietary assessment methods: Systematic review: Validation: Micronutrients

Growth in children from birth through adolescence is an extremely complex process. It is influenced not only by the genetic make-up of the individual but also by environmental factors, medical illnesses and nutritional status ${ }^{(1)}$. Dietary assessments among infants and preschool children are complicated by the facts that dietary habits change rapidly in infancy, parents may share the responsibility for the child with other adults, e.g. in day-care, and finally not all food served to the infants are consumed, resulting in their disposal ${ }^{(2)}$.

In the school age years, children experience enormous cognitive, emotional and social growth and development. Children transition from consuming most food intake under adult control and supervision to taking increasing responsibility for their food choices. The cognitive abilities required to self-report food intake include an adequately developed concept of time, a good memory and attention span and knowledge of the names of food ${ }^{(3,4)}$. The need for adult assistance in dietary reporting is also driven by the limited scope of the child's experience and knowledge of food preparation ${ }^{(5)}$.
During adolescence, children undergo profound biological, emotional, social and cognitive changes to reach adult maturity. Adolescents' need for energy and all nutrients significantly increases to support the rapid rate of growth and development. Moreover, although appetite and food intake tend to increase, psychosocial characteristics often lead to the development of high-risk nutritional behaviours such as excessive dieting, adoption of fad diets or excessive alcohol consumption. The high prevalence of overweight and obesity, eating disorders, adolescent pregnancy and the lack of consumption of five fruits and vegetables a day constitute some of the nutritional challenges facing adolescents ${ }^{(6)}$.

Research conducted as part of the European Commission's European micronutrient Recommendations Aligned Network of Excellence ${ }^{(7)}$ has focused on extensive literature reviews addressing the validation of methods used to assess intake of micronutrients, $n-3$ fatty acids and of special population groups, including pregnant women, infants, children, adolescents and elderly people. In this review, studies validating

Abbreviations: BM, biomarker; EDR, estimated dietary records; 24HR, 24h dietary recall; WDR, weighed dietary records; YAQ, Youth/Adolescent Questionnaire. On behalf of EURRECA's RA.1.1 'Intake Methods' members: Serra-Majem L (Coordinator), Cavelaars A, Dhonukshe-Rutten R, Doreste JL, Frost-Andersen L, García-Álvarez A, Glibetic M, De Groot L, De Vries J, Gurinovic M, Henríquez-Sánchez P, Naska A, Ngo J, Novakovic R, Ortiz-Andrellucchi A, Øverby NC, Pijls L, Ranic M, Ribas-Barba L, Ristic-Medic D, Román-Viñas B, Ruprich J, Saavedra-Santana P, Sánchez-Villegas A, Tabacci G, Tepsic J, Trichopoulou A, van 't Veer P, Vucic V, Wijnhoven TMA.

* Corresponding author: Lluis Serra-Majem, fax +34928 453475, email lserra@dcc.ulpgc.es 
dietary methods for assessing micronutrient intake in infants, children and adolescents are presented.

\section{Material and methods}

The research question applied to the systematic review was 'which dietary methods are reliable for the assessment of micronutrient intake in infants, children and adolescents?' The main stages of the review are illustrated in Fig. 1. The review included English, Spanish, French, Italian, Portuguese and German articles, without limits on time frame or country published before April 2008. Stage 1 of the review involved searching for publications using electronic databases (MEDLINE and EMBASE). The MeSH terms used in the general search were: nutritional assessment, diet, nutritional status, dietary intake, food intake, validity, validation study, reproducibility, replication study, correlation coefficient and correlation study in the title and abstract. As a second specific search, the following words were included: infants (1-23 months), preschool child (2-5 years), children (6-12 years), adolescents (13-18 years), 'dietary assessment', 'dietary intake', 'nutrition assessment', 'diet quality', reliability, reproducibility, validit* and correlate* as free text in the title and abstract. Additional publications were identified from references published in the original papers. At stage 2 of the review, the titles and abstracts were analysed by two independent reviewers and the exclusion criteria were applied (Table 1). At stage 3, studies that fulfilled the inclusion criteria were analysed for relevance to the research question.

The selected studies were then classified into three different types according to the reference method applied in the validation studies: (1) reference method assessing intake of $<7 \mathrm{~d}$ (including $24 \mathrm{~h}$ dietary recall $(24 \mathrm{HR})$, estimated dietary records (EDR) and weighed dietary records (WDR)), classified as reflecting short-term intake; (2) reference method assessing intake of $\geq 7 \mathrm{~d}$, reflecting more long-term intake; (3) reference method that employed the use of a biomarker (BM). Furthermore, the different studies included in this review were scored according to a quality score system developed by European micronutrient Recommendations Aligned. The studies were rated according to the sample size, the statistics used to validate the method, the procedure of data collection, the consideration or not of seasonality and the inclusion or not of vitamin supplement use. (For details see the article in this supplement 'Evaluating the quality of dietary intake validation studies'). A total score was calculated according to the mean of the correlation coefficients weighted by the quality score of the validation study. It was considered a poor method for assessing specific nutrient intake when the correlation between

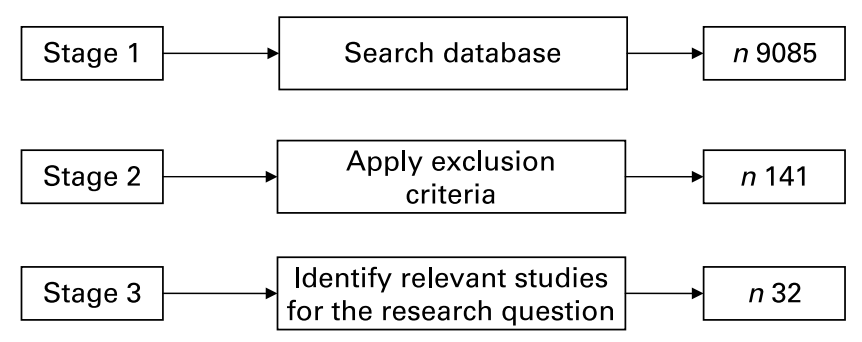

Fig. 1. Main stages of the systematic review process.
Table 1. Inclusion and exclusion criteria

Inclusion criteria
1. Studies on micronutrient intake in infants, children and adolescents
2. Validation study in human subjects
Exclusion criteria
1. Studies describing the content of foods in nutrients, additives or
contaminants.
2. Studies exclusively focused on diseased or institutionalised
persons.
3. Articles presenting reference values for food consumption, nutrient
intake, biochemical markers and anthropometric measurements.
4. Articles establishing associations between food consumption,
nutrient intake, biological variables, biochemical markers and
anthropometric measurements.
5. Studies relating diseases to food consumption or nutrient intake.
6. Intervention studies and other therapeutic studies with nutrients or
drugs related to the metabolism of these nutrients.
7. Calibration studies and those discussing statistical methods.
8. Studies evaluating the physiological effects of foods, nutrients and
in relation to their genetic determinants.
9. Studies in animals.
10. Studies written in other languages than English, Spanish, French,
Italian, Portuguese and German and those without abstract.

methods was $<0 \cdot 30$. Methods whose correlations were between 0.30 and 0.50 were regarded as acceptable for assessing nutrient intake. Good methods were those whose correlations were between 0.51 and 0.70 , and finally, when the correlation was $>0.70$ the method was considered very good.

\section{Results}

A total of thirty-two publications ${ }^{(2,8-39)}$ were selected for inclusion, with information on each validation study, ordered by publication year, summarised in Table 2 . Fifteen of the publications showed results from European countries (Norway, Greece, Belgium, Italy, Denmark, United Kingdom and Finland), fifteen from American countries (United States of America, Brazil and Canada), one study from Australia and one study from New Zealand. The number of participants varied from 17 to 741 in the selected studies. In eight of the studies presented $^{(11,13-16,26,34,36)}$, only one type of micronutrient was analysed, while in the rest of the publications included in this review, correlations for a wide variety of micronutrients were observed, and a total of twenty micronutrients were analysed. Tables 3 and 4 show information on the correlation between methods and other statistics in the validation studies in infants, children and adolescents for twelve vitamins and eight minerals, respectively.

\section{Infants}

This group included infants aged 1-23 months. Of the thirtytwo articles included in the present review, seven showed data on the validation of methods used to assess micronutrient intake in infants ${ }^{(2,8,9,17,21,22,24)}$. Evaluating the quality of these validation studies resulted in quality scores ranging from 2.5 to 5 . All the studies evaluated micronutrient intake in infants using a FFQ, and only one article applied four $24 \mathrm{HR}$ as an additional dietary assessment method ${ }^{(22)}$. Different FFQ were validated for which wide variations in 


\begin{tabular}{|c|c|c|c|c|c|}
\hline $\begin{array}{l}\text { Author/year } \\
\text { publication } \\
\text { and country }\end{array}$ & Participants/age group & Dietary method & Reference method & Micronutrient & Conclusions \\
\hline $\begin{array}{l}\text { Marriott et al. } \\
\qquad(2008)^{(8,9)} \text { UK }\end{array}$ & $\begin{array}{l}\text { Fifty infants aged } 6 \text { months } \\
\& \text { fifty infants aged } \\
12 \text { months } \\
\text { Infants }\end{array}$ & $\begin{array}{l}\text { FFQ Previous } 7 \text { ( } 6 \text { months) or } 28 \\
\text { (12 months) days intake; thirty- } \\
\text { four items at } 6 \text { months (including } \\
\text { ten categories of commercial } \\
\text { baby foods); seventy-eight items } \\
\text { at } 12 \text { months } \\
\text { Portion size estimated using } \\
\text { household measures. Exact fre- } \\
\text { quencies Interviewer-adminis- } \\
\text { tered }\end{array}$ & $\begin{array}{l}4 \mathrm{~d} \text { weighed dietary records } \\
\text { (within } 15 \text { days following } \\
\text { FFQ completion) } \\
\text { For breast-fed infants, the } \\
\text { length of each feeding } \\
\text { was recorded. }\end{array}$ & $\begin{array}{l}\mathrm{Na}, \mathrm{K}, \mathrm{Ca}, \mathrm{Mg}, \mathrm{P} . \mathrm{Fe}, \mathrm{Zn}, \\
\mathrm{Cu}, \text { retinol, vitamins } \mathrm{D}, \mathrm{E}, \\
\mathrm{C}, \mathrm{B}_{6}, \mathrm{~B}_{12} \text {, thiamin, } \\
\text { riboflavin, niacin, folic acid }\end{array}$ & $\begin{array}{l}\text { The interviewer-administered FFQ } \\
\text { are useful tools for assessing } \\
\text { energy and nutrient intakes of } \\
\text { healthy infants at } 6 \text { and } \\
12 \text { months. }\end{array}$ \\
\hline $\begin{array}{l}\text { Holmes et al. } \\
\qquad(2008)^{(10)} \text { UK }\end{array}$ & $\begin{array}{l}124 \text { children aged } \\
2-17 \text { years } \\
\text { Preschool children } \\
\text { Children } \\
\text { Adolescents }\end{array}$ & $\begin{array}{l}\text { (i) Four multipass } 24 \mathrm{~h} \text { dietary } \\
\text { recalls. Portion size in household } \\
\text { measures or using colour photo- } \\
\text { graphs Interviewer-administered } \\
\text { (ii) } 4 \mathrm{~d} \text { food checklist or daily } \\
\text { record of foods eaten; portion } \\
\text { size in household measures } \\
\text { (iii) } 4 \mathrm{~d} \text { semi-weighed method } \\
\text { Food consumed at home } \\
\text { weighed and estimated intake } \\
\text { allocated to members; sup- } \\
\text { plemented with diary for con- } \\
\text { sumption away from home }\end{array}$ & $\begin{array}{l}4 \mathrm{~d} \text { weighed dietary records } \\
\text { (weighed inventory). } \\
\text { Foods consumed and left- } \\
\text { overs weighed for each } \\
\text { participant individually. }\end{array}$ & $\begin{array}{l}\mathrm{Ca} \text {, Fe, retinol, thiamin, } \\
\text { vitamin C }\end{array}$ & $\begin{array}{l}\text { Four } 24 \mathrm{~h} \text { recalls were } \\
\text { recommended as the most } \\
\text { appropriate method to estimate } \\
\text { dietary intakes in low-income } \\
\text { households based in part on } \\
\text { data to be presented in future } \\
\text { publications. }\end{array}$ \\
\hline $\begin{array}{l}\text { Moore et al. } \\
\quad(2007)^{(11)} \\
\text { Canada }\end{array}$ & $\begin{array}{l}162 \text { male children aged } \\
9-12 \text { and } 14-16 \text { years } \\
\text { Children } \\
\text { Adolescents }\end{array}$ & $\begin{array}{l}\text { Rapid assessment method (RAM) } \\
\text { FFQ } \\
\text { thirty-two food items; intakes } \\
\text { refer to a 'typical day'. Included } \\
\text { supplements. Visual aids to } \\
\text { approximate portion size. } \\
\text { Interviewer-administered }\end{array}$ & $\begin{array}{l}\text { Single } 24 \mathrm{~h} \text { dietary recalls } \\
\text { Visual aids } \\
\text { Interviewer-administered } \\
\text { during same interview as } \\
\text { RAM }\end{array}$ & $\mathrm{Ca}$ & $\begin{array}{l}\text { Correlations between questionnaire } \\
\text { and recalls were significant but } \\
\text { moderate, though the RAM } \\
\text { overestimates daily } \mathrm{Ca} \text { intake as } \\
\text { compared with the } 24 \mathrm{~h} \text { recall } \\
\text { method in both children and } \\
\text { adolescent males. }\end{array}$ \\
\hline $\begin{array}{l}\text { Lillegaard et al. } \\
\quad(2007)^{(12)} \\
\quad \text { Norway }\end{array}$ & $\begin{array}{l}\text { Forty-five girls and fifty-five } \\
\text { boys } 9 \text { years old } \\
\text { Children }\end{array}$ & $\begin{array}{l}4 \mathrm{~d} \text { pre-coded food diaries (PFD) } \\
277 \text { food items } \\
\text { Portion size in household } \\
\text { measures or using colour photo- } \\
\text { graphs }\end{array}$ & $\begin{array}{l}4 \mathrm{~d} \text { weighed dietary records } \\
\text { (same days of week as } \\
\text { PFD, } 1 \text { week later) } \\
\text { Records included sup- } \\
\text { plements }\end{array}$ & $\begin{array}{l}\text { Retinol, vitamins } \mathrm{D}, \mathrm{C} \text {, } \\
\text { thiamin, riboflavin, } \\
\mathrm{Ca}, \mathrm{Fe}\end{array}$ & $\begin{array}{l}\text { The PFD method is promising as a } \\
\text { tool for assessing food intake in } \\
\text { large surveys among children. }\end{array}$ \\
\hline $\begin{array}{l}\text { Magkos et al. } \\
(2006)^{(13)} \\
\text { Greece }^{\text {G }}\end{array}$ & $\begin{array}{l}351 \text { children ( } 189 \text { girls and } \\
162 \text { boys) } \\
\text { Mean age } 11.9 \text { years } \\
\text { Children }\end{array}$ & $\begin{array}{l}\text { 30-item FFQ } \\
\text { Past } 12 \text { months intake Standard } \\
\text { reference portions } \\
\text { Self-administered }\end{array}$ & $\begin{array}{l}\text { Single Multipass 24HR } \\
\text { Interviewer-administered } \\
\text { subsequent to FFQ. Stan- } \\
\text { dard household measures } \\
\text { (cups, tablespoons, etc) \& } \\
\text { Picture food models }\end{array}$ & $\mathrm{Ca}$ & $\begin{array}{l}\text { The FFQ can be used to discrimi- } \\
\text { nate high } v \text {. low Ca intakes, but } \\
\text { results do not support its use in } \\
\text { epidemiological studies for the } \\
\text { quantitative assessment of } \\
\text { individual Ca intake. }\end{array}$ \\
\hline $\begin{array}{l}\text { Harnack et al. } \\
\qquad(2006)^{(14)} \text { USA }\end{array}$ & $\begin{array}{l}248 \text { children } \\
\text { Aged } 11-14 \text { years } \\
\text { Children } \\
\text { Adolescents }\end{array}$ & $\begin{array}{l}\text { 10-item FFQ Past month's intake. } \\
\text { Portion size alternatives included. } \\
\text { Frequency categories up to } 3 / \mathrm{d} \text {. } \\
\text { Self-administered. }\end{array}$ & $\begin{array}{l}\text { Three } 24 \text { hour dietary recalls } \\
\text { by phone subsequent to } \\
\text { FFQ. } \\
\text { 2D food model visual aids } \\
\text { mailed }\end{array}$ & $\mathrm{Ca}$ & $\begin{array}{l}\text { The Ca FFQ evaluated in this study } \\
\text { may be useful where a brief } \\
\text { instrument is needed, with a } \\
\text { moderate association with } \\
\text { estimates from dietary recalls. }\end{array}$ \\
\hline $\begin{array}{l}\text { Huybrechts et al. } \\
(2006)^{(15)} \\
\text { Belgium }\end{array}$ & $\begin{array}{l}509 \text { preschool children } \\
(2 \cdot 5-6.5 \text { years }) \\
\text { Preschool children }\end{array}$ & $\begin{array}{l}\text { FFQ } \\
\text { Past } 12 \text { months intake six } \\
\text { frequency categories up to one }\end{array}$ & $\begin{array}{l}3 \mathrm{~d} \text { estimated dietary records } \\
\text { (three consecutive days) }\end{array}$ & $\mathrm{Ca}$ & $\begin{array}{l}\text { This FFQ tended to underestimate } \\
\text { preschool children's Ca intake } \\
\text { but had a fairly good ability to }\end{array}$ \\
\hline
\end{tabular}




\begin{tabular}{|c|c|c|c|c|c|}
\hline $\begin{array}{l}\text { Author/year } \\
\text { publication } \\
\text { and country }\end{array}$ & Participants/age group & Dietary method & Reference method & Micronutrient & Conclusions \\
\hline & & $\begin{array}{l}\text { daily intake. Forty-seven food } \\
\text { items. Completed by the child's } \\
\text { parent or guardian. Portion } \\
\text { size alternatives included. } \\
\text { Self-administered. }\end{array}$ & & & classify subjects. \\
\hline $\begin{array}{l}\text { Bertoli et al. } \\
\qquad(2005)^{(16)} \text { Italy }\end{array}$ & $\begin{array}{l}\text { Eighteen children aged } \\
6-10 \text { years nineteen } \\
\text { adolescents aged } 16-20 \\
\text { years } \\
\text { Children } \\
\text { Adolescents }\end{array}$ & $\begin{array}{l}\text { FFQ } \\
136 \text { food items (period of intake not } \\
\text { reported). Included supplements. } \\
\text { Portion size } \\
\text { estimated using colour } \\
\text { photographs (small, medium, } \\
\text { large). Seven frequency } \\
\text { categories. Interviewer- } \\
\text { administered; children aided by } \\
\text { parents as needed. }\end{array}$ & $7 \mathrm{~d}$ weighed dietary records & $\mathrm{Ca}$ & $\begin{array}{l}\text { The FFQ has proved adequate to } \\
\text { rank a paediatric population in } \\
\text { terms of } \mathrm{Ca} \text { intake. }\end{array}$ \\
\hline $\begin{array}{l}\text { Williams \& Innis } \\
(2005)^{(17)} \\
\text { Canada }\end{array}$ & $\begin{array}{l}148 \text { infants aged } 8-26 \\
\text { months } \\
\text { Infants }\end{array}$ & $\begin{array}{l}\text { FFQ } \\
191 \text { foods } \\
\text { Previous } 2 \text { weeks intakelncluded } \\
\text { supplements } \\
\text { Interviewer-administered }\end{array}$ & $\begin{array}{l}\text { (i) } 3 \text { d estimated dietary } \\
\text { records } \\
\text { ( } 3 \text { consecutive weekdays } \\
\text { and } 1 \text { weekend day). } \\
\text { Household } \\
\text { measuring utensils } \\
\text { provided Completed } \\
1 \text { week before food } \\
\text { records } \\
\text { (ii) Biomarkers: Serum } \\
\text { markers of Fe status } \\
\text { (ferritin, haemoglobin) }\end{array}$ & $\mathrm{Fe}$, vitamin $\mathrm{C}, \mathrm{Ca}$ & $\begin{array}{l}\text { FFQ need further development } \\
\text { before they can be used to } \\
\text { advance assessment of Fe intake } \\
\text { and status in infants (poor } \\
\text { correlations with biomarkers). }\end{array}$ \\
\hline $\begin{array}{l}\text { Andersen et al. } \\
(2004)^{(18,19)} \\
\text { Norway }\end{array}$ & $\begin{array}{l}187 \\
2 \text {-year-old children } \\
\text { Preschool children }\end{array}$ & $\begin{array}{l}\text { Semi-quantitative FFQ (SFFQ) } \\
\text { Previous } 14 \text { days intake. } 125 \text { food } \\
\text { items Included supplements. } \\
\text { Portion size estimated using food } \\
\text { photographs or household } \\
\text { measures Self-administered }\end{array}$ & $\begin{array}{l}7 \mathrm{~d} \text { weighed dietary records } \\
\text { ( } 7 \text { consecutive days) For } \\
\text { breast-fed infants: how } \\
\text { often breast milk } \\
\text { was given. }\end{array}$ & $\begin{array}{l}\text { Vitamins } \mathrm{A}, \mathrm{D}, \mathrm{E}, \mathrm{C} \text {, thiamin, } \\
\quad \text { riboflavin, } \mathrm{Ca}, \mathrm{Fe}\end{array}$ & $\begin{array}{l}\text { The SFFQ may be a valuable tool } \\
\text { for measuring average intakes of } \\
\text { energy, macronutrients and } \\
\text { several food items for a } \\
\text { 2-year-old population in Norway. }\end{array}$ \\
\hline $\begin{array}{l}\text { Slater et al. } \\
\qquad(2003)^{(20)} \text { Brazil }\end{array}$ & $\begin{array}{l}\text { Seventy-nine adolescents } \\
\text { Aged } 14-18 \text { years } \\
\text { Adolescents }\end{array}$ & $\begin{array}{l}\text { FFQ for adolescents (AFFQ) } \\
\text { Seventy-six food items. Past } \\
6 \text { months intake seven frequency } \\
\text { category. Single reference portion } \\
\text { sizes listed. Use of interviewers } \\
\text { not reported }\end{array}$ & $\begin{array}{l}\text { Three } 24 \mathrm{~h} \text { dietary recalls } \\
\text { At intervals of } 45 \text { days } \\
\text { before FFQ administration }\end{array}$ & Retinol, vitamin $\mathrm{C}, \mathrm{Ca}, \mathrm{Fe}$ & $\begin{array}{l}\text { The FFQ provides a reliable scale } \\
\text { for categorising individuals by } \\
\text { level of past intake of most } \\
\text { nutrients, excluding retinol } \\
\text { and Fe. }\end{array}$ \\
\hline $\begin{array}{l}\text { Marshall et al. } \\
(2003)^{(21)} \text { USA }\end{array}$ & $\begin{array}{l}240 \text { children } \\
6 \& 12 \text { months } 3 \text { \& } 5 \text { years } \\
\text { Infants } \\
\text { Preschool children }\end{array}$ & $\begin{array}{l}\text { Quantitative beverage FFQ; seven } \\
\text { food items or groups described. } \\
\text { Intakes in preceding week: at } \\
6 \text { and } 12 \text { months parents } \\
\text { reported average daily intake; at } \\
3 \text { and } 5 \text { years average number of } \\
\text { servings per week and usual } \\
\text { serving size for each beverage } \\
\text { Self-administered. }\end{array}$ & $\begin{array}{l}3 \mathrm{~d} \text { weighed dietary records } \\
\text { ( } 2 \text { weekdays and } \\
1 \text { weekend day) Intakes } \\
\text { from food and beverages } \\
\text { (reported here) as well as } \\
\text { beverages only (similar } \\
\text { results) analysed }\end{array}$ & Ca, vitamin D & $\begin{array}{l}\text { A quantitative beverage frequency } \\
\text { questionnaire can provide a } \\
\text { relative estimate of beverage, } \mathrm{Ca} \\
\text { and vitamin } \mathrm{D} \text { intakes }\end{array}$ \\
\hline
\end{tabular}


Table 2. Continued

\begin{tabular}{|c|c|c|c|c|c|}
\hline $\begin{array}{l}\text { Author/year } \\
\text { publication } \\
\text { and country }\end{array}$ & Participants/age group & Dietary method & Reference method & Micronutrient & Conclusions \\
\hline $\begin{array}{l}\text { Andersen et al. } \\
(2003)^{(2)} \\
\text { Norway }\end{array}$ & $\begin{array}{l}\text { Sixty-four infants } \\
12 \text {-month-old } \\
\text { Infants }\end{array}$ & $\begin{array}{l}\text { Semi-quantitative FFQ; Forty-four } \\
\text { questions about } 140 \text { food items } \\
\text { and supplements Parents asked } \\
\text { to keep in mind past } 14 \text { days } \\
\text { intake. Food photographs or } \\
\text { household measures to estimate } \\
\text { portions. For breast-fed infants: } \\
\text { how often breast milk was given } \\
\text { per } 24 \mathrm{~h} \text { Self-administered }\end{array}$ & $\begin{array}{l}7 \mathrm{~d} \text { weighed dietary records } \\
\text { (four consecutive days, } \\
\text { 1-week interval, and three } \\
\text { consecutive days) } \\
\text { For breast-fed infants: } \\
\text { how often breast milk was } \\
\text { given. }\end{array}$ & $\begin{array}{l}\text { Vitamins } \mathrm{A}, \mathrm{D}, \mathrm{E}, \mathrm{C} \text {, thiamin, } \\
\text { riboflavin, } \mathrm{Ca}, \mathrm{Fe}\end{array}$ & $\begin{array}{l}\text { The capability of the questionnaire } \\
\text { to rank infants according to intake } \\
\text { of nutrients and food items was } \\
\text { moderate. }\end{array}$ \\
\hline $\begin{array}{l}\text { Parrish et al. } \\
(2003)^{(22)} \text { USA }\end{array}$ & $\begin{array}{l}\text { Sixty-eight children aged } \\
1-3 \text { years (biomarker } \\
n 38 \text { ) } \\
\text { Infants } \\
\text { Preschool children }\end{array}$ & $\begin{array}{l}\text { (i) FFQ; Past } 12 \text { months intake. } \\
111 \text { food items. Completed by } \\
\text { the child's parent or guardian at } \\
\text { end of study period (self-adminis- } \\
\text { tered). Standard reference } \\
\text { portions. } \\
\text { (ii) Four } 24 \mathrm{~h} \text { dietary recalls Inter- } \\
\text { viewer-administered to parent } \\
\text { and alternate caregiver every } 3 \\
\text { months before FFQ }\end{array}$ & $\begin{array}{l}\text { Biomarkers } \\
\text { Plasma levels of vitamins } \\
C, D \text {, retinol, } \\
\beta \text {-carotene and } \\
\alpha \text {-tocopherol (vitamin E) }\end{array}$ & $\begin{array}{l}\text { Vitamins D, C, retinol, } \\
\beta \text {-carotene, } \alpha \text {-tocopherol } \\
\text { (vitamin E) }\end{array}$ & $\begin{array}{l}\text { The FFQ shows mostly good } \\
\text { agreements with multiple } 24 \mathrm{~h} \\
\text { recalls and some biomarkers in } \\
\text { preschool children. }\end{array}$ \\
\hline $\begin{array}{l}\text { Lietz et al. } \\
\qquad(2002)^{(23)} \text { UK }\end{array}$ & $\begin{array}{l}\text { Fifty children aged } \\
11-13 \text { years } \\
\text { (biomarker } n 27 \text { ) } \\
\text { Children }\end{array}$ & $\begin{array}{l}\text { (i) EPIC FFQ; } 130 \text { items. Inter- } \\
\text { viewer-administered. Past } \\
12 \text { months intake; nine frequency } \\
\text { categories. Standard reference } \\
\text { portions used. } \\
\text { (ii) } 7 \mathrm{~d} \text { weighed dietary records } \\
\text { subsequent to FFQ. Food photo- } \\
\text { graphs to } \\
\text { estimate portions when weighing } \\
\text { not possible. }\end{array}$ & $\begin{array}{l}\text { (i) } 7 \mathrm{~d} \text { weighed dietary } \\
\text { records } \\
\text { After FFQ. Food photo- } \\
\text { graphs } \\
\text { (ii) Biomarkers: } 24 \mathrm{~h} \\
\text { urine K }\end{array}$ & $\mathrm{Ca}, \mathrm{Na}, \mathrm{K}$ & $\begin{array}{l}\text { The EPIC FFQ is not an } \\
\text { appropriate method for estimating } \\
\text { absolute intakes in adolescent } \\
\text { population. However, it seems } \\
\text { adequate to classify low, medium } \\
\text { and high consumers and might } \\
\text { therefore be used to identify } \\
\text { population groups at risk. }\end{array}$ \\
\hline $\begin{array}{l}\text { Blum et al. } \\
\qquad(1999)^{(24)} \text { USA }\end{array}$ & $\begin{array}{l}233 \text { children } \\
\text { Aged } 1-5 \text { years } \\
\text { Infants } \\
\text { Preschool children }\end{array}$ & $\begin{array}{l}\text { Harvard Service FFQ (HFFQ) } \\
\text { eighty-four food items + nineteen } \\
\text { items on supplements, food } \\
\text { habits. Past 4-week intakes. Age- } \\
\text { appropriate portions applied (not } \\
\text { shown on FFQ). Completed by } \\
\text { the child's parent or guardian } \\
\text { (self-administered). Mean of two } \\
\text { FFQ } 1 \text { month apart. }\end{array}$ & $\begin{array}{l}\text { Three } 24 \mathrm{~h} \text { dietary recalls at } \\
7-10 \mathrm{~d} \text { intervals between } \\
1 \text { st and } 2 \text { nd FFQ. } \\
\text { Two recalls on weekdays } \\
\text { and one recall on } \\
\text { weekend. } \\
\text { Interviewer-administered } \\
\text { by telephone or in person. }\end{array}$ & $\begin{array}{l}\text { Vitamins } \mathrm{A}, \mathrm{E}, \mathrm{C}, \mathrm{B}_{6}, \mathrm{~B}_{12} \text {, } \\
\text { thiamin, riboflavin, niacin, } \\
\text { folate, } \mathrm{Ca}, \mathrm{Zn}, \mathrm{Mg}, \mathrm{Fe}\end{array}$ & $\begin{array}{l}\text { The HFFQ is a simple self- } \\
\text { administered questionnaire } \\
\text { completed by the child's parent } \\
\text { or guardian and is useful in } \\
\text { assessing the diets of Native } \\
\text { American and Caucasian } \\
\text { children. }\end{array}$ \\
\hline $\begin{array}{l}\text { Field et al. } \\
\qquad(1999)^{(25)} \text { USA }\end{array}$ & $\begin{array}{l}109 \text { students from fourth to } \\
\text { seventh grade } \\
\text { Children }\end{array}$ & $\begin{array}{l}\text { FFQ } \\
\text { Past } 12 \text { months intake } \\
\text { Self-administered (read aloud by } \\
\text { teachers) } \\
\text { ninety-seven food items, standard } \\
\text { reference } \\
\text { portions used. }\end{array}$ & $\begin{array}{l}\text { Four } 24 \mathrm{~h} \text { dietary recalls } \\
\text { (collected on non- } \\
\text { consecutive days approxi- } \\
\text { mately } 3 \text { months apart) } \\
\text { Interviewer-administered } \\
\text { before FFQ Portion size in } \\
\text { household measures or } \\
\text { using colour photographs }\end{array}$ & Vitamin C, P, Ca, Fe & $\begin{array}{l}\text { Sixth and seventh grade students } \\
\text { demonstrated the ability to } \\
\text { provide valid estimates of intake, } \\
\text { but children in the fourth and fifth } \\
\text { grades experienced some } \\
\text { difficulty in completing the FFQ. }\end{array}$ \\
\hline $\begin{array}{l}\text { Taylor \& Goulding } \\
(1998)^{(26)} \\
\text { New Zealand }\end{array}$ & $\begin{array}{l}\text { Forty-one girls and } \\
\text { twenty-six boys } \\
\text { aged } 3-6 \text { years }\end{array}$ & $\begin{array}{l}\text { FFQ } \\
\text { Past } 12 \text { months intake } \\
\text { thirty-five food and }\end{array}$ & $\begin{array}{l}4 \mathrm{~d} \text { estimated dietary } \\
\text { records }\end{array}$ & $\mathrm{Ca}$ & $\begin{array}{l}\text { The short Ca FFQ tended to } \\
\text { overestimate actual Ca intakes in } \\
\text { young children. However, the }\end{array}$ \\
\hline
\end{tabular}




\begin{tabular}{|c|c|}
\hline $\begin{array}{l}\text { Author/year } \\
\text { publication } \\
\text { and country }\end{array}$ & Participants/age group \\
\hline & $\begin{array}{l}\text { Preschool children } \\
\text { Children }\end{array}$ \\
\hline $\begin{array}{l}\text { Mølgaard et al. } \\
(1998)^{(27)} \\
\text { Denmark }\end{array}$ & $\begin{array}{l}\text { Twenty-three children } \\
\text { aged } 13-14 \text { years } \\
\text { Adolescents }\end{array}$ \\
\hline $\begin{array}{l}\text { Green et al. } \\
\quad(1998)^{(28)} \\
\text { Canada }\end{array}$ & $\begin{array}{l}105 \text { adolescent females } \\
\text { aged } 16-19 \text { years } \\
\text { Adolescents }\end{array}$ \\
\hline $\begin{array}{l}\text { Rockett et al. } \\
\qquad(1997)^{(29)} \text { USA }\end{array}$ & $\begin{array}{l}261 \text { children and } \\
\text { adolescents (aged 9-18) } \\
\text { Children } \\
\text { Adolescents }\end{array}$ \\
\hline $\begin{array}{l}\text { Bellù et al. } \\
\qquad(1996)^{(30)} \text { Italy }\end{array}$ & $\begin{array}{l}333 \text { children } \\
\text { ( } 158 \text { girls, } 165 \text { boys }) \text { aged } \\
8-10 \text { years } \\
\text { Children }\end{array}$ \\
\hline $\begin{array}{l}\text { Arnold et al. } \\
\quad(1995)^{(31)} \\
\text { Canada }\end{array}$ & $\begin{array}{l}707 \text { girls } \\
\quad \text { aged } 7-12 \text { years } \\
\text { Children }\end{array}$ \\
\hline $\begin{array}{l}\text { Bellù et al. } \\
\qquad(1995)^{(32)} \text { Italy }\end{array}$ & $\begin{array}{l}\text { Eighty-eight children } \\
\text { (forty-nine girls, thiry-nine } \\
\text { boys) aged 9-12 years } \\
\text { Children }\end{array}$ \\
\hline $\begin{array}{l}\text { Andersen et al. } \\
(1995)^{(33)} \\
\text { Norway }\end{array}$ & $\begin{array}{l}\text { Forty-nine adolescents } \\
\text { (17-18 years) } \\
\text { Adolescents }\end{array}$ \\
\hline
\end{tabular}

Dietary method

drink items

Self-administered together with their parents. Past 1 month's intake eighty-eight food items. Exact frequencies used Portion size in household measures

(i) FFQ Self-administered

116 food items. Included sup-

plements Past 12 months intake. Standard

reference portion sizes in

household measures

(ii) $3 \mathrm{~d}$ weighed records

(weekdays, weekends)

Youth/Adolescent Questionnaire

(YAQ)

Self-administered

131 items; standard reference portions. Past 12 months intake. Mean of two questionnaires administered approximately 1.5 years apart

FFQ

116 food items

\section{FFQ}

160 food items. Adapted from Block FFQ (intakes in past year) Standard reference portions. Exact frequencies Interviewers reviewed reponses.

FFQ

116 food items. Reference portion sizes Self-administered

FFQ

190 food items. Portion size in household measures, variable portion sizes included. Meal-based reporting Exact frequencies. Past 12 months intake

Reference method

Micronutrient

Conclusions

FFQ demonstrated good ability to classify subjects into extremes of Ca intake.

$3 \mathrm{~d}$ weighed dietary $\quad \mathrm{Ca}, \mathrm{P}$

records

Completed after FFQ 2

weekdays,

1 weekend day

Biomarkers

Serum folate and serum vitamin $\mathrm{B}_{12}$

Folate, vitamin $B_{12}$

Three $24 \mathrm{~h}$ dietary recalls a approximately 5-month intervals, with detailed

probing. Administered

between 1st and 2nd

FFQ.

$24 \mathrm{~h}$ dietary recalls Interviewer-administered (children's mothers)

3D-food models

Two $7 \mathrm{~d}$ estimated dietary records

$7 \mathrm{~d}$ estimated dietary records

3D-food models Completed after FFQ

$7 \mathrm{~d}$ weighed dietary records 4 consecutive days, 1 week interval and three consecutive days (five week days and two weekend days) Completed 2-3 months after FFQ
Vitamins $A, E, C, B_{6}, B_{12}$, retinol, thiamin, riboflavin, niacin, folate, $\mathrm{Ca}, \mathrm{P}, \mathrm{Mg}$,

$\mathrm{Fe}, \mathrm{Zn}, \mathrm{Cu}, \mathrm{K}, \mathrm{Na}$

$\mathrm{Ca}, \mathrm{Fe}$

Vitamins $\mathrm{A}, \mathrm{C}$, riboflavin, thiamin, retinol, $\beta$-carotene

Vitamins $A, C, E, B_{6}$ riboflavin, thiamin, $\mathrm{Ca}, \mathrm{Fe}$

Retinol, vitamins D, C, thiamin, riboflavin, $\mathrm{Ca}, \mathrm{Fe}$ $\mathrm{Mg}$
The FFQ is able to rank children according to their intake of $\mathrm{Ca}$ and $\mathrm{P}$.

The FFQ and $3 \mathrm{~d}-\mathrm{WDR}$ are valid measure of assessing folate intakes in young women compared with biomarkers. Both appear useful in determining vitamin $B_{12}$ intake, but only when supplement users are included.

A simple self-administered questionnaire completed by older children and adolescents can provide nutritional information about this age group.

Average estimates with the FFQ agree with findings obtained by $24 \mathrm{~h}$ recalls for most nutrients. The FFQ we designed is appropriate in the northern Italian paediatric population.

$70 \%$ of subjects were categorised in the highest or lowest quartile by both FFQ and $7 \mathrm{~d}$ EDR for energy and several nutrients.

Intake estimates for most nutrients were comparable, but there were significant differences in estimates for several nutrients. Use of nutrient density reduced misclassification.

The questionnaire is able to rank subjects according to the tested nutrients $(0-8 \%$ classified in extreme quartiles), except vitamin D (12\% in extreme quartile). 


\begin{tabular}{|c|c|c|c|c|c|}
\hline $\begin{array}{l}\text { Author/year } \\
\text { publication } \\
\text { and country }\end{array}$ & Participants/age group & Dietary method & Reference method & Micronutrient & Conclusions \\
\hline $\begin{array}{l}\text { lannotti et al. } \\
(1994)^{(34)} \text { USA }\end{array}$ & $\begin{array}{l}\text { Seventeen preschool } \\
\text { children } 2-4 \text { years } \\
\text { Preschool children }\end{array}$ & $\begin{array}{l}\text { Willett FFQ } \\
\text { Previous } 7 \text { days intake } \\
\text { Self-administered } \\
\text { six frequency category }\end{array}$ & $\begin{array}{l}\text { Three } 24 \mathrm{~h} \text { dietary recalls } \\
\text { before FFQ. }\end{array}$ & $\mathrm{Na}$ & $\begin{array}{l}\text { The questionnaire did not provide } \\
\text { an accurate assessment of } \\
\text { measured intake and could not } \\
\text { replace the dietary recall. }\end{array}$ \\
\hline $\begin{array}{l}\text { Byers et al. } \\
\qquad(1993)^{(35)} \text { USA }\end{array}$ & $\begin{array}{l}\text { Ninety-seven children aged } \\
6-10 \text { years } \\
\text { Children }\end{array}$ & $\begin{array}{l}\text { FFQ } \\
\text { Past } 3 \text { months intake } \\
\text { Completed by the child's parent } \\
\text { Interviewer-administered. } 111 \\
\text { food items Frequency categories } \\
\text { based on Willett }\end{array}$ & $\begin{array}{l}\text { Biomarkers } \\
\text { Serum levels of vitamins } \\
\mathrm{C}, \mathrm{A} \text { and } \mathrm{E}\end{array}$ & Vitamins C, A, E & $\begin{array}{l}\text { Children in the highest } v \text {. lowest } \\
\text { fruit and vegetable intake } \\
\text { quartiles had } 31 \% \text { higher serum } \\
\text { vitamin C. Parental reports of } \\
\text { young children's diet using FFQ } \\
\text { methods are accurate enough to } \\
\text { be useful in nutritional screening. }\end{array}$ \\
\hline $\begin{array}{l}\text { Lytle et al. } \\
\qquad(1993)^{(36)} \text { USA }\end{array}$ & $\begin{array}{l}\text { Forty-nine children aged } \\
8 \text { years old } \\
\text { Children }\end{array}$ & $\begin{array}{l}24 \mathrm{~h} \text { dietary recalls } \\
\text { administered to the child, aided } \\
\text { by a previous day food record } \\
\text { (listing) Interviewer-administered. } \\
\text { 3D food models and utensils. }\end{array}$ & $\begin{array}{l}\text { Observed intakes } \\
\text { (parents and trained staff) }\end{array}$ & $\mathrm{Na}$ & $\begin{array}{l}\text { The } 24 \mathrm{~h} \text { recall assisted by food } \\
\text { records is a valid method for } \\
\text { assessing the dietary intake of } \\
\text { children as young as } 8 \text { years old. }\end{array}$ \\
\hline $\begin{array}{l}\text { Stein et al. } \\
\qquad(1992)^{(37)} \text { USA }\end{array}$ & $\begin{array}{l}224 \text { preschool children } \\
\text { (baseline aged } \\
44-60 \text { months) } \\
\text { Preschool children }\end{array}$ & $\begin{array}{l}\text { Willett semi-quantitative FFQ } \\
\text { Past } 6 \text { months intake. Completed } \\
\text { by the child's parent. Interviewer- } \\
\text { administered. Mean of two FFQ } \\
\text { completed at } 6 \text { months intervals. } \\
\text { Reference portion sizes adapted } \\
\text { for children. }\end{array}$ & $\begin{array}{l}\text { Four } 24 \mathrm{~h} \text { dietary recalls } \\
\text { Interviewer-administered } \\
\text { before each FFQ 3D-food } \\
\text { models }\end{array}$ & $\mathrm{Na}, \mathrm{K}, \mathrm{Ca}$ & $\begin{array}{l}\text { Agreement between the two } \\
\text { methods in assigning individuals } \\
\text { to energy-adjusted quintiles was } \\
\text { moderate, as were correlations } \\
\text { between energy-adjusted intakes. }\end{array}$ \\
\hline $\begin{array}{l}\text { Jenner et al. } \\
(1989)^{(38)} \\
\text { Australia }\end{array}$ & $\begin{array}{l}225 \text { children Aged } \\
11-12 \text { years } \\
\text { Children }\end{array}$ & $\begin{array}{l}\text { FFQ } \\
175 \text { food items. Previous } 7 \text { days } \\
\text { intake. Meal-based } \\
\text { reporting. six frequency } \\
\text { categories. No portions on FFQ } \\
\text { (average portions from records } \\
\text { applied). Administered separately } \\
\text { to (i) children and (ii) parents. A } \\
\text { subset of the first } 1-3 \text { diet } \\
\text { records were also used as an } \\
\text { alternative intake estimate. }\end{array}$ & $\begin{array}{l}\text { Fourteen } 24 \mathrm{~h} \text { dietary } \\
\text { records Completed over } \\
\text { 3-month period after FFQ }\end{array}$ & $\mathrm{K}, \mathrm{Ca}$ & $\begin{array}{l}\text { Poor agreement between the diet } \\
\text { records and the FFQ completed } \\
\text { either by children or parents. In } \\
\text { contrast, agreement between the } \\
\text { reference method and } 1-3 \text { diet } \\
\text { records in the series was } \\
\text { relatively good. }\end{array}$ \\
\hline $\begin{array}{l}\text { Räsänen L. } \\
(1979)^{(39)} \\
\text { Finland }\end{array}$ & $\begin{array}{l}741 \text { children aged } \\
5-13 \text { years } \\
\text { Preschool children } \\
\text { Children } \\
\text { Adolescents }\end{array}$ & $\begin{array}{l}\text { Single } 24 \mathrm{~h} \text { dietary recalls } \\
\text { Interviewer-administered Com- } \\
\text { pleted by the child's mothers } \\
\text { Included supplements Portion } \\
\text { size in household measures }\end{array}$ & $\begin{array}{l}\text { Diet history } \\
\text { Past } 12 \text { months intake } \\
\text { Interviewer-administered }\end{array}$ & $\begin{array}{l}\text { Ca, Fe, vitamins } A, C \text {, } \\
\text { thiamin, riboflavin, niacin }\end{array}$ & $\begin{array}{l}\text { Neither of the methods can be } \\
\text { considered suitable for the } \\
\text { measurement of an individual } \\
\text { child's dietary intake. }\end{array}$ \\
\hline
\end{tabular}

$24 \mathrm{HR}, 24 \mathrm{~h}$ diet recalls; EPIC, european prospective investigation of cancer; WDR, weighed dietary record; EDR, estimated dietary record. 


\begin{tabular}{|c|c|c|c|c|}
\hline \multirow{2}{*}{$\begin{array}{l}\text { Author/year } \\
\text { publication/country/ } \\
\text { (quality index) } \\
\text { Vitamin A }\end{array}$} & \multirow[t]{2}{*}{ n/age group } & \multirow[t]{2}{*}{$\begin{array}{l}\text { Methods/ } \\
\text { no FFQ items, } \\
\text { portion sizes }\end{array}$} & \multicolumn{2}{|c|}{ Correlation between methods/other statistics } \\
\hline & & & & \\
\hline $\begin{array}{l}\text { Andersen et al. } \\
(2003)^{(2)} \text { Norway } \\
(4 \cdot 5)\end{array}$ & $\begin{array}{l}n 64 \\
12 \text { months } \\
\text { Infants }\end{array}$ & $\begin{array}{l}\text { FFQ } v .7 \mathrm{~d} \text { WDR } \\
140 \text { items. HH } \\
\text { measures or food } \\
\text { photographs }\end{array}$ & $\begin{array}{l}\text { Absolute intake } \\
\text { CC } 0.34\end{array}$ & $\begin{array}{l}\text { Median }\left(\mathrm{P}_{25}-\mathrm{P}_{75}\right)(\mu \mathrm{g}) \\
\text { FFQ } 1358(1008-3029) \\
\text { WDR } 681(490-834)^{\star \star \star}\end{array}$ \\
\hline $\begin{array}{l}\text { Blum et al. } \\
(1999)^{(24)} \text { USA (2.5) }\end{array}$ & $\begin{array}{l}n 233 \\
1-5 \text { years } \\
\text { Infants } \\
\text { Preschool children }\end{array}$ & $\begin{array}{l}\mathrm{FFQ} v \text {. three } 24 \mathrm{HR} \\
\text { eighty-four items. }\end{array}$ & CC 0.49 & $\begin{array}{l}\text { Mean (sD) (RE) } \\
24 \text { HR } 1035(569) \\
\text { FFQ } 1176(449)\end{array}$ \\
\hline $\begin{array}{l}\text { Andersen et al. } \\
(2004)^{(18,19)} \\
\text { Norway (5) }\end{array}$ & $\begin{array}{l}n 187 \\
2 \text { years old } \\
\text { Preschool children }\end{array}$ & $\begin{array}{l}\text { FFQ v. } 7 \mathrm{~d} \text { WDR } \\
125 \text { items. HH } \\
\text { measures or food } \\
\text { photographs }\end{array}$ & $\begin{array}{l}\text { Absolute intake } \\
\text { CC } 0.59^{\star \star \star}\end{array}$ & $\begin{array}{l}\text { Median }\left(P_{25}-P_{75}\right)(\mu \mathrm{g}) \\
\text { FFQ } 1586(1130-2230) \\
\text { WDR } 694(473-1027)^{\star \star \star}\end{array}$ \\
\hline $\begin{array}{l}\text { Räsänen L. } \\
(1979)^{(39)} \text { Finland } \\
(5)\end{array}$ & $\begin{array}{l}n 741 \\
5-13 \text { years } \\
\text { Preschool children } \\
\text { Children } \\
\text { Adolescents }\end{array}$ & $24 \mathrm{HR} v . \mathrm{DH}$ & $\mathrm{CC} 0.20$ & Mean DH: 1650; 24 HR: 1061 \\
\hline $\begin{array}{l}\text { Byers et al. } \\
(1993)^{(35)} \text { USA (2.5) }\end{array}$ & $\begin{array}{l}n 97 \\
6-10 \text { years } \\
\text { Children }\end{array}$ & $\begin{array}{l}\mathrm{FFQ} v . \mathrm{BM} \\
111 \text { items }\end{array}$ & $\begin{array}{l}\text { Crude } 0.14 \\
\text { Adjusted } 0.17\end{array}$ & \\
\hline $\begin{array}{l}\text { Arnold et al. } \\
(1995)^{(31)} \text { Canada } \\
(3 \cdot 5)\end{array}$ & $\begin{array}{l}n 707 \\
7-12 \text { years } \\
\text { Children }\end{array}$ & $\begin{array}{l}\text { FFQ } v .14 \text { d EDR } \\
160 \text { items. Standard } \\
\text { reference portions }\end{array}$ & Pearson CC $0.49 \dagger$ & \\
\hline $\begin{array}{l}\text { Bellù et al. } \\
\text { (1995) }\end{array}$ & $\begin{array}{l}n 88 \\
9-12 \text { years } \\
\text { Children }\end{array}$ & $\begin{array}{l}\text { FFQ } v .7 \mathrm{~d} \text { EDR } \\
116 \text { items. Reference } \\
\text { portion sizes }\end{array}$ & $\begin{array}{l}\text { Raw nutrient } 0.06 \\
\text { Nutrient density } 0.13\end{array}$ & $\begin{array}{l}\text { Mean (SD) } \\
\text { EDR } 419(740) \\
\text { FFQ } 371(157)\end{array}$ \\
\hline $\begin{array}{l}\text { Rockett et al. } \\
(1997)^{(29)} \text { USA (3.5) }\end{array}$ & $\begin{array}{l}n 261 \\
9-18 \text { years } \\
\text { Children } \\
\text { Adolescents }\end{array}$ & $\begin{array}{l}\text { YAQ v. three } 24 \mathrm{HR} \\
131 \text { items. Standard } \\
\text { reference portions }\end{array}$ & $\begin{array}{l}9-13 \text { years } C C 0.39 \\
14-18 \text { years } C C 0.37\end{array}$ & $\begin{array}{l}\text { Mean (SD) (IU) } \\
24 \text { HR } 6755(5023) \\
\text { YAQ } 9228(4317)\end{array}$ \\
\hline \multicolumn{5}{|l|}{ Retinol } \\
\hline $\begin{array}{l}\text { Marriott et al. } \\
(2008)^{(8)} \text { UK (3.5) }\end{array}$ & $\begin{array}{l}n 50 \\
6 \text { months } \\
\text { Infants }\end{array}$ & $\begin{array}{l}\text { FFQ } v .4 \mathrm{~d} \text { WDR } \\
\text { thirty-four items. HH } \\
\text { measures }\end{array}$ & $\begin{array}{l}\text { Energy adjusted } \\
\text { CC } 0.76\end{array}$ & $\begin{array}{l}\text { Median }(\mu \mathrm{g}) \\
\text { FFQ } 597 \\
\text { WDR } 531\end{array}$ \\
\hline $\begin{array}{l}\text { Marriott et al. } \\
(2008)^{(9)} \text { UK (3.5) }\end{array}$ & $\begin{array}{l}n 50 \\
12 \text { months } \\
\text { Infants }\end{array}$ & $\begin{array}{l}\text { FFQ } v .4 \text { d WDR } \\
\text { seventy-eight items. } \\
\text { HH measures }\end{array}$ & $\begin{array}{l}\text { Energy adjusted } \\
\text { CC } 0.58\end{array}$ & $\begin{array}{l}\text { Median }(\mu \mathrm{g}) \\
\text { FFQ } 559 \\
\text { WDR } 400\end{array}$ \\
\hline $\begin{array}{l}\text { Parrish et al. } \\
(2003)^{(22)} \text { USA (3.5) }\end{array}$ & $\begin{array}{l}n 38 \\
1-3 \text { years } \\
\text { Infants } \\
\text { Preschool children }\end{array}$ & $\begin{array}{l}\text { FFQ } v \text {. BM } 111 \text { items. } \\
\text { Standard reference } \\
\text { portions }\end{array}$ & CC 0.01 & \\
\hline & & $24 \mathrm{HR} v . \mathrm{BM}$ & CC 0.35 & \\
\hline \multirow[t]{2}{*}{$\begin{array}{l}\text { Holmes et al. } \\
(2008)^{(10)} \text { UK (3) }\end{array}$} & $\begin{array}{l}n 124 \\
2-17 \text { years } \\
\text { Preschool children } \\
\text { Children }\end{array}$ & $24 \mathrm{HR}$ v. $4 \mathrm{~d}$ WDR & $\begin{array}{l}\text { Males-Mean difference } \\
2-10 \text { years } 77(P=0.058) \\
11-17 \text { years } 65(P=0.280)\end{array}$ & $\begin{array}{l}\text { Females-Mean difference } \\
2-10 \text { years } 155(P=0.008) \\
11-17 \text { years } 57(P=0.633)\end{array}$ \\
\hline & Adolescents & FC v. $4 \mathrm{~d}$ WDR & $\begin{array}{l}\text { Males-Mean difference } \\
2-10 \text { years } 68(P=0.135)\end{array}$ & $\begin{array}{l}\text { Females-Mean difference } \\
2-10 \text { years } 75(P=0.076)\end{array}$ \\
\hline
\end{tabular}




\begin{tabular}{|c|c|c|c|c|c|c|}
\hline \multirow[t]{2}{*}{$\begin{array}{l}\text { Author/year } \\
\text { publication/country/ } \\
\text { (quality index) }\end{array}$} & \multirow[t]{2}{*}{ n/age group } & \multirow{2}{*}{ 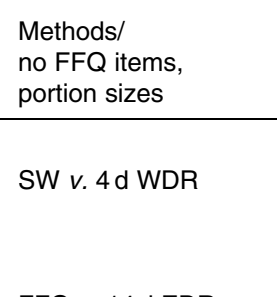 } & \multicolumn{4}{|c|}{ Correlation between methods/other statistics } \\
\hline & & & $\begin{array}{l}11-17 \text { years } 19(P=0.808) \\
\text { Males-Mean difference } \\
2-10 \text { years }-4(P=0.935) \\
11-17 \text { years }-21 \\
(P=0.821)\end{array}$ & $\begin{array}{l}11-17 \text { years }-25(P=0.851) \\
\text { Females-Mean difference } \\
2-10 \text { years } 44(P=0.299) \\
11-17 \text { years }-27(P=0.826)\end{array}$ & & \\
\hline $\begin{array}{l}\text { Arnold et al. } \\
(1995)^{(31)} \text { Canada } \\
(3.5)\end{array}$ & $\begin{array}{l}n 707 \\
7-12 \text { years } \\
\text { Children }\end{array}$ & $\begin{array}{l}\text { FFQ } v .14 \mathrm{~d} \text { EDR } \\
160 \text { items. Standard } \\
\text { reference portions }\end{array}$ & Pearson CC 0.30 & & & \\
\hline $\begin{array}{l}\text { Lillegaard et al. } \\
(2007)^{(12)} \text { Norway } \\
(3.5)\end{array}$ & $\begin{array}{l}n 100 \\
9 \text { years old } \\
\text { Children }\end{array}$ & $\begin{array}{l}\text { PFD v. } 4 \text { d WDR } \\
277 \text { items. HH } \\
\text { measures or colour } \\
\text { photographs }\end{array}$ & $\begin{array}{l}\text { Boys-Median }\left(P_{25-} P_{75}\right)(\mu g) \\
\text { PFD } 854(489,1304) \\
\text { WDR } 800(498,1173) \\
\text { CC } 0.62^{\star \star \star}\end{array}$ & $\begin{array}{l}\text { Girls-Median }\left(P_{25-} P_{75}\right)(\mu \mathrm{g}) \\
\text { PFD } 876(636,1323) \\
\text { WDR } 695(506,987) \\
\text { CC } 0.40^{* *}\end{array}$ & & \\
\hline $\begin{array}{l}\text { Rockett et al. } \\
(1997)^{(29)} \text { USA (3.5) }\end{array}$ & $\begin{array}{l}n 261 \\
9-18 \text { years } \\
\text { Children } \\
\text { Adolescents }\end{array}$ & $\begin{array}{l}\text { YAQ } v \text {. three } 24 \mathrm{HR} \\
131 \text { items. Standard } \\
\text { reference portions }\end{array}$ & $\begin{array}{l}9-13 \text { years CC } 0.44 \\
14-18 \text { years CC } 0.51\end{array}$ & $\begin{array}{l}\text { Mean (SD) (IU) } \\
24 \text { HR } 2917(2442) \\
\text { YAQ } 3436(2109)\end{array}$ & & \\
\hline $\begin{array}{l}\text { Slater et al. } \\
(2003)^{(20)} \text { Brazil (4) }\end{array}$ & $\begin{array}{l}n 79 \\
14-18 \text { years } \\
\text { Adolescents }\end{array}$ & $\begin{array}{l}\text { FFQ } v \text {. three } 24 \mathrm{HR} \\
76 \text { items. Single } \\
\text { reference portion } \\
\text { size listed }\end{array}$ & $\begin{array}{l}\text { Non-adjusted } 0.28^{\star} \\
\text { Energy adjusted } 0.06 \\
\text { Deattenuated } 0.10\end{array}$ & $\begin{array}{l}\text { Mean }(\mathrm{sD})(\mu \mathrm{g}) \\
24 \text { HR } 745(831) \\
\text { FFQ } 614(243)\end{array}$ & & \\
\hline $\begin{array}{l}\text { Andersen et al. } \\
(1995)^{(33)} \text { Norway } \\
(3) \\
\beta \text {-Carotene }\end{array}$ & $\begin{array}{l}n 49 \\
\text { Adolescents }\end{array}$ & $\begin{array}{l}\text { FFQ } v .7 \mathrm{~d} \mathrm{WDR} \\
190 \text { items. } \mathrm{HH} \\
\text { measures }\end{array}$ & $\begin{array}{l}\text { Unadjusted } 0.30 \\
\text { Energy adjusted } 0.39\end{array}$ & $\begin{array}{l}\text { Median FFQ: } 1381(\mu \mathrm{g}) \\
\text { Median WDR: } 787(\mu \mathrm{g})\end{array}$ & & \\
\hline $\begin{array}{l}\text { Parrish et al. } \\
(2003)^{(22)} \text { USA (3.5) }\end{array}$ & $\begin{array}{l}n 38 \\
1-3 \text { years } \\
\text { Infants } \\
\text { Preschool children }\end{array}$ & $\begin{array}{l}\text { FFQ } v . \mathrm{BM} \\
111 \text { items. Standard } \\
\text { reference portions }\end{array}$ & CC 0.13 & & & \\
\hline $\begin{array}{l}\text { Arnold et al. } \\
(1995)^{(31)} \text { Canada } \\
(3.5)\end{array}$ & $\begin{array}{l}n 707 \\
7-12 \text { years } \\
\text { Children }\end{array}$ & $\begin{array}{l}\text { FFQ } v .14 \mathrm{~d} \text { EDR } \\
160 \text { items. Standard } \\
\text { reference portions }\end{array}$ & Pearson CC 0.53 & & & \\
\hline Vitamin D & & & & & & \\
\hline $\begin{array}{l}\text { Marriott et al. } \\
(2008)^{(8)} \text { UK (3.5) }\end{array}$ & $\begin{array}{l}n 50 \\
6 \text { months } \\
\text { Infants }\end{array}$ & $\begin{array}{l}\text { FFQ } v .4 \mathrm{~d} \text { WDR } \\
\text { thirty-four items. HH } \\
\text { measures }\end{array}$ & $\begin{array}{l}\text { Energy adjusted } \\
\text { CC } 0.83\end{array}$ & $\begin{array}{l}\text { Median }(\mu \mathrm{g}) \\
\text { FFQ } 9 \cdot 3 \\
\text { WDR } 8.3\end{array}$ & & \\
\hline $\begin{array}{l}\text { Marriott et al. } \\
(2008)^{(9)} \text { UK (3.5) }\end{array}$ & $\begin{array}{l}n 50 \\
12 \text { months } \\
\text { Infants }\end{array}$ & $\begin{array}{l}\text { FFQ } v .4 \mathrm{~d} \text { WDR } \\
\text { seventy-eight items. } \\
\text { HH measures }\end{array}$ & $\begin{array}{l}\text { Energy adjusted } \\
\text { CC } 0.40\end{array}$ & $\begin{array}{l}\text { Median }(\mu \mathrm{g}) \\
\text { FFQ } 6.29 \\
\text { WDR } 1.54\end{array}$ & & \\
\hline $\begin{array}{l}\text { Andersen et al. } \\
(2003)^{(2)} \text { Norway } \\
(4.5)\end{array}$ & $\begin{array}{l}n 64 \\
12 \text { months } \\
\text { Infants }\end{array}$ & $\begin{array}{l}\text { FFQ } v .7 \mathrm{~d} \text { WDR } \\
140 \text { items. HH } \\
\text { measures or food } \\
\text { photographs }\end{array}$ & $\begin{array}{l}\text { Absolute intake } \\
\text { CC } 0.18\end{array}$ & $\begin{array}{l}\text { Median }\left(P_{25}-P_{75}\right)(\mu \mathrm{g}) \\
\text { FFQ 2.3 }(1 \cdot 1-4 \cdot 2) \\
\text { WDR } 1 \cdot 1(0 \cdot 4-1 \cdot 9)^{\star \star \star}\end{array}$ & & \\
\hline $\begin{array}{l}\text { Marshall et al. } \\
(2003)^{(21)} \text { USA (4) }\end{array}$ & $\begin{array}{l}n 240 \\
6-12 \text { months } \\
3-5 \text { years } \\
\text { Infants } \\
\text { Preschool children }\end{array}$ & $\begin{array}{l}\text { FFQ v. 3d WDR } \\
\text { seven items. } \\
\text { Estimated usual } \\
\text { serving size }\end{array}$ & $\begin{array}{l}6 \text { months } \\
\text { CC } 0 \cdot 80^{\star \star \star} \\
\text { Mean }(\mathrm{sD}) \mu \mathrm{g} \\
\text { FFQ } 6 \cdot 4(3 \cdot 4) \\
\text { WDR } 5 \cdot 6(2 \cdot 8)\end{array}$ & $\begin{array}{l}12 \text { months } \\
\text { CC } 0 \cdot 60^{\star \star \star} \\
\text { Mean }(\mathrm{sD}) \mu \mathrm{g} \\
\text { FFQ } 6 \cdot 1(3 \cdot 3) \\
\text { WDR } 5 \cdot 6(2 \cdot 8)\end{array}$ & $\begin{array}{l}3 \text { years } \\
\text { CC } 0.74^{\star \star \star} \\
\text { Mean }(\mathrm{SD}) \mu \mathrm{g} \\
\text { FFQ } 3 \cdot 2(2 \cdot 3) \\
\text { WDR } 3 \cdot 7(2 \cdot 3)\end{array}$ & $\begin{array}{l}5 \text { years } \\
\text { CC } 0.63^{\star \star \star} \\
\text { Mean }(\mathrm{SD}) \mu \mathrm{g} \\
\text { FFQ } 3.3(2 \cdot 1) \\
\text { WDR } 3.4(1.9)\end{array}$ \\
\hline $\begin{array}{l}\text { Parrish et al. } \\
(2003)^{(22)} \text { USA (3.5) }\end{array}$ & $\begin{array}{l}n 68 \\
1-3 \text { years }\end{array}$ & $\begin{array}{l}\text { FFQ } v . \text { BM } \\
111 \text { items. Standard }\end{array}$ & $\mathrm{CC} 0.00$ & & & \\
\hline
\end{tabular}




\begin{tabular}{|c|c|c|c|c|}
\hline \multirow[t]{2}{*}{$\begin{array}{l}\text { Author/year } \\
\text { publication/country/ } \\
\text { (quality index) }\end{array}$} & n/age group & $\begin{array}{l}\text { Methods/ } \\
\text { no FFQ items, } \\
\text { portion sizes }\end{array}$ & \multicolumn{2}{|c|}{ Correlation between methods/other statistics } \\
\hline & $\begin{array}{l}\text { Infants } \\
\text { Preschool children }\end{array}$ & $\begin{array}{l}\text { reference portions } \\
24 \mathrm{HR} v \text {. BM }\end{array}$ & $\mathrm{CC} 0.17$ & \\
\hline $\begin{array}{l}\text { Andersen et al. } \\
(2004)^{(18,19)} \\
\text { Norway (5) }\end{array}$ & $\begin{array}{l}n 187 \\
2 \text { years old } \\
\text { Preschool children }\end{array}$ & $\begin{array}{l}\text { FFQ } v .7 \mathrm{~d} \text { WDR } \\
125 \text { items. HH } \\
\text { measures or food } \\
\text { photographs }\end{array}$ & $\begin{array}{l}\text { Absolute intake } \\
\text { CC } 0.48^{\star \star \star}\end{array}$ & $\begin{array}{l}\text { Median }\left(P_{25}-P_{75}\right)(\mu \mathrm{g}) \\
\text { FFQ } 9.1(5 \cdot 8-13.7) \\
\text { WDR } 3.9(1.6-7 \cdot 3)^{\star \star \star}\end{array}$ \\
\hline $\begin{array}{l}\text { Lillegaard et al. } \\
(2007)^{(12)} \text { Norway } \\
(3 \cdot 5) \\
\text { Andersen et al. }\end{array}$ & $\begin{array}{l}n 100 \\
9 \text { years old } \\
\text { Children } \\
n 49\end{array}$ & $\begin{array}{l}\text { PFD } v .4 \mathrm{~d} \text { WDR } \\
277 \text { items. HH } \\
\text { measures or colour } \\
\text { photographs } \\
\text { FFQ } v .7 \mathrm{~d} \text { WDR }\end{array}$ & $\begin{array}{l}\text { Boys-Median }\left(\mathrm{P}_{25-} \mathrm{P}_{75}\right)(\mu \mathrm{g}) \\
\text { PFD } 3.0(1.5,6 \cdot 2) \\
\text { WDR } 2 \cdot 1(1 \cdot 2,3 \cdot 7) \\
\text { CC } 0.51^{\star \star \star} \\
\text { Unadjusted } 0.14\end{array}$ & $\begin{array}{l}\text { Girls-Median }\left(\mathrm{P}_{25-} \mathrm{P}_{75}\right)(\mu \mathrm{g}) \\
\text { PFD } 3.4(2 \cdot 0,5.9) \\
\text { WDR } 2 \cdot 2(1.5,4 \cdot 2) \\
\text { CC } 0.52^{\star \star \star} \\
\text { Median FFQ: } 4.9(\mu \mathrm{g})\end{array}$ \\
\hline $\begin{array}{l}(1995)^{(33)} \text { Norway } \\
(3)\end{array}$ & Adolescents & $\begin{array}{l}190 \text { items. HH } \\
\text { measures }\end{array}$ & Energy adjusted 0.21 & Median WDR: $2 \cdot 2(\mu \mathrm{g})$ \\
\hline \multicolumn{5}{|l|}{ Vitamin E } \\
\hline $\begin{array}{l}\text { Marriott et al. } \\
(2008)^{(8)} \text { UK (3.5) }\end{array}$ & $\begin{array}{l}n 50 \\
6 \text { months } \\
\text { Infants }\end{array}$ & $\begin{array}{l}\text { FFQ } v .4 \mathrm{~d} \text { WDR } \\
\text { thirty-four items. HH } \\
\text { measures }\end{array}$ & $\begin{array}{l}\text { Energy adjusted } \\
\text { CC } 0.83\end{array}$ & $\begin{array}{l}\text { Median }(\mathrm{mg}) \\
\text { FFQ } 6.0 \\
\text { WDR } 5.3\end{array}$ \\
\hline $\begin{array}{l}\text { Marriott et al. } \\
(2008)^{(9)} \text { UK (3.5) }\end{array}$ & $\begin{array}{l}n 50 \\
12 \text { months } \\
\text { Infants }\end{array}$ & $\begin{array}{l}\text { FFQ } v .4 \text { d WDR } \\
\text { seventy-eight items. } \\
\text { HH measures }\end{array}$ & $\begin{array}{l}\text { Energy adjusted } \\
\text { CC } 0.27\end{array}$ & $\begin{array}{l}\text { Median }(\mu \mathrm{g}) \\
\text { FFQ } 6 \cdot 13 \\
\text { WDR } 4.11\end{array}$ \\
\hline $\begin{array}{l}\text { Andersen et al. } \\
(2003)^{(2)} \text { Norway } \\
(4.5)\end{array}$ & $\begin{array}{l}n 64 \\
12 \text { months } \\
\text { Infants }\end{array}$ & $\begin{array}{l}\text { FFQ } v .7 \mathrm{~d} \text { WDR } \\
140 \text { items. HH } \\
\text { measures or food } \\
\text { photographs }\end{array}$ & $\begin{array}{l}\text { Absolute intake } \\
\text { CC } 0.32\end{array}$ & $\begin{array}{l}\text { Median }\left(\mathrm{P}_{25}-\mathrm{P}_{75}\right)(\mathrm{mg}) \\
\text { FFQ 4.0 }(3.1-5 \cdot 6) \\
\text { WDR 2.9 }(2.3-4 \cdot 2)^{\star \star \star}\end{array}$ \\
\hline $\begin{array}{l}\text { Parrish et al. } \\
(2003)^{(22)} \text { USA (3.5) }\end{array}$ & $\begin{array}{l}n 38 \\
1-3 \text { years } \\
\text { Infants } \\
\text { Preschool children }\end{array}$ & $\begin{array}{l}\text { FFQ } v . \mathrm{BM} \\
111 \text { items. Standard } \\
\text { reference portions } \\
24 \mathrm{HR} v . \mathrm{BM}\end{array}$ & CC 0.30 & \\
\hline $\begin{array}{l}\text { Blum et al. } \\
(1999)^{(24)} \text { USA (2.5) }\end{array}$ & $\begin{array}{l}n 233 \\
1-5 \text { years Infants } \\
\text { Preschool children }\end{array}$ & $\begin{array}{c}\text { FFQ } v \text {. three } 24 \mathrm{HR} \\
\text { eighty-four items. }\end{array}$ & CC 0.56 & $\begin{array}{l}\text { Mean }(\mathrm{sD})(\mathrm{mg}) \\
24 \mathrm{HR} 8(5) \\
\text { FFQ } 10(7)\end{array}$ \\
\hline $\begin{array}{l}\text { Andersen et al. } \\
(2004)^{(18,19)}\end{array}$ & $\begin{array}{l}n 187 \\
2 \text { years old }\end{array}$ & $\begin{array}{l}\text { FFQ v. } 7 \mathrm{~d} \text { WDR } \\
125 \text { items. HH }\end{array}$ & $\begin{array}{l}\text { Absolute intake } \\
\text { CC } 0.48^{\star \star \star}\end{array}$ & $\begin{array}{l}\text { Median }\left(\mathrm{P}_{25}-\mathrm{P}_{75}\right)(\mathrm{mg}) \\
\text { FFQ 9.3 }(6 \cdot 2-12 \cdot 7)\end{array}$ \\
\hline Norway (5) & Preschool children & $\begin{array}{l}\text { measures or food } \\
\text { photographs }\end{array}$ & & WDR $4.7(2 \cdot 9-7 \cdot 2)^{\star \star \star}$ \\
\hline $\begin{array}{l}\text { Byers et al. } \\
(1993)^{(35)} \text { USA (2.5) }\end{array}$ & $\begin{array}{l}n 97 \\
6-10 \text { years } \\
\text { Children }\end{array}$ & $\begin{array}{l}\text { FFQ } v . \mathrm{BM} \\
111 \text { items }\end{array}$ & $\begin{array}{l}\text { Crude } 0.32 \\
\text { Adjusted } 0.32\end{array}$ & \\
\hline $\begin{array}{l}\text { Bellù et al. } \\
(1995)^{(32)} \text { Italy (3.5) }\end{array}$ & $\begin{array}{l}n 88 \\
9-12 \text { years } \\
\text { Children }\end{array}$ & $\begin{array}{l}\text { FFQ } v .7 \mathrm{~d} \text { EDR } \\
116 \text { items. Reference } \\
\text { portion sizes }\end{array}$ & $\begin{array}{l}\text { Raw nutrient } 0.31 \\
\text { Nutrient density } 0.38\end{array}$ & $\begin{array}{l}\text { Mean (SD) } \\
\text { EDR } 0.67(0.21) \\
\text { FFQ } 0.57(0.22)\end{array}$ \\
\hline $\begin{array}{l}\text { Rockett et al. } \\
(1997)^{(29)} \text { USA (3.5) }\end{array}$ & $\begin{array}{l}n 26 \\
19-18 \text { years } \\
\text { Children- } \\
\text { Adolescents }\end{array}$ & $\begin{array}{l}\text { YAQ } v .3-24 \mathrm{HR} \\
131 \text { items. Standard } \\
\text { reference portions }\end{array}$ & $\begin{array}{l}9-13 \text { years CC } 0.50 \\
14-18 \text { years } C C 0.55\end{array}$ & $\begin{array}{l}\text { Mean }(\mathrm{SD})(\mathrm{mg}) \\
24 \mathrm{HR} 13(22) \\
\text { YAQ } 13(11)\end{array}$ \\
\hline
\end{tabular}




\begin{tabular}{|c|c|c|c|c|}
\hline \multirow{2}{*}{$\begin{array}{l}\text { Author/year } \\
\text { publication/country/ } \\
\text { (quality index) } \\
\text { Thiamin }\end{array}$} & \multirow[t]{2}{*}{$n / a g e$ group } & \multirow[t]{2}{*}{$\begin{array}{l}\text { Methods/ } \\
\text { no FFQ items, } \\
\text { portion sizes }\end{array}$} & \multicolumn{2}{|c|}{ Correlation between methods/other statistics } \\
\hline & & & & \multirow[b]{2}{*}{$\begin{array}{l}\text { Median }(\mathrm{mg}) \\
\text { FFQ } 0.65 \\
\text { WDR } 0.60\end{array}$} \\
\hline $\begin{array}{l}\text { Marriott et al. } \\
(2008)^{(8)} \text { UK (3.5) }\end{array}$ & $\begin{array}{l}n 50 \\
6 \text { months } \\
\text { Infants }\end{array}$ & $\begin{array}{l}\text { FFQ } v .4 \mathrm{~d} \text { WDR } \\
\text { thirty-four items. HH } \\
\text { measures }\end{array}$ & $\begin{array}{l}\text { Energy adjusted } \\
\text { CC } 0.89\end{array}$ & \\
\hline $\begin{array}{l}\text { Marriott et al. } \\
(2008)^{(9)} \text { UK (3.5) }\end{array}$ & $\begin{array}{l}n 50 \\
12 \text { months } \\
\text { Infants }\end{array}$ & $\begin{array}{l}\text { FFQ v. } 4 \text { d WDR } \\
\text { seventy-eight items. } \\
\text { HH measures }\end{array}$ & $\begin{array}{l}\text { Energy adjusted } \\
\text { CC } 0.53\end{array}$ & $\begin{array}{l}\text { Median }(\mu \mathrm{g}) \\
\text { FFQ } 0.96 \\
\text { WDR } 0.75\end{array}$ \\
\hline $\begin{array}{l}\text { Andersen et al. } \\
(2003)^{(2)} \text { Norway } \\
(4.5)\end{array}$ & $\begin{array}{l}n 64 \\
12 \text { months } \\
\text { Infants }\end{array}$ & $\begin{array}{l}\text { FFQ } v .7 \mathrm{~d} \text { WDR } \\
140 \text { items. HH } \\
\text { measures or food } \\
\text { photographs }\end{array}$ & $\begin{array}{l}\text { Absolute intake } \\
\text { CC } 0.41\end{array}$ & $\begin{array}{l}\text { Median }\left(\mathrm{P}_{25}-\mathrm{P}_{75}\right)(\mathrm{mg}) \\
\text { FFQ } 0.7(0.59-0.88) \\
\text { WDR } 0.56(0.44-0.61)^{\star \star *}\end{array}$ \\
\hline $\begin{array}{l}\text { Blum et al. } \\
(1999)^{(24)} \text { USA (2.5) }\end{array}$ & $\begin{array}{l}n 233 \\
1-5 \text { years } \\
\text { Infants } \\
\text { Preschool children }\end{array}$ & $\begin{array}{l}\text { FFQ } v \text {. three } 24 \mathrm{HR} \\
\text { eighty-four items. }\end{array}$ & $\mathrm{CC} 0.57$ & $\begin{array}{l}\text { Mean }(\mathrm{sD})(\mathrm{mg}) \\
24 \mathrm{HR} 2(1) \\
\text { FFQ } 2(1)\end{array}$ \\
\hline $\begin{array}{l}\text { Andersen et al. } \\
(2004)^{(18,19)} \\
\text { Norway (5) }\end{array}$ & $\begin{array}{l}n 187 \\
2 \text { years old } \\
\text { Preschool children }\end{array}$ & $\begin{array}{l}\text { FFQ } v .7 \mathrm{~d} \text { WDR } \\
125 \text { items. HH } \\
\text { measures or food } \\
\text { photographs }\end{array}$ & $\begin{array}{l}\text { Absolute intake } \\
\text { CC } 0.52^{\star \star \star}\end{array}$ & $\begin{array}{l}\text { Median }\left(\mathrm{P}_{25}-\mathrm{P}_{75}\right)(\mathrm{mg}) \\
\text { FFQ 1.12 }(0.86-1.64) \\
\text { WDR } 0.66(0.55-0.91)^{\star \star \star}\end{array}$ \\
\hline \multirow[t]{3}{*}{$\begin{array}{l}\text { Holmes et al. } \\
(2008)^{(10)} \text { UK (3) }\end{array}$} & $\begin{array}{l}n 124 \\
2-17 \text { years } \\
\text { Preschool children } \\
\text { Children } \\
\text { Adolescents }\end{array}$ & $24 \mathrm{HR}$ v. 4 d WDR & $\begin{array}{l}\text { Males-Mean difference } \\
2-10 \text { years }-0.1(P=0.480) \\
11-17 \text { years } 0.4(P=0.142)\end{array}$ & $\begin{array}{l}\text { Females-Mean difference } \\
2-10 \text { years } 0.2(P=0.024) \\
11-17 \text { years } 0.1(P=0.041)\end{array}$ \\
\hline & & FC v. 4d WDR & $\begin{array}{l}\text { Males-Mean difference } \\
2-10 \text { years }-0.2(P=0.574) \\
11-17 \text { years } 0.2(P=0.433)\end{array}$ & $\begin{array}{l}\text { Females-Mean difference } \\
2-10 \text { years } 0.3(P=0.000)\end{array}$ \\
\hline & & SW v. $4 \mathrm{~d}$ WDR & $\begin{array}{l}11-17 \text { years } 0.2(P=0.433) \\
\text { Males-Mean difference } \\
2-10 \text { years }-0.8(P=0.243) \\
11-17 \text { years }-0.1(P=0.393)\end{array}$ & $\begin{array}{l}11-17 \text { years } 0.2(P=0.063) \\
\text { Females-Mean difference } \\
2-10 \text { years } 0.0(P=0.575) \\
11-17 \text { years } 0.1(P=0.658)\end{array}$ \\
\hline $\begin{array}{l}\text { Räsänen L. } \\
(1979)^{(39)} \text { Finland } \\
(5)\end{array}$ & $\begin{array}{l}n 741 \\
5-13 \text { years } \\
\text { Preschool children } \\
\text { Children } \\
\text { Adolescents }\end{array}$ & $24 \mathrm{HR} v . \mathrm{DH}$ & CC 0.46 & Mean DH: $1.9 ; 24$ HR: 1.3 \\
\hline $\begin{array}{l}\text { Arnold et al. } \\
(1995)^{(31)} \text { Canada } \\
(3.5)\end{array}$ & $\begin{array}{l}n 707 \\
7-12 \text { years } \\
\text { Children }\end{array}$ & $\begin{array}{l}\text { FFQ } v .14 \mathrm{~d} \text { EDR } \\
160 \text { items. Standard } \\
\text { reference portions }\end{array}$ & Pearson CC $0.35 \dagger$ & \\
\hline $\begin{array}{l}\text { Lillegaard et al. } \\
(2007)^{(12)} \text { Norway } \\
(3.5)\end{array}$ & $\begin{array}{l}n 100 \\
9 \text { years old } \\
\text { Children }\end{array}$ & $\begin{array}{l}\text { PFD v. } 4 \text { d WDR } \\
277 \text { items. HH } \\
\text { measures or colour } \\
\text { photographs }\end{array}$ & $\begin{array}{l}\text { Boys- Median }\left(P_{25-} P_{75}\right)(\mathrm{mg}) \\
\text { PFD } 1.1(0.9,1.4) \\
\text { WDR } 1.1(0.9,1.4) \\
\text { CC } 0.53^{\star \star \star}\end{array}$ & $\begin{array}{l}\text { Girls-Median }\left(\mathrm{P}_{25-} \mathrm{P}_{75}\right)(\mathrm{mg}) \\
\text { PFD } 1 \cdot 1(0 \cdot 9,1.5) \\
\text { WDR } 1.0(0 \cdot 8,1 \cdot 2) \\
\text { CC } 0.40^{\star \star}\end{array}$ \\
\hline $\begin{array}{l}\text { Bellù et al. } \\
(1995)^{(32)} \text { Italy (3.5) }\end{array}$ & $\begin{array}{l}n 88 \\
9-12 \text { years } \\
\text { Children }\end{array}$ & $\begin{array}{l}\text { FFQ } v .7 \mathrm{~d} \text { EDR } \\
116 \text { items. Reference } \\
\text { portion sizes }\end{array}$ & $\begin{array}{l}\text { Raw nutrient } 0.24 \\
\text { Nutrient density } 0.27\end{array}$ & $\begin{array}{l}\text { Mean (SD) } \\
\text { EDR } 0.61(0.78) \\
\text { FFQ } 1.41(0.78)\end{array}$ \\
\hline $\begin{array}{l}\text { Rockett et al. } \\
(1997)^{(29)} \text { USA (3.5) }\end{array}$ & $\begin{array}{l}n 261 \\
9-18 \text { years } \\
\text { Children } \\
\text { Adolescents }\end{array}$ & $\begin{array}{l}\text { YAQ } v \text {. three } 24 \mathrm{HR} \\
131 \text { items. Standard } \\
\text { reference portions }\end{array}$ & $\begin{array}{l}9-13 \text { years CC } 0.49 \\
14-18 \text { years CC } 0.54\end{array}$ & $\begin{array}{l}\text { Mean }(\mathrm{SD})(\mathrm{mg}) \\
24 \mathrm{HR} 2 \cdot 3(1.8) \\
\text { YAQ } 2 \cdot 0(0 \cdot 8)\end{array}$ \\
\hline
\end{tabular}




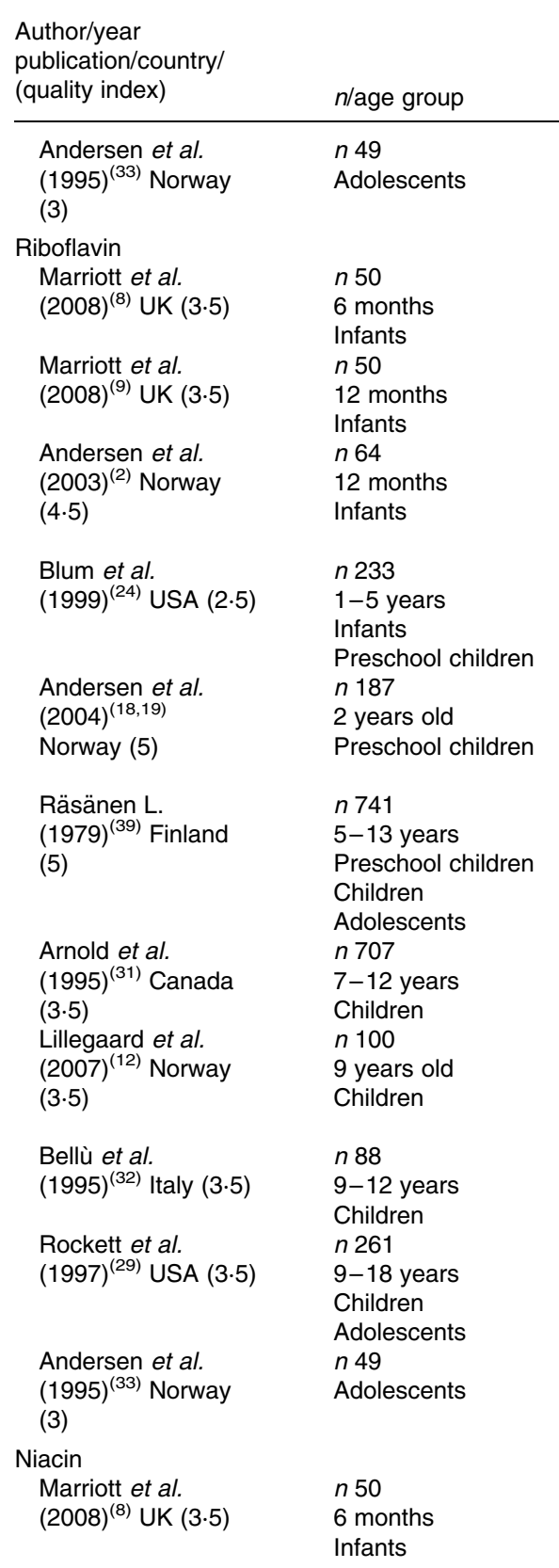

Methods/

no FFQ items, portion sizes

Correlation between methods/other statistics

$\begin{array}{lll}\text { FFQ } v .7 \mathrm{~d} \mathrm{WDR} & \text { Unadjusted } 0.53 & \text { Median FFQ: } 1.58(\mathrm{mg}) \\ 190 \text { items. HH } & \text { Energy adjusted } 0.53 & \text { Median WDR: } 1.14(\mathrm{mg})\end{array}$

measures

Energy adjusted

FFQ v. 4 d WDR

thirty-four items. $\mathrm{HH}$

CC 0.87

FFQ $v$. 4 d WDR

seventy-eight items.

$\mathrm{HH}$ measures

FFQ v. $7 \mathrm{~d}$ WDR

140 items. $\mathrm{HH}$

measures or food

photographs

FFQ $v$. three $24 \mathrm{HR}$

eighty-four items.

FFQ v. $7 \mathrm{~d}$ WDR

125 items. $\mathrm{HH}$

measures or food

photographs

Energy adjusted

CC 0.51

Absolute intake

CC 0.58

CC 0.56

$24 \mathrm{HR} v$. DH

Median (mg)

FFQ 1.2

WDR 1.2

Median $(\mu \mathrm{g})$

FFQ 1.70

WDR 1.43

Median $\left(\mathrm{P}_{25}-\mathrm{P}_{75}\right)(\mathrm{mg})$

FFQ $1.02(0.72-1.34)$

WDR $0.78(0.58-1.02)^{\star \star \star}$

Mean (sD) (mg)

24 HR 2 (1)

FFQ 3 (1)

Absolute intake

CC $0.51^{\star \star \star}$

Median $\left(\mathrm{P}_{25}-\mathrm{P}_{75}\right)(\mathrm{mg})$

FFQ $1.63(1.24-2.24)$

WDR $1.06(0.84-1.33)^{\star * *}$

Mean DH: 3.3; 24 HR: $2 \cdot 3$

FFQ $v .14$ d EDR

160 items. Standard

reference portions

PFD v. 4 d WDR

277 items. $\mathrm{HH}$

measures or colour

photographs

FFQ $v .7 d$ EDR

116 items. Reference

portion sizes

YAQ $v$. three $24 \mathrm{HR}$

131 items. Standard

Pearson CC $0.36 \dagger$

reference portions

Boys- Median $\left(\mathrm{P}_{25-} \mathrm{P}_{75}\right)(\mathrm{mg})$

Girls-Median $\left(\mathrm{P}_{25-} \mathrm{P}_{75}\right)(\mathrm{mg})$

PFD 1.5 (1.1. 2.0)

WDR $1.3(1 \cdot 1,1 \cdot 8)$

CC $0.56^{* * \star}$

Raw nutrient 0.26

Nutrient density 0.22

PFD $1.4(1.1,1.9)$

WDR $1.2(0.9,1 \cdot 6)$

CC $0.56^{* * *}$

Mean (SD)

EDR $0.64(0.16)$

FFQ $0.64(0.11)$

Mean (SD) (mg)

$9-13$ years CC 0.50
$14-18$ years CC 0.62

24 HR $2.7(1.5)$

FFQ v. $7 \mathrm{~d}$ WDR

YAQ $2.7(1 \cdot 0)$

Unadjusted 0.57

Median FFQ: $2.01(\mathrm{mg})$

190 items. HH

Energy adjusted 0.65

Median WDR: $1.47(\mathrm{mg})$

FFQ v. 4 d WDR

thirty-four items.

Energy adjusted

CC 0.88

Median (mg)

FFQ 8.1

WDR 8.0 
Table 3. Continued

\begin{tabular}{|c|c|c|c|c|}
\hline \multirow{2}{*}{$\begin{array}{l}\begin{array}{l}\text { Author/year } \\
\text { publication/country/ } \\
\text { (quality index) }\end{array} \\
\begin{array}{l}\text { Marriott et al. } \\
(2008)^{(9)} \text { UK (3.5) }\end{array}\end{array}$} & \multirow{2}{*}{$\begin{array}{l}n / \text { age group } \\
n 50 \\
12 \text { months } \\
\text { Infants }\end{array}$} & \multirow{2}{*}{$\begin{array}{l}\text { Methods/ } \\
\text { no FFQ items, } \\
\text { portion sizes } \\
\text { FFQ v. } 4 \text { d WDR } \\
\text { seventy-eight items. } \\
\text { HH measures }\end{array}$} & \multicolumn{2}{|c|}{ Correlation between methods/other statistics } \\
\hline & & & $\begin{array}{l}\text { Energy adjusted } \\
\text { CC } 0.60\end{array}$ & $\begin{array}{l}\text { Median }(\mu \mathrm{g}) \\
\text { FFQ } 10.52 \\
\text { WDR } 7.48\end{array}$ \\
\hline $\begin{array}{l}\text { Blum et al. } \\
(1999)^{(24)} \text { USA (2.5) }\end{array}$ & $\begin{array}{l}n 233 \\
1-5 \text { years } \\
\text { Infants } \\
\text { Preschool children }\end{array}$ & $\begin{array}{l}\text { FFQ } v \text {. three } 24 \mathrm{HR} \\
84 \text { items. }\end{array}$ & CC 0.55 & $\begin{array}{l}\text { Mean }(\mathrm{sD})(\mathrm{mg}) \\
24 \mathrm{HR} 19(8) \\
\text { FFQ } 21(8)\end{array}$ \\
\hline $\begin{array}{l}\text { Räsänen L. } \\
(1979)^{(39)} \text { Finland } \\
(5)\end{array}$ & $\begin{array}{l}n 741 \\
5-13 \text { years } \\
\text { Preschool children } \\
\text { Children } \\
\text { Adolescents }\end{array}$ & $24 \mathrm{HR} v . \mathrm{DH}$ & $\mathrm{CC} 0.36$ & Mean DH: 16.2; 24 HR: $11 \cdot 2$ \\
\hline $\begin{array}{l}\text { Rockett et al. } \\
(1997)^{(29)} \text { USA (3.5) }\end{array}$ & $\begin{array}{l}n 261 \\
9-18 \text { years } \\
\text { Children } \\
\text { Adolescents }\end{array}$ & $\begin{array}{r}\text { YAQ } v \text {. three } 24 \mathrm{HR} \\
131 \text { items. Standard } \\
\text { reference portions }\end{array}$ & $\begin{array}{l}9-13 \text { years CC } 0.45 \\
14-18 \text { years CC } 0.50\end{array}$ & $\begin{array}{l}\text { Mean }(\mathrm{SD})(\mathrm{mg}) \\
24 \mathrm{HR} 26(15) \\
\text { YAQ } 25(10)\end{array}$ \\
\hline \multicolumn{5}{|l|}{ Vitamin $\mathrm{B}_{6}$} \\
\hline $\begin{array}{l}\text { Marriott et al. } \\
(2008)^{(8)} \text { UK (3.5) }\end{array}$ & $\begin{array}{l}n 50 \\
6 \text { months } \\
\text { Infants }\end{array}$ & $\begin{array}{l}\text { FFQ } v .4 \mathrm{~d} \text { WDR } \\
\text { thirty-four items. HH } \\
\text { measures }\end{array}$ & $\begin{array}{l}\text { Energy adjusted } \\
\text { CC } 0.84\end{array}$ & $\begin{array}{l}\text { Median }(\mathrm{mg}) \\
\text { FFQ } 0.60 \\
\text { WDR } 0.60\end{array}$ \\
\hline $\begin{array}{l}\text { Marriott et al. } \\
(2008)^{(9)} \text { UK (3.5) }\end{array}$ & $\begin{array}{l}n 50 \\
12 \text { months } \\
\text { Infants }\end{array}$ & $\begin{array}{l}\text { FFQ v. } 4 \text { d WDR } \\
\text { seventy-eight items. } \\
\text { HH measures }\end{array}$ & $\begin{array}{l}\text { Energy adjusted } \\
\text { CC } 0.54\end{array}$ & $\begin{array}{l}\text { Median }(\mu \mathrm{g}) \\
\text { FFQ } 1.09 \\
\text { WDR } 0.81\end{array}$ \\
\hline $\begin{array}{l}\text { Blum et al. } \\
(1999)^{(24)} \text { USA (2.5) }\end{array}$ & $\begin{array}{l}n 233 \\
1-5 \text { years } \\
\text { Infants } \\
\text { Preschool children }\end{array}$ & $\begin{array}{l}\text { FFQ v. three } 24 \mathrm{HR} \\
\text { eighty-four items. }\end{array}$ & CC 0.58 & $\begin{array}{l}\text { Mean (SD) (mg) } \\
24 \text { HR } 2(1) \\
\text { FFQ } 2(1)\end{array}$ \\
\hline $\begin{array}{l}\text { Bellù et al. } \\
(1995)^{(32)} \text { Italy (3.5) }\end{array}$ & $\begin{array}{l}n 88 \\
9-12 \text { years } \\
\text { Children }\end{array}$ & $\begin{array}{l}\text { FFQ } v .7 \mathrm{~d} \text { EDR } \\
116 \text { items. Reference } \\
\text { portion sizes }\end{array}$ & $\begin{array}{l}\text { Raw nutrient } 0.17 \\
\text { Nutrient density } 0.26\end{array}$ & $\begin{array}{l}\text { Mean (SD) } \\
\text { EDR } 0.28(0.09) \\
\text { FFQ } 0.25(0.1)\end{array}$ \\
\hline $\begin{array}{l}\text { Rockett et al. } \\
(1997)^{(29)} \text { USA (3.5) }\end{array}$ & $\begin{array}{l}\text { n } 261 \\
9-18 \text { years } \\
\text { Children Adolescents }\end{array}$ & $\begin{array}{l}\text { YAQ v. 3-24 HR } \\
131 \text { items. Standard } \\
\text { reference portions }\end{array}$ & $\begin{array}{l}9-13 \text { years CC } 0.51 \\
14-18 \text { years CC } 0.55\end{array}$ & $\begin{array}{l}\text { Mean }(\mathrm{SD})(\mathrm{mg}) \\
24 \mathrm{HR} 2 \cdot 2(1.5) \\
\text { YAQ } 2 \cdot 2(1 \cdot 0)\end{array}$ \\
\hline \multicolumn{5}{|l|}{ Vitamin $\mathrm{B}_{12}$} \\
\hline $\begin{array}{l}\text { Marriott et al. } \\
(2008)^{(8)} \text { UK (3.5) }\end{array}$ & $\begin{array}{l}n 50 \\
6 \text { months } \\
\text { Infants }\end{array}$ & $\begin{array}{l}\text { FFQ v. } 4 \mathrm{~d} \text { WDR } \\
\text { thirty-four items. } \mathrm{HH} \\
\text { measures }\end{array}$ & $\begin{array}{l}\text { Energy adjusted } \\
\text { CC } 0.74\end{array}$ & $\begin{array}{l}\text { Median }(\mu \mathrm{g}) \\
\text { FFQ } 1.8 \\
\text { WDR } 1.8\end{array}$ \\
\hline $\begin{array}{l}\text { Marriott et al. } \\
(2008)^{(9)} \text { UK (3.5) }\end{array}$ & $\begin{array}{l}n 50 \\
12 \text { months } \\
\text { Infants }\end{array}$ & $\begin{array}{l}\text { FFQ v. } 4 \text { d WDR } \\
\text { seventy-eight items. } \\
\text { HH measures }\end{array}$ & $\begin{array}{l}\text { Energy adjusted } \\
\text { CC } 0.24\end{array}$ & $\begin{array}{l}\text { Median }(\mu \mathrm{g}) \\
\text { FFQ } 3.08 \\
\text { WDR } 2.8\end{array}$ \\
\hline $\begin{array}{l}\text { Blum et al. } \\
(1999)^{(24)} \text { USA (2.5) }\end{array}$ & $\begin{array}{l}n 233 \\
1-5 \text { years } \\
\text { Infants } \\
\text { Preschool children }\end{array}$ & $\begin{array}{l}\text { FFQ v. 3-24 HR } \\
\text { eighty-four items. }\end{array}$ & $\mathrm{CC} 0.47$ & $\begin{array}{l}\text { Mean (SD) (mg) } \\
24 \text { HR } 5 \text { (3) } \\
\text { FFQ } 6 \text { (2) }\end{array}$ \\
\hline $\begin{array}{l}\text { Rockett et al. } \\
(1997)^{(29)} \text { USA (3.5) }\end{array}$ & $\begin{array}{l}\text { n } 261 \\
9-18 \text { years } \\
\text { Children } \\
\text { Adolescents }\end{array}$ & $\begin{array}{r}\text { YAQ } v . \text { three } 24 \mathrm{HR} \\
131 \text { items. Standard } \\
\text { reference portions }\end{array}$ & $\begin{array}{l}9-13 \text { years CC } 0.40 \\
14-18 \text { years CC } 0.38\end{array}$ & $\begin{array}{l}\text { Mean }(\mathrm{SD})(\mu \mathrm{g}) \\
24 \mathrm{HR} 5.7(4.7) \\
\text { YAQ } 7.6(3.8)\end{array}$ \\
\hline Green et al. & $n 105$ & FFQ $v .3 d$ WDR & Food only & Food + supplements \\
\hline
\end{tabular}


Author/year

publication/country/

(quality index)

$(1998)^{(28)}$ Canada

$(4.5)$

Folate

Blum et al.

(1999) $^{(24)}$ USA (2.5)

n 233

$1-5$ years

Infants

Rockett et al.

$(1997)^{(29)}$ USA (3.5)

reschool children

9-18 years

Children

Adolescents

Green et al.

$(1998)^{(28)}$ Canada

(4.5)

$n 105$

16-19 years

Adolescents

\section{Vitamin C}

Marriott et al

$(2008)^{(8)}$ UK (3.5)

$n 50$

6 months

Infants

Marriott et al

(2008) $)^{(9)}$ UK (3.5)

$n 50$

12 months

Infants

Williams \& Innis

$(2005)^{(17)}$ Canada

(5)

Andersen et al.

$(2003)^{(2)}$ Norway

(4.5)

8-26 months

Infants

$n 64$

12 months

Infants

Blum et al.

(1999) ${ }^{(24)}$ USA (2.5)

$n 233$

$1-5$ years

Infants

Preschool children

Parrish et al.

$(2003)^{(22)}$ USA (3.5)

$n 38$

$1-3$ years

Infants

Preschool children

Andersen et al.

$(2004)^{(18,19)}$ Norway

(5)

$n 187$

2 years old

Preschool children

Holmes et al.

$(2008)^{(10)}$ UK (3)

n 124

2-17 years
Methods/

no FFQ items,

portion sizes

Correlation between methods/other statistics

116 items. $\mathrm{HH}$

measures

Median (1st - 3rd quartile) $(\mu \mathrm{g})$

Median (1st - 3rd quartile) $(\mu)$

FFQ $4.4(3.4,7.0)$

FFQ $v$. BM

3d WDR $v$. BM

WDR $1.7(0.7,2.4)$

CC 0.19

FFQ v. three $24 \mathrm{HR}$

CC 0.55

eighty-four items.

YAQ v. three $24 \mathrm{HR}$

131 items. Standard

reference portions

$9-13$ years $\mathrm{CC} 0.53$

$14-18$ years $\mathrm{CC} 0.63$

FFQ v. 3d WDR

116 items. $\mathrm{HH}$

measures

FFQ $v$. BM

$3 \mathrm{~d}$ WDR $v$. BM

FFQ v. 4 d WDR

thirty-four items. $\mathrm{HH}$

measures

FFQ v. 4 d WDR

seventy-eight items.

$\mathrm{HH}$ measures

FFQ $v .3 \mathrm{~d}$ EDR

191 items

Food only

Food + supplements

FFQ $289(218,400)$

WDR $187(0.7,2.4)$

CC 0.38

CC 0.46

Energy adjusted

CC 0.67

Energy adjusted

CC 0.47

CC 0.64

FFQ $v .7 \mathrm{~d}$ WDR

140 items. $\mathrm{HH}$

measures or food

photographs

FFQ v. three $24 \mathrm{HR}$

eighty-four items.

FFQ $v$. BM

111 items. Standard

reference portions

$24 \mathrm{HR} v$. BM

FFQ $v .7$ d WDR

125 items. $\mathrm{HH}$

measures or food

photographs

$24 \mathrm{HR}$ v. 4d WDR
Absolute intake

CC 0.41

CC 0.58

CC 0.51

CC 0.16

Absolute intake

CC $0.43^{\star \star \star}$

Males-Mean difference

2-10 years $16(P=0.042)$
Median (1st-3rd quartile) $(\mu \mathrm{g})$

FFQ $4.9(3.6,10)$

WDR $1.9(1.0,3.7)$

CC 0.25

Mean (SD) $(\mu \mathrm{g})$

24 HR 274 (133)

FFQ 307 (147)

Mean (SD) ( $\mu \mathrm{g})$

24 HR 373 (216)

YAQ 382 (187)

FFQ $346(229,494)$

WDR $212(155,301)$

CC 0.48

CC 0.65

Median (mg)

FFQ 86

WDR 78

Median $(\mu \mathrm{g})$

FFQ 79.6

WDR $42 \cdot 3$

Median (mg)

FFQ 74

Median $\left(\mathrm{P}_{25}-\mathrm{P}_{75}\right)(\mathrm{mg})$

FFQ 88 (55-118)

WDR $51(33-77)^{\star \star *}$

Mean (SD) (mg)

24 HR 117 (66)

FFQ 114 (49)

Median $\left(\mathrm{P}_{25}-\mathrm{P}_{75}\right)(\mathrm{mg})$

FFQ $88(62-119)$

WDR $60(42-79)^{\star \star \star}$

Females-Mean difference

$2-10$ years $28(P=0.009)$ 


\begin{tabular}{|c|c|c|c|c|}
\hline \multirow[t]{2}{*}{$\begin{array}{l}\text { Author/year } \\
\text { publication/country/ } \\
\text { (quality index) }\end{array}$} & \multirow{2}{*}{$\begin{array}{l}\text { n/age group } \\
\text { Preschool children } \\
\text { Children } \\
\text { Adolescents }\end{array}$} & \multirow[t]{2}{*}{$\begin{array}{l}\text { Methods/ } \\
\text { no FFQ items, } \\
\text { portion sizes }\end{array}$} & \multicolumn{2}{|c|}{ Correlation between methods/other statistics } \\
\hline & & & $11-17$ years $4(P=0.656)$ & $11-17$ years $15(P=0.207)$ \\
\hline & & FC v. $4 \mathrm{~d}$ WDR & $\begin{array}{l}\text { Males-Mean difference } \\
2-10 \text { years } 33(P=0.002) \\
11-17 \text { years } 18(P=0.231)\end{array}$ & $\begin{array}{l}\text { Females-Mean difference } \\
2-10 \text { years } 43(P=0.001) \\
11-17 \text { years } 24(P=0.325)\end{array}$ \\
\hline & & SW v. $4 \mathrm{~d}$ WDR & $\begin{array}{l}\text { Males-Mean difference } \\
2-10 \text { years } 6(P=0.472) \\
11-17 \text { years }-7(P=0.676)\end{array}$ & $\begin{array}{l}\text { Females-Mean difference } \\
2-10 \text { years } 7(P=0.639) \\
11-17 \text { years }-31(P=0.017)\end{array}$ \\
\hline $\begin{array}{l}\text { Räsänen L. } \\
(1979)^{(39)} \text { Finland } \\
(5)\end{array}$ & $\begin{array}{l}n 741 \\
5-13 \text { years } \\
\text { Preschool children } \\
\text { Children } \\
\text { Adolescents }\end{array}$ & $24 \mathrm{HR} v . \mathrm{DH}$ & $\mathrm{CC} 0.21$ & Mean DH: 113; 24 HR: 85 \\
\hline $\begin{array}{l}\text { Byers et al. } \\
(1993)^{(35)} \text { USA }(2 \cdot 5)\end{array}$ & $\begin{array}{l}n 97 \\
6-10 \text { years } \\
\text { Children }\end{array}$ & $\begin{array}{l}\mathrm{FFQ} v . \mathrm{BM} \\
111 \text { items }\end{array}$ & $\begin{array}{l}\text { Crude } 0.39 \\
\text { Adjusted } 0.37\end{array}$ & \\
\hline $\begin{array}{l}\text { Arnold et al. } \\
(1995)^{(31)} \text { Canada } \\
(3.5)\end{array}$ & $\begin{array}{l}n 707 \\
7-12 \text { years } \\
\text { Children }\end{array}$ & $\begin{array}{l}\text { FFQ } v .14 \mathrm{~d} \text { EDR } \\
160 \text { items. Standard } \\
\text { reference portions }\end{array}$ & Pearson CC $0.57 \dagger$ & \\
\hline $\begin{array}{l}\text { Lillegaard et al. } \\
(2007)^{(12)} \text { Norway }\end{array}$ & $\begin{array}{l}n 100 \\
9 \text { years old }\end{array}$ & $\begin{array}{l}\text { PFD v. } 4 \text { d WDR } \\
277 \text { items. HH }\end{array}$ & $\begin{array}{l}\text { Boys-Median }\left(\mathrm{P}_{25-} \mathrm{P}_{75}\right)(\mathrm{mg}) \\
\text { PFD } 86(53,123)\end{array}$ & $\begin{array}{l}\text { Girls-Median }\left(\mathrm{P}_{25-} \mathrm{P}_{75}\right)(\mathrm{mg}) \\
\text { PFD } 87(66,104)\end{array}$ \\
\hline (3.5) & Children & $\begin{array}{l}\text { measures or colour } \\
\text { photographs }\end{array}$ & $\begin{array}{l}\text { WDR } 80(54,113) \\
\text { CC } 0.40^{* *}\end{array}$ & $\begin{array}{l}\text { WDR } 63(38,96) \\
\text { CC } 0.42^{\star \star *}\end{array}$ \\
\hline Bellù et al. & $n 88$ & FFQ v.7d EDR & Raw nutrient 0.45 & Mean (SD) \\
\hline$(1995)^{(32)}$ Italy (3.5) & $\begin{array}{l}9-12 \text { years } \\
\text { Children }\end{array}$ & $\begin{array}{l}116 \text { items. Reference } \\
\text { portion sizes }\end{array}$ & Nutrient density 0.53 & $\begin{array}{l}\text { EDR } 34.6(22 \cdot 2) \\
\text { FFQ } 52 \cdot 6(26 \cdot 7)\end{array}$ \\
\hline $\begin{array}{l}\text { Field et al. } \\
(1998)^{(25)} \text { USA (4) }\end{array}$ & $\begin{array}{l}n 109 \\
\text { Students from fourth } \\
\text { to seventh grade } \\
\text { Children }\end{array}$ & $\begin{array}{l}\mathrm{FFQ} v \text {. four } 24 \mathrm{HR} \\
\text { ninety-seven items. } \\
\text { Standard reference } \\
\text { portions. }\end{array}$ & $\begin{array}{l}\text { Fourth and fifth grades } \\
\text { Crude } 0 \cdot 19 \\
\text { Deattenuated } 0.32\end{array}$ & $\begin{array}{l}\text { Sixth and seventh grades } \\
\text { Crude } 0.50 \\
\text { Deattenuated } 0.76\end{array}$ \\
\hline $\begin{array}{l}\text { Rockett et al. } \\
(1997)^{(29)} \text { USA (3.5) }\end{array}$ & $\begin{array}{l}n 261 \\
9-18 \text { years } \\
\text { Children } \\
\text { Adolescents }\end{array}$ & $\begin{array}{r}\text { YAQ } v \text {. three } 24 \mathrm{HR} \\
131 \text { items. Standard } \\
\text { reference portions }\end{array}$ & $\begin{array}{l}9-13 \text { years } C C 0.61 \\
14-18 \text { years } C C 0.45\end{array}$ & $\begin{array}{l}\text { Mean }(\mathrm{sD})(\mathrm{mg}) \\
24 \mathrm{HR} 166(164) \\
\text { YAQ } 161(80)\end{array}$ \\
\hline $\begin{array}{l}\text { Slater et al. } \\
(2003)^{(20)} \text { Brazil (4) }\end{array}$ & $\begin{array}{l}n 79 \\
14-18 \text { years } \\
\text { Adolescents }\end{array}$ & $\begin{array}{l}\text { FFQ } v \text {. three } 24 \mathrm{HR} \\
\text { seventy-six items. } \\
\text { Reference portion } \\
\text { size }\end{array}$ & $\begin{array}{l}\text { Non-adjusted } 0.42^{\star \star} \\
\text { Energy adjusted } 0.47^{\star \star} \\
\text { Deattenuated } 0.91\end{array}$ & $\begin{array}{l}\text { Mean }(\mathrm{SD})(\mathrm{mg}) \\
24 \mathrm{HR} 69.8(86.5) \\
\text { FFQ } 79.9(41.6)\end{array}$ \\
\hline $\begin{array}{l}\text { Andersen et al. } \\
(1995)^{(33)} \text { Norway } \\
\text { (3) }\end{array}$ & $\begin{array}{l}n 49 \\
\text { Adolescents }\end{array}$ & $\begin{array}{l}\text { FFQ } v .7 \mathrm{~d} \text { WDR } \\
190 \text { items. HH } \\
\text { measures }\end{array}$ & $\begin{array}{l}\text { Unadjusted } 0.34 \\
\text { Energy adjusted } 0.37\end{array}$ & $\begin{array}{l}\text { Median FFQ: } 123(\mathrm{mg}) \\
\text { Median WDR: } 72(\mathrm{mg})\end{array}$ \\
\hline
\end{tabular}




\begin{tabular}{|c|c|c|c|c|}
\hline $\begin{array}{l}\text { Author/year } \\
\text { publication/country/ } \\
\text { (quality index) }\end{array}$ & n/age group & $\begin{array}{l}\text { Methods/ } \\
\text { no FFQ items, } \\
\text { portion sizes }\end{array}$ & Correlation between methods/other statistics & \\
\hline \multicolumn{5}{|l|}{$\mathrm{Na}$} \\
\hline $\begin{array}{l}\text { Marriott et al. } \\
(2008)^{(8)} \text { UK (3.5) }\end{array}$ & $\begin{array}{l}n 50 \\
6 \text { months } \\
\text { Infants }\end{array}$ & $\begin{array}{l}\text { FFQ } v .4 \mathrm{~d} \text { WDR } \\
\text { thirty-four items } \mathrm{HH} \\
\text { measures }\end{array}$ & $\begin{array}{l}\text { Energy adjusted } \\
\text { CC } 0.56\end{array}$ & $\begin{array}{l}\text { Median }(\mathrm{mg}) \\
\text { FFQ } 248 \\
\text { WDR } 270\end{array}$ \\
\hline $\begin{array}{l}\text { Marriott et al. } \\
(2008)^{(9)} \text { UK (3.5) }\end{array}$ & $\begin{array}{l}n 50 \\
12 \text { months } \\
\text { Infants }\end{array}$ & $\begin{array}{l}\text { FFQ v. } 4 \text { d WDR } \\
\text { seventy-eight items } \\
\text { HH measures }\end{array}$ & $\begin{array}{l}\text { Energy adjusted } \\
\text { CC } 0.75\end{array}$ & $\begin{array}{l}\text { Median }(\mu \mathrm{g}) \\
\text { FFQ } 861 \\
\text { WDR } 862\end{array}$ \\
\hline $\begin{array}{l}\text { lannotti et al. } \\
(1994)^{(34)} \text { USA (3) }\end{array}$ & $\begin{array}{l}n 17 \\
2-4 \text { years } \\
\text { Preschool children }\end{array}$ & FFQ $v$. three $24 \mathrm{HR}$ & CC 0.27 & $\begin{array}{l}\text { Mean (SD) } \\
\text { FFQ } 1307(639) \\
24 \text { HR } 1410(656)\end{array}$ \\
\hline $\begin{array}{l}\text { Stein et al. (1992) } \\
\text { USA (5.5) }\end{array}$ & $\begin{array}{l}\text { n } 224 \\
3.8-5 \text { years } \\
\text { Preschool children }\end{array}$ & FFQ $v$. four $24 \mathrm{HR}$ & $\begin{array}{l}\text { Boys } \\
\text { Absolute intake } 0.22 \\
\text { Adjusted for energy } 0.04 \\
\text { Adjusted for energy and } \\
\text { intra-individual variability } 0.06 \\
\text { Mean (SD) } \\
24 \text { HR } 1895 \text { (584) } \\
\text { FFQ } 2187 \text { (592) }\end{array}$ & $\begin{array}{l}\text { Girls } \\
\text { Absolute intake } 0.30 \\
\text { Adjusted for energy }-0.09 \\
\text { Adjusted for energy and } \\
\text { intra-individual } \\
\quad \text { variability }-0.14 \\
\text { Mean (SD) } \\
24 \text { HR } 1860(675) \\
\text { FFQ 2120 (654) }\end{array}$ \\
\hline $\begin{array}{l}\text { Lytle et al. }(1993)^{(36)} \\
\text { USA (3.5) }\end{array}$ & $\begin{array}{l}n 49 \\
8 \text { years old } \\
\text { Children }\end{array}$ & $24 \mathrm{HR} v .1 \mathrm{~d}$ EDR & CC 0.53 & $\begin{array}{l}\text { Mean (sD) } \\
\text { EDR } 2554 \text { mg (969) } \\
24 \text { HR } 2524 \text { mg (1089) }\end{array}$ \\
\hline $\begin{array}{l}\text { Rockett et al. } \\
(1997)^{(29)} \text { USA (3.5) }\end{array}$ & $\begin{array}{l}n 261 \\
9-18 \text { years } \\
\text { Children } \\
\text { Adolescents }\end{array}$ & $\begin{array}{l}\text { YAQ v. three } 24 \mathrm{HR} \\
131 \text { items. Standard } \\
\text { reference portions }\end{array}$ & $\begin{array}{l}9-13 \text { years CC } 0.13 \\
14-18 \text { years CC } 0.32\end{array}$ & $\begin{array}{l}\text { Mean }(\mathrm{sD})(\mathrm{mg}) \\
24 \text { HR } 2393(1120) \\
\text { YAQ } 2753(718)\end{array}$ \\
\hline Lietz et al. (2002) ${ }^{(23)}$ & $n 50$ & FFQ v. 7d WDR & Unadjusted 0.29 & Mean (SD) (mg) \\
\hline UK (3.5) & $\begin{array}{l}11-13 \text { years } \\
\text { Children }\end{array}$ & $\begin{array}{r}130 \text { items. Standard } \\
\text { reference portions }\end{array}$ & Energy adjusted 0.26 & $\begin{array}{l}\text { WDR } 2634 \text { (522) } \\
\text { FFQ } 3768 \text { (1341) }\end{array}$ \\
\hline \multicolumn{5}{|c|}{ 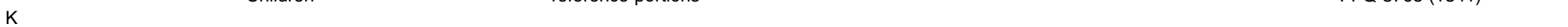 } \\
\hline $\begin{array}{l}\text { Marriott et al. } \\
(2008)^{(8)} \text { UK (3.5) }\end{array}$ & $\begin{array}{l}n 50 \\
6 \text { months } \\
\text { Infants }\end{array}$ & $\begin{array}{l}\text { FFQ } v .4 \mathrm{~d} \text { WDR } \\
\text { thirty-four items } \\
\text { Quantified, } \\
\text { HH measures }\end{array}$ & $\begin{array}{l}\text { Energy adjusted } \\
\text { CC } 0.70\end{array}$ & $\begin{array}{l}\text { Median }(\mathrm{mg}) \\
\text { FFQ } 1035 \\
\text { WDR } 1014\end{array}$ \\
\hline $\begin{array}{l}\text { Marriott et al. } \\
(2008)^{(9)} \text { UK (3.5) }\end{array}$ & $\begin{array}{l}n 50 \\
12 \text { months } \\
\text { Infants }\end{array}$ & $\begin{array}{l}\text { FFQ } v .4 \mathrm{~d} \text { WDR } \\
\text { seventy-eight items } \\
\text { HH measures }\end{array}$ & $\begin{array}{l}\text { Energy adjusted } \\
\text { CC } 0.42\end{array}$ & $\begin{array}{l}\text { Median }(\mu \mathrm{g}) \\
\text { FFQ } 1744 \\
\text { WDR } 1402\end{array}$ \\
\hline Stein et al. $(1992)^{(37)}$ & $n 224$ & FFQ v. four $24 \mathrm{HR}$ & Boys & Girls \\
\hline USA $(5 \cdot 5)$ & $\begin{array}{l}3.8-5 \text { years } \\
\text { Preschool children }\end{array}$ & & $\begin{array}{l}\text { Absolute intake } 0.33 \\
\text { Adjusted for energy } 0.57 \\
\text { Adjusted for energy and } \\
\text { intra-individual variability } 0.71 \\
\text { Mean (SD) } \\
24 \text { HR } 2360 \text { (609) } \\
\text { FFQ } 3860(953)\end{array}$ & $\begin{array}{l}\text { Absolute intake } 0.60 \\
\text { Adjusted for energy } 0.62 \\
\text { Adjusted for energy and } \\
\text { intra-individual } \\
\text { variability } 0.78 \\
\text { Mean (SD) } \\
24 \mathrm{HR} 2190^{*}(549) \\
\text { FFQ } 3722 \text { (973) }\end{array}$ \\
\hline Jenner et al. & $n 225$ & FFQ $v$. fourteen $24 \mathrm{HR}$ & FFQ completed by children CC 0.13 & Mean (SD) \\
\hline $\begin{array}{l}(1989)^{(38)} \text { Australia } \\
(3.5)\end{array}$ & $\begin{array}{l}11-12 \text { years } \\
\text { Children }\end{array}$ & 175 items & FFQ completed by parents CC 0.32 & $\begin{array}{l}24 \mathrm{HR} 2.6(0.7) \\
\text { FFQ (children) } 3.3(1.6) \\
\text { FFQ (parents) } 2.9(0.8)\end{array}$ \\
\hline $\begin{array}{l}\text { Rockett et al. } \\
(1997)^{(29)} \text { USA (3.5) }\end{array}$ & $\begin{array}{l}n 261 \\
9-18 \text { years }\end{array}$ & $\begin{array}{l}\text { YAQ v. three } 24 \mathrm{HR} \\
131 \text { items. }\end{array}$ & $\begin{array}{l}9-13 \text { years } C C \text { C } 0.41 \\
14-18 \text { years } C C \quad 0.48\end{array}$ & $\begin{array}{l}\text { Mean (SD) (mg) } \\
24 \text { HR } 2608(956)\end{array}$ \\
\hline
\end{tabular}


Table 4. Continued

\begin{tabular}{|c|c|c|c|c|c|c|}
\hline $\begin{array}{l}\text { Author/year } \\
\text { publication/country/ } \\
\text { (quality index) }\end{array}$ & n/age group & $\begin{array}{l}\text { Methods/ } \\
\text { no FFQ items, } \\
\text { portion sizes }\end{array}$ & \multicolumn{4}{|c|}{ Correlation between methods/other statistics } \\
\hline & $\begin{array}{l}\text { Children } \\
\text { Adolescents }\end{array}$ & Standard reference portions & & & YAQ 2772 (859) & \\
\hline Lietz et al. (2002) & $n 50$ & FFQ v. $7 \mathrm{~d}$ WDR & Unadjusted 0.27 & & Mean (SD) & \\
\hline \multirow{2}{*}{ UK (3.5) } & $11-13$ years & 130 items. & Energy adjusted $0 \cdot 60^{\star *}$ & & WDR 2347 (619) & \\
\hline & Children & $\begin{array}{l}\text { Standard reference portions } \\
7 \mathrm{~d} \text { WDR } v . \mathrm{BM} \\
\text { FFQ v. BM }\end{array}$ & $\begin{array}{l}\text { CC } 0.78^{\star \star \star} \\
\text { CC } 0.04\end{array}$ & & FFQ 3660 (1288) & \\
\hline \multicolumn{7}{|c|}{ 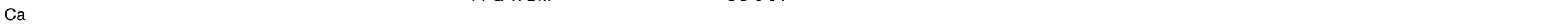 } \\
\hline $\begin{array}{l}\text { Marriott et al. } \\
(2008)^{(8)} \text { UK (3.5) }\end{array}$ & $\begin{array}{l}n 50 \\
6 \text { months } \\
\text { Infants }\end{array}$ & $\begin{array}{l}\text { FFQ } v .4 d \text { WDR } \\
\text { thirty-four items } \\
\text { HH measures }\end{array}$ & $\begin{array}{l}\text { Energy adjusted } \\
\text { CC } 0.78\end{array}$ & & $\begin{array}{l}\text { Median (mg) } \\
\text { FFQ } 547 \\
\text { WDR } 543\end{array}$ & \\
\hline $\begin{array}{l}\text { Marriott et al. } \\
(2008)^{(9)} \text { UK (3.5) }\end{array}$ & $\begin{array}{l}n 50 \\
12 \text { months } \\
\text { Infants }\end{array}$ & $\begin{array}{l}\text { FFQ } v .4 \text { d WDR } \\
\text { seventy-eight items } \\
\text { HH measures }\end{array}$ & $\begin{array}{l}\text { Energy adjusted } \\
\text { CC } 0.61\end{array}$ & & $\begin{array}{l}\text { Median }(\mu \mathrm{g}) \\
\text { FFQ } 877 \\
\text { WDR } 784\end{array}$ & \\
\hline $\begin{array}{l}\text { Williams \& Innis } \\
(2005)^{(17)} \text { Canada } \\
(5)\end{array}$ & $\begin{array}{l}n 148 \\
8-26 \text { months } \\
\text { Infants }\end{array}$ & $\begin{array}{l}\text { FFQ } v .3 d \text { EDR } \\
191 \text { items }\end{array}$ & CC 0.75 & & $\begin{array}{l}\text { Median }(\mathrm{mg}) \\
\text { FFQ } 861 \\
\text { EDR } 691\end{array}$ & \\
\hline $\begin{array}{l}\text { Andersen et al. } \\
(2003)^{(2)} \text { Norway } \\
(4.5)\end{array}$ & $\begin{array}{l}n 64 \\
12 \text { months } \\
\text { Infants }\end{array}$ & $\begin{array}{l}\text { FFQ v. } 7 \mathrm{~d} \text { WDR } \\
140 \text { items. HH measures or } \\
\text { food photographs }\end{array}$ & $\begin{array}{l}\text { Absolute intake } \\
\text { CC } 0.62\end{array}$ & & $\begin{array}{l}\text { Median }\left(\mathrm{P}_{25}-\mathrm{P}_{75}\right)(\mathrm{mg}) \\
\text { FFQ } 584(377-773) \\
\text { WDR } 527(329-734)\end{array}$ & \\
\hline \multirow{4}{*}{$\begin{array}{l}\text { Marshall et al. } \\
(2003)^{(21)} \text { USA (4) }\end{array}$} & $n 240$ & FFQ v. 3d WDR & 6 months & 12 months & 3 years & 5 years \\
\hline & $6-12$ months & seven items. Estimated & CC $0.64^{\star * *}$ & $\operatorname{CC} 0.67^{* \star *}$ & $\operatorname{CC} 0 \cdot 74^{\star \star \star}$ & $\operatorname{CC} 0 \cdot 64^{* * *}$ \\
\hline & $\begin{array}{l}3-5 \text { years } \\
\text { Infants }\end{array}$ & usual serving size & $\begin{array}{l}\text { Mean (SD) mg } \\
\text { FFQ } 409(153)\end{array}$ & $\begin{array}{l}\text { Mean (SD) mg } \\
\text { FFQ } 608(378)\end{array}$ & $\begin{array}{l}\text { Mean (SD) mg } \\
\text { FFQ } 383(269)\end{array}$ & $\begin{array}{l}\text { Mean (SD) mg } \\
\text { FFQ } 393(245)\end{array}$ \\
\hline & Preschool children & & WDR $380(137)$ & WDR 601(340) & WDR 449(269) & WDR 406 (222) \\
\hline \multirow{2}{*}{$\begin{array}{l}\text { Blum et al. }(1999)^{(24)} \\
\text { USA (2.5) }\end{array}$} & n 233 & FFQ $v$. three $24 \mathrm{HR}$ & CC 0.60 & & Mean (SD) (mg) & \\
\hline & $\begin{array}{l}1-5 \text { years } \\
\text { Infants } \\
\text { Preschool children }\end{array}$ & eighty-four items & & & $\begin{array}{l}24 \mathrm{HR} 1016(328) \\
\text { FFQ } 1087(319)\end{array}$ & \\
\hline \multirow{10}{*}{$\begin{array}{l}\text { Andersen et al. } \\
(2004)^{(18,19)} \text { Norway } \\
(5) \\
\text { Huybrechts et al. } \\
(2006)^{(15)} \text { Belgium } \\
(3 \cdot 5) \\
\text { Stein et al. }(1992)^{(37)} \\
\text { USA (5.5) }\end{array}$} & $\begin{array}{l}n 187 \\
2 \text { years old } \\
\text { Preschool children }\end{array}$ & $\begin{array}{l}\text { FFQ } v .7 \mathrm{~d} \text { WDR } \\
125 \text { items. HH measures } \\
\text { or food photographs }\end{array}$ & $\begin{array}{l}\text { Absolute intake } \\
\text { CC } 0.38^{\star * \star}\end{array}$ & & $\begin{array}{l}\text { Median }\left(\mathrm{P}_{25}-\mathrm{P}_{75}\right)(\mathrm{mg}) \\
\text { FFQ } 689(559-872) \\
\text { WDR } 520(106-649)^{\star \star *}\end{array}$ & \\
\hline & $\begin{array}{l}n 509 \\
2 \cdot 5-6.5 \text { years }\end{array}$ & $\begin{array}{l}\text { FFQ v. 3d EDR } \\
\text { forty-seven items. Portion }\end{array}$ & CC 0.52 & & $\begin{array}{l}\text { Mean (sD) } \\
\text { EDR } 838(305)\end{array}$ & \\
\hline & Preschool children & size alternatives included. & & & FFQ 777 (296) & \\
\hline & $n 224$ & FFQ $v$. four $24 \mathrm{HR}$ & Boys & & Girls & \\
\hline & $3.8-5$ years & & Absolute intake 0.50 & & Absolute intake 0.50 & \\
\hline & Preschool children & & Adjusted for energy 0.54 & & Adjusted for energy 0.47 & \\
\hline & & & $\begin{array}{l}\text { Adjusted for energy and } \\
\text { intra-individual variability } 0.65\end{array}$ & & $\begin{array}{l}\text { Adjusted for energy and } \\
\text { intra-individual }\end{array}$ & \\
\hline & & & Mean (SD) & & variability 0.57 & \\
\hline & & & 24 HR 880 (283) & & Mean (SD) & \\
\hline & & & FFQ 1418 (404) & & $\begin{array}{l}24 \mathrm{HR} 816(248) \\
\text { FFQ } 1351(369)\end{array}$ & \\
\hline \multirow{6}{*}{$\begin{array}{l}\text { Holmes et al. } \\
(2008)^{(10)} \text { UK (3) }\end{array}$} & $n 124$ & $24 \mathrm{HR} v .4 \mathrm{~d}$ WDR & Males-Mean difference & & Females-Mean difference & \\
\hline & $2-17$ years & & $2-10$ years $15(P=0.751)$ & & $2-10$ years $133(P=0.001)$ & \\
\hline & $\begin{array}{l}\text { Preschool children } \\
\text { Children }\end{array}$ & & $11-17$ years $142(P=0.012)$ & & $11-17$ years $39(P=0.522)$ & \\
\hline & Adolescents & & & & & \\
\hline & & FC v. $4 \mathrm{~d}$ WDR & Males-Mean difference & & Females-Mean difference & \\
\hline & & & $11-17$ years $39(P=0.480)$ & & $11-17$ years $77(P=0.205)$ & \\
\hline
\end{tabular}




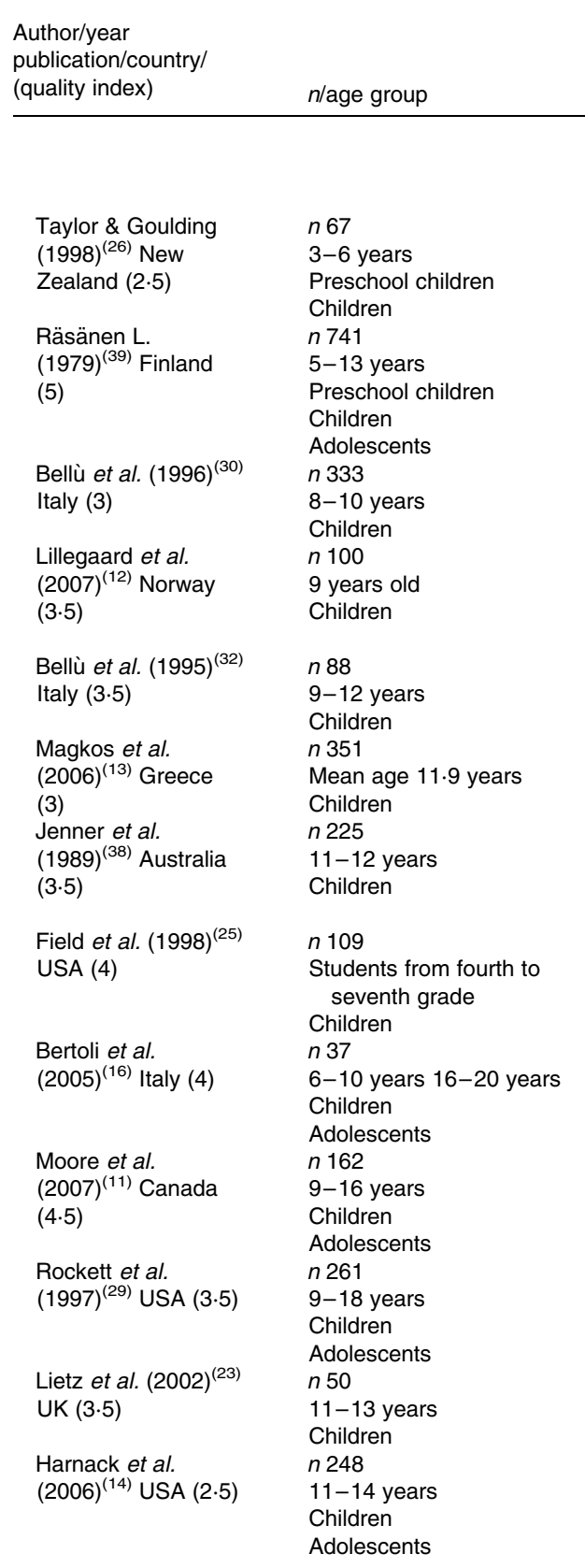

Methods/

portion sizes

Correlation between methods/other statistics

SW v. 4 d WDR

Males-Mean difference

-10 years $-77(P=0.245)$

FFQ $v .4 d E D R$

CC 0.52

thirty-five items.

$24 \mathrm{HR} v$. DH

CC 0.47

FFQ $v .24 \mathrm{HR}$

116 items.

PFD v. 4 d WDR

277 items. $\mathrm{HH}$ measures

or colour photographs

FFQ v. 7d EDR

116 items. Reference

portion sizes

thirty items. Standard

175 items

FFQ $v$, four $24 \mathrm{HR}$

ninety-seven items.

Standard reference

portions used.

FFQ $v .7 \mathrm{~d}$ WDR

136 items Portion size

photographs

FFQ v. $24 \mathrm{HR}$

thirty-two items. Visual aids

to approximate portion

size

YAQ $v$. three $24 \mathrm{HR}$

131 items. Standard

reference portions

FFQ $v .7 \mathrm{~d}$ WDR

130 items. Standard

reference portion

FFQ $v$. three $24 \mathrm{HR}$

ten items. Portion size

alternatives included
Girls Mean (SD)

$24 \mathrm{HR} 441$ (179)

FFQ 445 (125)

Boys-Median $\left(\mathrm{P}_{25}-\mathrm{P}_{75}\right)(\mathrm{mg})$

PFD 715 (581-1036)

WDR 712 (578-917)

CC $0.49^{\star \star \star}$

Raw nutrient 0.32

Nutrient density 0.42

CC $0.63^{\star \star *}$

FFQ completed by

children CC 0.20

FFQ completed by

parents CC 0.31

Fourth and fifth

grades Crude 0.27

Young adults $\mathrm{CC} 0.5$

Children CC 0.60

9-12 years ICC 0.46

14-16 years ICC 0.43

9-13 years CC 0.52

$14-18$ years CC 0.57

Unadjusted $0.34^{*}$

Energy adjusted $0.47^{\star *}$

$11-12$ years ICC 0.59

13-14 years ICC 0.33
Females-Mean difference

$2-10$ years $38(P=0.452)$
$11-17$ years $43(P=0.613)$

Mean (SD)

EDR $798 \mathrm{mg}(271)$

FFQ $942 \mathrm{mg}$ (419)

Mean DH: 1369; 24 HR: 103

Boys Mean (SD)

24 HR 453 (203)

FFQ 435 (140)

Girls-Median $\left(\mathrm{P}_{25} \mathrm{P}_{75}\right)(\mathrm{mg})$

PFD 740 (572-962)

WDR 630 (473-877)

CC $0.56^{\star \star *}$

(M)

EDR $377(106)$

EDR $377(106)$

FFQ 370 (69)

Mean (SD) mg/d

FFQ 728 (361)

24 HR $861(415)$

Mean (SD)

$24 \mathrm{HR} 0.8(0.3)$

FFQ (children) $1.2(0.6)$

FFQ (parents) $1.0(0.4)$

Sixth and seventh grades

Crude 0.32

Deattenuated 0.47

Young adults

Mean (SD)

FFQ 1073 (356)

WDR 726 (228)

9-12 years Mean

$F$ FQ 1576

24 HR 1003

Mean (sD) (mg)

24 HR 1093 (454)

YAQ 1159 (417)

Mean and (sD) (mg)

WDR 813 (218)

FFQ 1016 (273)

11-12 years

Mean (DS)

FFQ 855

24 HR 1008
Children

Mean (SD)

FFQ 1039 (372)

14-16 years

Mean

FFQ 1873

24 HR 1159

13-14 years

Mean (SD)

FFQ 856

$24 \mathrm{HR} 98$ 
NS British Journal of Nutrition

Table 4. Continued

\begin{tabular}{|c|c|c|c|c|}
\hline $\begin{array}{l}\text { Author/year } \\
\text { publication/country/ } \\
\text { (quality index) }\end{array}$ & n/age group & $\begin{array}{l}\text { Methods/ } \\
\text { no FFQ items, } \\
\text { portion sizes }\end{array}$ & Correlation between methods/other statistics & \\
\hline $\begin{array}{l}\text { Mølgaard et al. } \\
(1998)^{(27)} \text { Denmark } \\
(2) \\
\text { Slater et al. } \\
(2003)^{(20)} \text { Brazil (4) }\end{array}$ & $\begin{array}{l}n 23 \\
13-14 \text { years } \\
\text { Adolescents } \\
n 79 \\
14-18 \text { years } \\
\text { Adolescents }\end{array}$ & $\begin{array}{l}\text { FFQ } v .3 \mathrm{~d} \text { WDR } \\
\text { eighty-eight items } \\
\text { HH measures } \\
\text { FFQ v. three } 24 \mathrm{HR} \\
\text { seventy-six items. } \\
\text { Reference portion size } \\
\text { listed. }\end{array}$ & $\begin{array}{l}\text { CC } 0.62 \\
\text { Non-adjusted } 0.61^{\star \star} \\
\text { Energy adjusted } 0.51^{\star \star} \\
\text { Deattenuated } 0.70\end{array}$ & $\begin{array}{l}\text { Median }\left(P_{25}-P_{75}\right)(\mathrm{mg}) \\
\text { FFQ } 1102(931-1339) \\
\text { WDR } 924(727-1145) \\
\text { Mean }(\mathrm{sD})(\mathrm{mg}) \\
\text { 24 HR } 584(384) \\
\text { FFQ } 561(223)\end{array}$ \\
\hline $\begin{array}{l}\text { Andersen et al. } \\
(1995)^{(33)} \text { Norway } \\
(3)\end{array}$ & $\begin{array}{l}n 49 \\
\text { Adolescents }\end{array}$ & $\begin{array}{l}\text { FFQ } v .7 \mathrm{~d} \text { WDR } \\
190 \text { items } \\
\mathrm{HH} \text { measures }\end{array}$ & $\begin{array}{l}\text { Unadjusted } 0.54 \\
\text { Energy adjusted } 0.54\end{array}$ & $\begin{array}{l}\text { Median FFQ: } 1178(\mathrm{mg}) \\
\text { Median WDR: } 936(\mathrm{mg})\end{array}$ \\
\hline \multicolumn{5}{|l|}{$\mathrm{Mg}$} \\
\hline $\begin{array}{l}\text { Marriott et al. } \\
(2008)^{(8)} \text { UK (3.5) }\end{array}$ & $\begin{array}{l}n 50 \\
6 \text { months } \\
\text { Infants }\end{array}$ & $\begin{array}{l}\text { FFQ } v .4 \text { d WDR } \\
\text { thirty-four items } \\
\text { HH measures }\end{array}$ & $\begin{array}{l}\text { Energy adjusted } \\
\text { CC } 0.68\end{array}$ & $\begin{array}{l}\text { Median }(\mathrm{mg}) \\
\text { FFQ } 86.7 \\
\text { WDR } 78.7\end{array}$ \\
\hline $\begin{array}{l}\text { Marriott et al. } \\
(2008)^{(9)} \text { UK (3.5) }\end{array}$ & $\begin{array}{l}n 50 \\
12 \text { months } \\
\text { Infants }\end{array}$ & $\begin{array}{l}\text { FFQ v. } 4 \text { d WDR } \\
\text { seventy-eight items } \\
\text { HH measures }\end{array}$ & $\begin{array}{l}\text { Energy adjusted } \\
\text { CC } 0.57\end{array}$ & $\begin{array}{l}\text { Median }(\mu \mathrm{g}) \\
\text { FFQ } 142 \\
\text { WDR } 129\end{array}$ \\
\hline $\begin{array}{l}\text { Blum et al. }(1999)^{(24)} \\
\text { USA (2.5) }\end{array}$ & $\begin{array}{l}n 233 \\
1-5 \text { years } \\
\text { Infants } \\
\text { Preschool children }\end{array}$ & $\begin{array}{l}\mathrm{FFQ} v \text {. three } 24 \mathrm{HR} \\
\text { eighty-four items. }\end{array}$ & CC 0.63 & $\begin{array}{l}\text { Mean }(\mathrm{SD})(\mathrm{mg}) \\
24 \mathrm{HR} 225(69) \\
\text { FFQ } 220(60)\end{array}$ \\
\hline $\begin{array}{l}\text { Rockett et al. } \\
(1997)^{(29)} \text { USA (3.5) }\end{array}$ & $\begin{array}{l}n 261 \\
9-18 \text { years } \\
\text { Children } \\
\text { Adolescents }\end{array}$ & $\begin{array}{r}\text { YAQ } v \text {. three } 24 \mathrm{HR} \\
131 \text { items. Standard } \\
\text { reference portions }\end{array}$ & $\begin{array}{l}9-13 \text { years CC } 0.51 \\
14-18 \text { years CC } 0.54\end{array}$ & $\begin{array}{l}\text { Mean }(\mathrm{sD})(\mathrm{mg}) \\
24 \mathrm{HR} 278(101) \\
\text { YAQ } 285(88)\end{array}$ \\
\hline $\begin{array}{l}\text { Andersen et al. } \\
(1995)^{(33)} \text { Norway } \\
(3)\end{array}$ & $\begin{array}{l}n 49 \\
\text { Adolescents }\end{array}$ & $\begin{array}{l}\text { FFQ } v .7 \mathrm{~d} \text { WDR } \\
190 \text { items HH measures }\end{array}$ & $\begin{array}{l}\text { Unadjusted } 0.49 \\
\text { Energy adjusted } 0.51\end{array}$ & $\begin{array}{l}\text { Median FFQ: } 405(\mathrm{mg}) \\
\text { Median WDR: } 292(\mathrm{mg})\end{array}$ \\
\hline \multicolumn{5}{|l|}{$\mathrm{P}$} \\
\hline $\begin{array}{l}\text { Marriott et al. } \\
(2008)^{(8)} \text { UK (3.5) }\end{array}$ & $\begin{array}{l}n 50 \\
6 \text { months } \\
\text { Infants }\end{array}$ & $\begin{array}{l}\text { FFQ v. } 4 \mathrm{~d} \text { WDR } \\
\text { thirty-four items } \\
\mathrm{HH} \text { measures }\end{array}$ & $\begin{array}{l}\text { Energy adjusted } \\
\text { CC } 0.77\end{array}$ & $\begin{array}{l}\text { Median }(\mathrm{mg}) \\
\text { FFQ } 422 \\
\text { WDR } 409\end{array}$ \\
\hline $\begin{array}{l}\text { Marriott et al. } \\
(2008)^{(9)} \text { UK (3.5) }\end{array}$ & $\begin{array}{l}n 50 \\
12 \text { months } \\
\text { Infants }\end{array}$ & $\begin{array}{l}\text { FFQ v. } 4 \mathrm{~d} \text { WDR } \\
\text { seventy-eight items } \\
\text { HH measures }\end{array}$ & $\begin{array}{l}\text { Energy adjusted } \\
\text { CC } 0.58\end{array}$ & $\begin{array}{l}\text { Median }(\mu \mathrm{g}) \\
\text { FFQ } 833 \\
\text { WDR } 768\end{array}$ \\
\hline $\begin{array}{l}\text { Field et al. (1998) } \\
\text { USA (4) }\end{array}$ & $\begin{array}{l}n 109 \\
\text { Students from fourth to }\end{array}$ & $\begin{array}{l}\mathrm{FFQ} v \text {. four } 24 \mathrm{HR} \\
\text { ninety-seven items }\end{array}$ & $\begin{array}{l}\text { Fourth and fifth grades } \\
\text { Crude } 0.25\end{array}$ & $\begin{array}{l}\text { Sixth and seventh grades } \\
\text { Crude } 0.29\end{array}$ \\
\hline & $\begin{array}{l}\text { seventh grade } \\
\text { Children }\end{array}$ & $\begin{array}{l}\text { Standard reference } \\
\text { portions used. }\end{array}$ & Deattenuated 0.33 & Deattenuated 0.40 \\
\hline $\begin{array}{l}\text { Rockett et al. } \\
(1997)^{(29)} \text { USA (3.5) }\end{array}$ & $\begin{array}{l}\text { n } 261 \\
9-18 \text { years } \\
\text { Children } \\
\text { Adolescents }\end{array}$ & $\begin{array}{l}\text { YAQ } v \text {. three } 24 \mathrm{HR} \\
131 \text { items. Standard } \\
\text { reference portions }\end{array}$ & $\begin{array}{l}9-13 \text { years } \mathrm{CC} 0.47 \\
14-18 \text { years } \mathrm{CC} 0.61\end{array}$ & $\begin{array}{l}\text { Mean }(\mathrm{sD})(\mathrm{mg}) \\
24 \mathrm{HR} 1381(473) \\
\text { YAQ } 1515(446)\end{array}$ \\
\hline $\begin{array}{l}\text { Mølgaard et al. } \\
(1998)^{(27)} \text { Denmark } \\
(2)\end{array}$ & $\begin{array}{l}n 23 \\
13-14 \text { years } \\
\text { Adolescents }\end{array}$ & $\begin{array}{l}\text { FFQ } v .3 \mathrm{~d} \text { WDR } \\
\text { eighty-eight items } \\
\text { HH measures }\end{array}$ & CC 0.56 & $\begin{array}{l}\text { Median }\left(\mathrm{P}_{25-} \mathrm{P}_{75}\right)(\mathrm{mg}) \\
\text { FFQ } 1191(1027-1538) \\
\text { WDR } 1081(799-1360)\end{array}$ \\
\hline \multicolumn{5}{|l|}{$\mathrm{Fe}$} \\
\hline $\begin{array}{l}\text { Marriott et al. } \\
(2008)^{(8)} \text { UK (3.5) }\end{array}$ & $\begin{array}{l}n 50 \\
6 \text { months } \\
\text { Infants }\end{array}$ & $\begin{array}{l}\text { FFQ } v .4 \mathrm{~d} \text { WDR } \\
\text { thirty-four items } \\
\text { HH measures }\end{array}$ & $\begin{array}{l}\text { Energy adjusted } \\
\text { CC } 0.78\end{array}$ & $\begin{array}{l}\text { Median }(\mathrm{mg}) \\
\text { FFQ } 7.0 \\
\text { WDR } 6.9\end{array}$ \\
\hline
\end{tabular}


NS British Journal of Nutrition

Author/year

publication/country/

(quality index)

\begin{tabular}{ll}
\hline Marriott et al. & $n 50$ \\
$(2008)^{(9)}$ UK (3.5) & 12 months \\
& Infants \\
Williams \& Innis & $n 148$ \\
$(2005)^{(17)}$ Canada & $8-26$ months \\
$(5)$ & Infants
\end{tabular}

Andersen et al. $\quad n 64$

$\begin{array}{ll}\text { (4.5) } & \text { Infants } \\ \text { Blum et al. (1999) (24) } & n 233\end{array}$

USA (2.5)

Andersen et al.

$(2004)^{(18,19)}$ Norway

(5)

Räsänen L.

$(1979)^{(39)}$ Finland

(5)

Infants
$n 233$

$1-5$ years

Infants

chool children

187

2 years old

Preschool children

n 741

5-13 years

Preschool children

Children

Adolescents

Holmes et al.

$(2008)^{(10)}$ UK (3)

$n 124$

2-17 years

Preschool children

Children

Adolescents

Bellù et al. (1996) ${ }^{(30)}$

Italy (3)

Lillegaard et al.

$(2007)^{(12)}$ Norwa

(3.5)

$n 333$

$8-10$ years

Children

9 years old

Children

Field et al. (1998)

USA (4)

Bellù et al. (1995)

Italy (3.5) n 109

Students from fourth to

Children
$n 88$

$9-12$ years
FC v. 4d WDR

SW v. 4 d WDR

FFQ v. $24 \mathrm{HR}$

116 items.

Methods/

no FFQ items,

FFQ v. 4 d WDR

seventy-eight items

FFQ v. 3d EDR

191 items

FFQ v. BM

3d EDR $v . \mathrm{BM}$

FFQ v. 7d WDR

140 items. HH measures or

food photographs

FFQ $v$. three $24 \mathrm{HR}$

eighty-four items.

FFQ v. $7 d$ WDR

food photogra

CC 0.46

methods/other statistics

Energy adjusted

CC 0.50

CC 0.64

Serum ferritin and total

Fe from $F F Q r=0.33$

Serum ferritin and total

Fe from $3 d$ EDR $r=0.19$

Absolute intake

0.62

CC 0.51

$24 \mathrm{HR}$ v. 4 d WDR

Males-Mean difference

2-10 years $0.1(P=0.864)$

$11-17$ years $1.4(P=0.055)$

Males-Mean difference

$2-10$ years $0.5(P=0.356)$

$11-17$ years $0.1(P=0.847)$

Males-Mean difference

$2-10$ years $-1.1(P=0.101)$

$11-17$ years $-1.2(P=0.237)$

\section{Girls Mean (SD)}

24 HR 5.6 (2.6)

FFQ $5.3(0.9)$

PFD v. 4d WDR

277 items $\mathrm{HH}$ measures or

colour photographs

WDR $8.4(7.1-9.5)$

CC $0.32^{*}$

FFQ $v$. four $24 \mathrm{HR}$

ninety-seven items. Stan-

dard reference portions

used.

FFQ v. $7 d$ EDR

Fouth and fith grades

Crude 0.20

Deattenuated 0.28

Raw nutrient 0.31

Nutrient density 0.18

116 items. Reference por-
Boys-Median $\left(P_{25}-P_{75}\right)(\mathrm{mg})$

Median $(\mu \mathrm{g})$

FFQ 8.48

WDR 5.20

Median (mg)

FFQ 9.6; EDR 7.1 tion sizes
Median $\left(\mathrm{P}_{25}-\mathrm{P}_{75}\right)(\mathrm{mg})$

FFQ $8.9(6.7-12.9)$

WDR $5.6(3.6-10 \cdot 2)^{\star \star \star}$

Mean (SD) (mg)

24 HR 16 (4)

FFQ 12 (6)

Median $\left(\mathrm{P}_{25}-\mathrm{P}_{75}\right)(\mathrm{mg})$

FFQ 46.5 (5.2-8.0)

Mean DH: 17.6; 24 HR: $11 \cdot 6$

Females-Mean difference

$2-10$ years $0.7(P=0.157)$

$11-17$ years $1.1(P=0.072)$

Females-Mean difference

$2-10$ years $1.4(P=0.000)$

$11-17$ years $0.4(P=0.348)$

Females-Mean difference

$2-10$ years -0.5

$(P=0.358)$

$11-17$ years $0.7(P=0.398)$

Boys Mean (SD)

(2)

Q $5.0(0.9)$

Girls-Median $\left(\mathrm{P}_{25}-\mathrm{P}_{75}\right)(\mathrm{mg})$

PFD 8.4 (7.1-10.8)

WDR $7.1(6.4-8.0)$

CC $0.52^{* \star \star}$

Sixth and seventh grades

Crude 0.29

Deattenuated 0.40

Mean (SD)

EDR $5.1(0.67)$ 


\begin{tabular}{|c|c|c|c|c|}
\hline $\begin{array}{l}\text { Author/year } \\
\text { publication/country/ } \\
\text { (quality index) }\end{array}$ & n/age group & $\begin{array}{l}\text { Methods/ } \\
\text { no FFQ items, } \\
\text { portion sizes }\end{array}$ & Correlation between methods/other statistics & \\
\hline $\begin{array}{l}\text { Rockett et al. } \\
(1997)^{(29)} \text { USA (3.5) }\end{array}$ & $\begin{array}{l}n 261 \\
9-18 \text { years } \\
\text { Children } \\
\text { Adolescents }\end{array}$ & $\begin{array}{l}\text { YAQ v. three } 24 \mathrm{HR} \\
131 \text { items. Standard refer- } \\
\text { ence portions }\end{array}$ & $\begin{array}{l}9-13 \text { years CC } 0.47 \\
14-18 \text { years CC } 0.59\end{array}$ & $\begin{array}{l}\text { Mean }(\mathrm{SD})(\mathrm{mg}) \\
24 \mathrm{HR} 18(9) \\
\text { YAQ } 18(8)\end{array}$ \\
\hline $\begin{array}{l}\text { Slater et al. } \\
(2003)^{(20)} \text { Brazil (4) }\end{array}$ & $\begin{array}{l}n 79 \\
14-18 \text { years } \\
\text { Adolescents }\end{array}$ & $\begin{array}{l}\mathrm{FFQ} v \text {. three } 24 \mathrm{HR} \\
\text { seventy-six items. } \\
\text { Reference portion size } \\
\text { listed. }\end{array}$ & $\begin{array}{l}\text { Non-adjusted } 0.46^{\star \star} \\
\text { Energy adjusted } 0.17 \\
\text { Deattenuated } 0.22\end{array}$ & $\begin{array}{l}\text { Mean }(\mathrm{sD})(\mathrm{mg}) \\
24 \mathrm{HR} 11.7(5 \cdot 6) \\
\text { FFQ } 8.4(2.4)\end{array}$ \\
\hline $\begin{array}{l}\text { Andersen et al. } \\
(1995)^{(33)} \text { Norway } \\
\text { (3) }\end{array}$ & $\begin{array}{l}n 49 \\
\text { Adolescents }\end{array}$ & $\begin{array}{l}\text { FFQ } v .7 d \text { WDR } \\
190 \text { items HH measures }\end{array}$ & $\begin{array}{l}\text { Unadjusted } 0.52 \\
\text { Energy adjusted } 0.39\end{array}$ & $\begin{array}{l}\text { Median FFQ: } 12(\mathrm{mg}) \\
\text { Median WDR: } 9(\mathrm{mg})\end{array}$ \\
\hline \multicolumn{5}{|l|}{$\mathrm{Zn}$} \\
\hline $\begin{array}{l}\text { Marriott et al. } \\
(2008)^{(8)} \text { UK (3.5) }\end{array}$ & $\begin{array}{l}n \text { equals; } 50 \\
6 \text { months } \\
\text { Infants }\end{array}$ & $\begin{array}{l}\text { FFQ } v .4 \mathrm{~d} \text { WDR } \\
\text { thirty-four items } \mathrm{HH} \\
\text { measures }\end{array}$ & $\begin{array}{l}\text { Energy adjusted } \\
\text { CC } 0.83\end{array}$ & $\begin{array}{l}\text { Median (mg) } \\
\text { FFQ } 5 \cdot 2 \\
\text { WDR } 4.7\end{array}$ \\
\hline $\begin{array}{l}\text { Marriott et al. } \\
(2008)^{(9)} \text { UK (3.5) }\end{array}$ & $\begin{array}{l}n 50 \\
12 \text { months } \\
\text { Infants }\end{array}$ & $\begin{array}{l}\text { FFQ } v .4 \mathrm{~d} \text { WDR } \\
\text { seventy-eight items } \mathrm{HH} \\
\text { measures }\end{array}$ & $\begin{array}{l}\text { Energy adjusted } \\
\text { CC } 0.48\end{array}$ & $\begin{array}{l}\text { Median }(\mu \mathrm{g}) \\
\text { FFQ } 6.25 \\
\text { WDR } 4.54\end{array}$ \\
\hline $\begin{array}{l}\text { Blum et al. }(1999)^{(24)} \\
\text { USA (2.5) }\end{array}$ & $\begin{array}{l}n 233 \\
1-5 \text { years } \\
\text { Infants } \\
\text { Preschool children }\end{array}$ & $\begin{array}{l}\text { FFQ } v \text {. three } 24 \mathrm{HR} \\
\text { eighty-four items. }\end{array}$ & CC 0.31 & $\begin{array}{l}\text { Mean }(\mathrm{sD})(\mathrm{mg}) \\
24 \mathrm{HR} 10(7) \\
\text { FFQ } 11(5)\end{array}$ \\
\hline $\begin{array}{l}\text { Rockett et al. } \\
(1997)^{(29)} \text { USA (3.5) }\end{array}$ & $\begin{array}{l}n 261 \\
9-18 \text { years } \\
\text { Children } \\
\text { Adolescents }\end{array}$ & $\begin{array}{r}\text { YAQ } v \text {. three } 24 \mathrm{HR} \\
131 \text { items. Standard } \\
\text { reference portions }\end{array}$ & $\begin{array}{l}9-13 \text { years CC } 0.36 \\
14-18 \text { years CC } 0.59\end{array}$ & $\begin{array}{l}\text { Mean }(\mathrm{SD})(\mathrm{mg}) \\
24 \mathrm{HR} 12(6) \\
\text { YAQ } 14(7)\end{array}$ \\
\hline \multicolumn{5}{|c|}{ Adurescents } \\
\hline $\begin{array}{l}\text { Marriott et al. } \\
(2008)^{(8)} \text { UK (3.5) }\end{array}$ & $\begin{array}{l}n 50 \\
6 \text { months } \\
\text { Infants }\end{array}$ & $\begin{array}{l}\text { FFQ } v .4 \mathrm{~d} \text { WDR } \\
\text { thirty-four items } \mathrm{HH} \\
\text { measures }\end{array}$ & $\begin{array}{l}\text { Energy adjusted } \\
\text { CC } 0.55\end{array}$ & $\begin{array}{l}\text { Median (mg) } \\
\text { FFQ } 0.46 \\
\text { WDR } 0.41\end{array}$ \\
\hline $\begin{array}{l}\text { Marriott et al. } \\
(2008)^{(9)} \text { UK (3.5) }\end{array}$ & $\begin{array}{l}n 50 \\
12 \text { months } \\
\text { Infants }\end{array}$ & $\begin{array}{l}\text { FFQ } v .4 \mathrm{~d} \text { WDR } \\
\text { seventy-eight items } \mathrm{HH} \\
\text { measures }\end{array}$ & $\begin{array}{l}\text { Energy adjusted } \\
\text { CC } 0.63\end{array}$ & $\begin{array}{l}\text { Median }(\mu \mathrm{g}) \\
\text { FFQ } 0.64 \\
\text { WDR } 0.43\end{array}$ \\
\hline $\begin{array}{l}\text { Rockett et al. } \\
(1997)^{(29)} \text { USA (3.5) }\end{array}$ & $\begin{array}{l}n 261 \\
9-18 \text { years } \\
\text { Children } \\
\text { Adolescents }\end{array}$ & $\begin{array}{l}\text { YAQ v. three } 24 \mathrm{HR} \\
131 \text { items. Standard } \\
\text { reference portions }\end{array}$ & $\begin{array}{l}9-13 \text { years CC } 0.47 \\
14-18 \text { years CC } 0.61\end{array}$ & $\begin{array}{l}\text { Mean }(\mathrm{SD})(\mathrm{mg}) \\
24 \mathrm{HR} 1.4(0.8) \\
\text { YAQ } 1.7(0.8)\end{array}$ \\
\hline
\end{tabular}

WDR, weighed dietary record; CC, correlation coefficient; $24 \mathrm{HR}, 24 \mathrm{~h}$ recall; EDR, estimated dietary record; YAQ, Youth/Adolescent Questionnaire; BM, biomarker; HH measures, household measures; FC, food checklist; SW, semi-weighed method; DH, diet history; PFD, pre-coded food diary; IIC, intra-class correlation coefficients.

Mean values were significantly different: ${ }^{*} P<0.05 ;{ }^{* *} P<0.01 ; * \star * * 0.001$. 
(a)
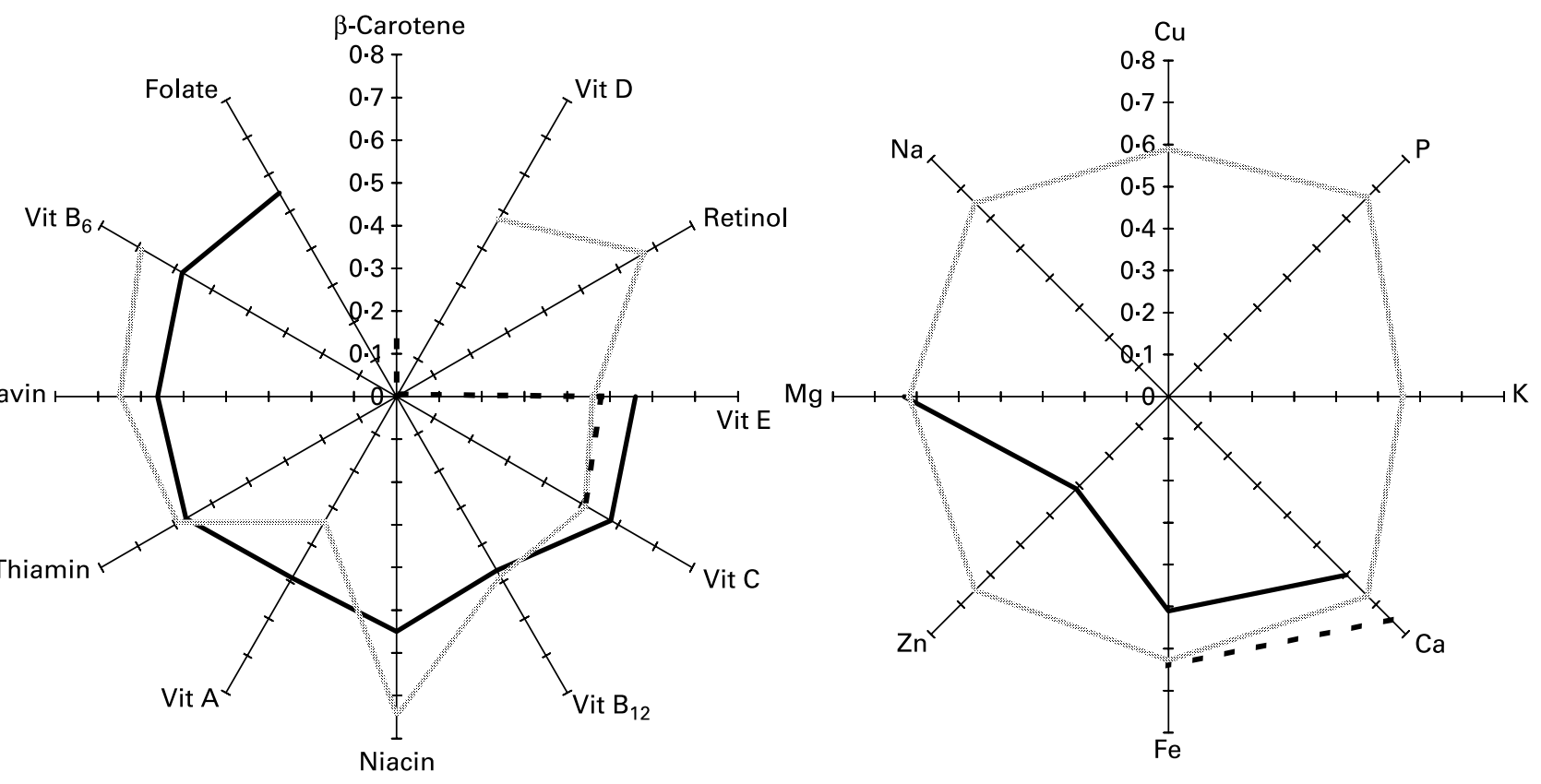

(b)
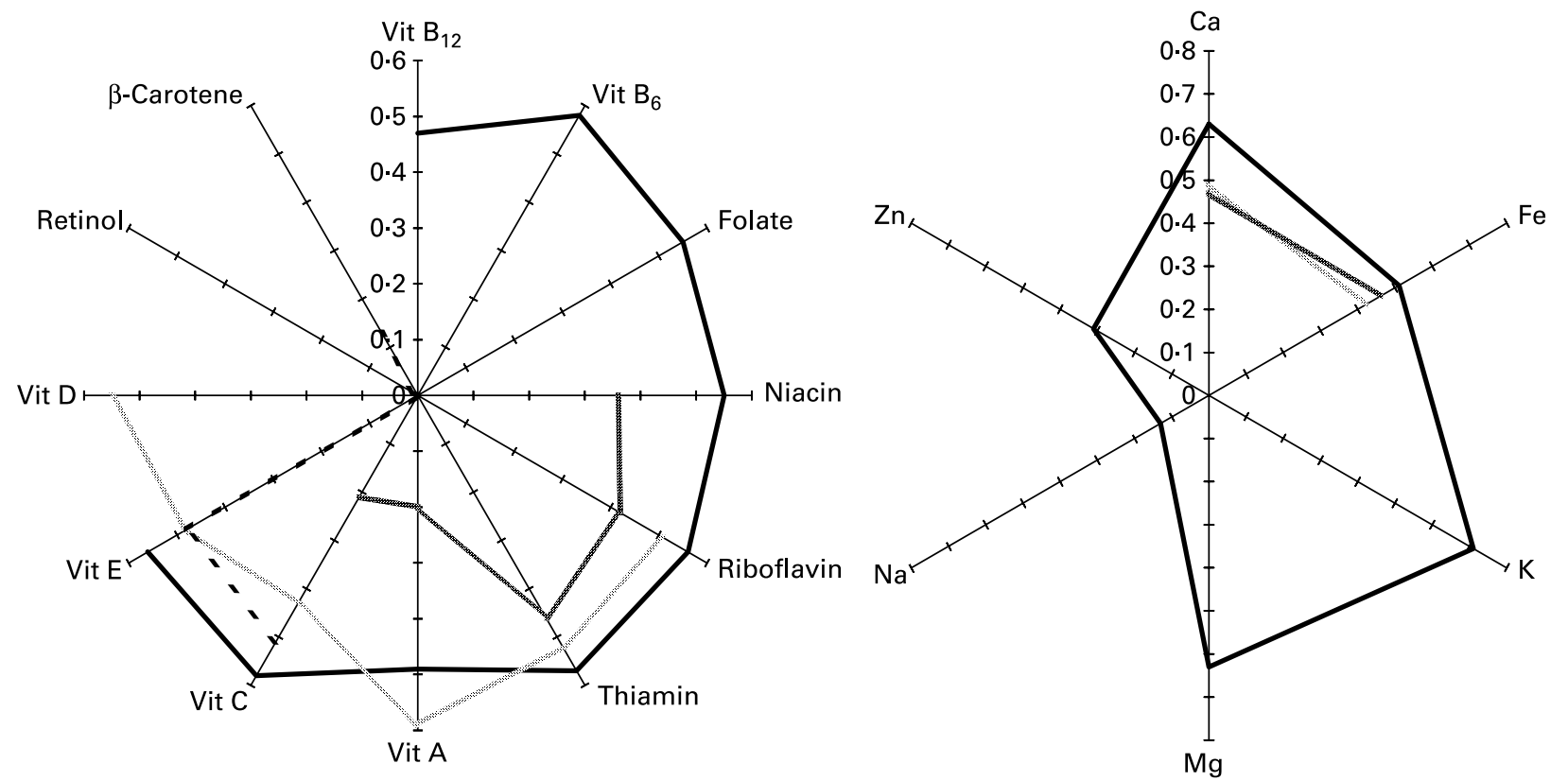

Fig. 2. Comparison of different dietary assessment methods in infants (1-23 months) and preschool children (2-5 years) by vitamins and minerals (mean of quality weighted correlation coefficients) $24 \mathrm{HR}, 24 \mathrm{~h}$ recall; EDR, estimated dietary record; WDR, weighed dietary record; BM, biomarker; DH, diet history. (a) Infants (three or more studies: vitamin D, four studies FFQ $v$. WDR; vitamin E, three studies FFQ $v$. WDR; vitamin C, three studies FFQ $v$. WDR; thiamin, three studies FFQ $v$. WDR; riboflavin, four studies FFQ $v$. WDR; calcium, four studies FFQ $v$. WDR; iron, four studies FFQ $v$. WDR). (one study ${ }^{(24)}$ ); : :

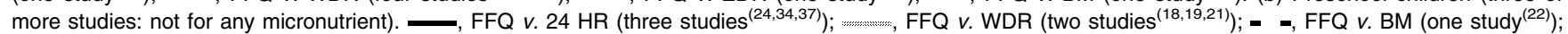
m. $24 \mathrm{HR}$ v. DH (one study $\left.{ }^{(39)}\right)$.

the number of food items were observed (7-191 items). In addition, five studies were classified into group 1 with a reference method that reflected short-term intake, in which one applied $24 \mathrm{~h}$ recalls ${ }^{(24)}$, three used $\operatorname{WDR}^{(8,9,21)}$ and one applied EDR ${ }^{(17)}$. Likewise, another study was classified into group 2, where the reference method reflected long-term intake; this study used WDR as the reference method ${ }^{(2)}$. Finally, two studies utilised BM as the reference $\operatorname{method}^{(17,22)}$, of which one ${ }^{(17)}$ presented validation of more than one instrument. The number of repeated $24 \mathrm{~h}$ recalls ranged from 3 to $4 \mathrm{~d}$ of administration. Dietary records varying in the number of recording days (from 3 to $7 \mathrm{~d}$ ) were used as the reference method in a total of five studies.

Comparison of different dietary assessment methods in infants by vitamins and minerals is presented in Fig. 2. This figure shows that WDR used as the reference method for evaluating FFQ presented better correlations for several micronutrients than the other methods in this population 
Table 5. Classification of the dietary assessment methods for infants, children and adolescents according to the weighted mean of the correlations of each micronutrient (including three or more studies)

\begin{tabular}{|c|c|c|c|c|}
\hline \multirow[b]{2}{*}{ Micronutrient/age group } & \multicolumn{4}{|c|}{ Correlation* } \\
\hline & FFQ $v$. WDR & FFQ v. (WDR + EDR) & FFQ v. $24 \mathrm{HR}$ & $(F F Q+Y A Q) v .24 \mathrm{HR}$ \\
\hline Vitamin D infants & $\mathrm{A}=0.48$ (four studies) & & & \\
\hline Vitamin C infants & $\mathrm{G}=0.51$ (three studies) & & & \\
\hline Vitamin $E$ infants & $A=0.46$ (three studies) & & & \\
\hline Thiamin infants & $\mathrm{G}=0.59$ (three studies) & & & \\
\hline Riboflavin infants & $\mathrm{G}=0.65$ (three studies) & & & \\
\hline \multicolumn{5}{|l|}{$\mathrm{Ca}$} \\
\hline Infants & $\mathrm{G}=0.67$ (four studies) & $\mathrm{G}=0.69$ (five studies) & & \\
\hline Preschool children & & $A=0.50$ (four studies) & & \\
\hline Children & & $A=0.50$ (four studies) & $A=0.48$ (five studies) & $\mathrm{A}=0.49$ (six studies) \\
\hline Adolescents & $\mathrm{G}=0.58$ (three studies) & & $\mathrm{A}=0.50$ (three studies) & $\mathrm{G}=0.52$ (four studies) \\
\hline $\begin{array}{l}\mathrm{Fe} \\
\text { Infants }\end{array}$ & $\mathrm{G}=0.63$ (three studies) & $\mathrm{G}=0.63$ (four studies) & & \\
\hline
\end{tabular}

WDR, weighed dietary record; EDR, estimated dietary record; $24 \mathrm{HR}, 24 \mathrm{~h}$ recall; YAQ, Youth/Adolescent Questionnaire.

${ }^{*}$ Correlation V: very good (>0.7); G: good (0.51-0.70); A: acceptable (0.30-0.50); P: poor $(<0.30)$

group. However, we must emphasise that this result is probably due to the fact that $57 \%$ of the studies analysed in this group used WDR as the reference method.

Table 5 presents the classification of the dietary methods utilised for studies in infants according to the mean of the correlation coefficients for each micronutrient weighted by the quality of different validation studies included in this review. Methods analysed met the criteria of having at least three studies, thus providing sufficient data to conduct quantitative estimates for each micronutrient ${ }^{(40)}$. Vitamin D and vitamin $\mathrm{E}$ intake analysed using FFQ $v$. WDR showed acceptable correlations. Comparing these methods, we observed that vitamin $\mathrm{C}$, thiamin, riboflavin, $\mathrm{Ca}$ and $\mathrm{Fe}$ presented a good correlation. Additionally, when FFQ were validated considering WDR and EDR as the reference methods, only $\mathrm{Ca}$ 's correlation increased slightly. The correlation for $\mathrm{Fe}$ was not modified, and for the rest of the micronutrients, there was insufficient data to conduct an analysis. (Table 5)

\section{Preschool children}

For this review, the preschool children group included children aged 2-5 years. Of the thirty-two articles included in the present review, ten showed data on the validation of methods used to assess micronutrient intake in preschool children $^{(10,15,18,21,22,24,26,34,37,39)}$. Eight different FFQ had been validated ${ }^{(15,18,21,22,24,26,34,37)}$, and a $24 \mathrm{~h}$ recall had been validated in two studies ${ }^{(10,39)}$. Some articles presented validation of more than one instrument, of which one study also validated $24 \mathrm{~h}$ recalls ${ }^{(22)}$, and another study validated a food checklist and a semi-weighed method ${ }^{(10)}$. After evaluating the quality of these validation studies, the quality scores obtained ranged from 2.5 to 5 . Different FFQ were validated for which wide variations in the number of food items were observed (7-125 items). Eight studies were classified into group 1 with a reference method that reflected short-term intake, in which two used $\mathrm{WDR}^{(10,21)}$, another two applied $\operatorname{EDR}^{(15,26)}$, three used $24 \mathrm{~h}$ recalls ${ }^{(24,34,38)}$ and one applied a $\mathrm{DH}^{(39)}$. Likewise, one study was classified into group 2, where the reference method reflected long-term intake, in which WDR were applied ${ }^{(18)}$. Finally, one study utilised BM as the reference method, which presented validation of more than one instrument ${ }^{(22)}$. The number of repeated $24 \mathrm{~h}$ recalls ranged from 3 to $4 \mathrm{~d}$ of administration. Dietary records varying in the number of recording days (from 3 to $7 \mathrm{~d}$ ) were used as the reference method in a total of four studies.

Comparison of different dietary assessment methods for vitamin and mineral intake in preschool children is presented in Fig. 2. This figure shows that there were not enough studies to conduct a comparison per micronutrient, as the minimum criterion of three studies per intake assessment method was not met. Moreover, when FFQ were validated applying WDR and EDR as the reference methods, only the correlation for $\mathrm{Ca}$ presented acceptable values ( $r$ 0.50; Table 5 and Fig. 3 ).

Fig. 4 only shows FFQ validation studies that assessed micronutrient intake in infants and preschool children, using a short-term ${ }^{(8,9,15,17,21,24,26,34,37)}$ or a long-term ${ }^{(2,18)}$ dietary assessment instrument or BM as a reference method ${ }^{(17,22)}$. In regards to the reference method that reflected short-term intake, good correlations were observed for niacin, thiamin, vitamins $\mathrm{B}_{6}, \mathrm{D}, \mathrm{C}, \mathrm{E}$, riboflavin, $\mathrm{Ca}, \mathrm{K}, \mathrm{Mg}, \mathrm{Fe}$, and $\mathrm{Zn}$. However, when the reference method used reflected long-term intake, good correlations were observed only for riboflavin and Fe. Additionally, when BM were used as the reference method, a good correlation was observed only for vitamin C. None of the micronutrients analysed showed correlations higher than 0.7 using a short-term or a long-term dietary assessment instrument or BM as the reference method. However, results presented in FFQ validation studies using short-term or long-term dietary instruments or BM as the reference methods based on correlations from only one or two studies should be viewed with caution (Fig. 4). To conduct micronutrient comparisons, there should be at least three or more studies to ensure the robustness of the results obtained.

\section{Children}

This group included children aged 6-12 years. Of the thirtytwo articles included in the present review, seventeen showed data on validation of methods used to assess 
micronutrient intake in children ${ }^{(10-14,16,23,25,26,29-32,35,36}$, ${ }^{38,39)}$. After evaluating the quality of these studies, the quality scores obtained ranged from 2.5 to 5 . Eleven different FFQ $^{(11,13,14,16,23,25,26,30-32,35,38)}$ had been validated, and $24 \mathrm{~h}$ recalls had been validated in three studies ${ }^{(10,36,39)}$. Different FFQ were validated for which wide variations in the number of food items were observed (10-175 items). Of these, it is worth pointing out the Youth/Adolescent Questionnaire $(\mathrm{YAQ})^{(29)}$ that is a self-administered FFQ specifically designed for children aged 9-18 years. Another validated dietary method analysed included $4 \mathrm{~d}$ pre-coded food diaries $^{(12)}$. The pre-coded food diary is scanable and is developed to simplify the work of the respondents as well as of the researcher. Some articles presented validation of more than one instrument, of which one study also validated a food checklist and a semi-weighed method ${ }^{(10)}$, and another study correlated data from $\mathrm{WDR}^{(23)}$. Eleven studies were classified into group 1 with a reference method that reflected short-term intake, in which six studies used $24 \mathrm{HR}^{(11,13,14,25,29,30)}$, two applied $\mathrm{WDR}^{(10,12)}$, only one study used $\mathrm{EDR}^{(26)}$, one applied a $\mathrm{DH}^{(39)}$ and another study observed intakes ${ }^{(36)}$. Likewise, five other studies were classified into group 3, where the reference method reflected long-term intake, two studies used $\mathrm{WDR}^{(16,23)}$, two applied $\mathrm{EDR}^{(31,32)}$ and only one study utilised $24 \mathrm{~h}$ recalls ${ }^{(38)}$ as the reference method. Finally, one study was validated using (a)

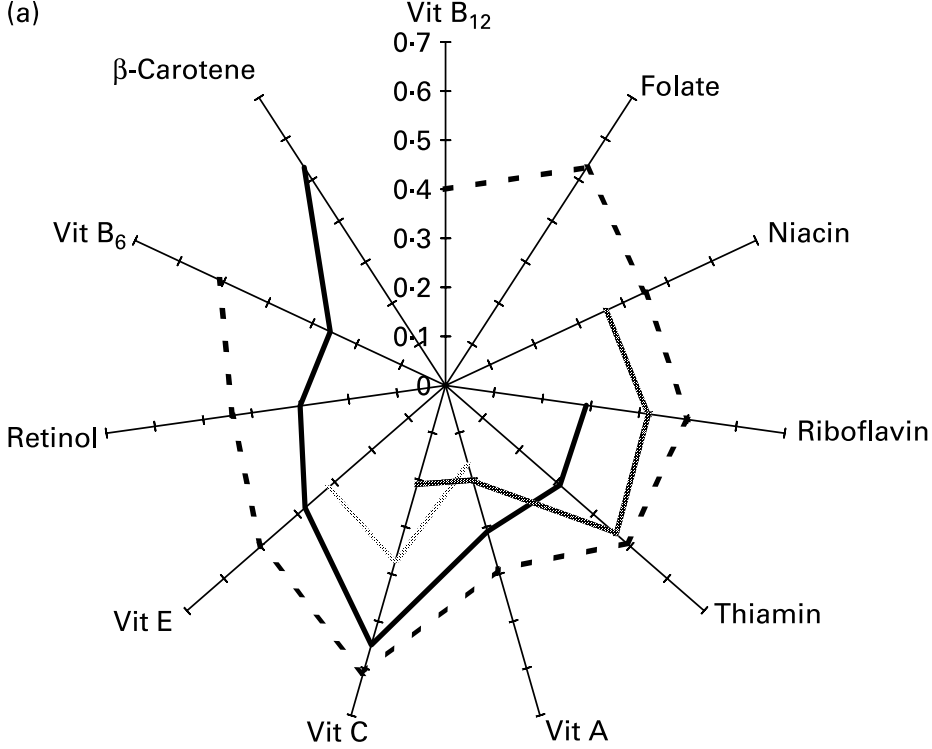

(b)

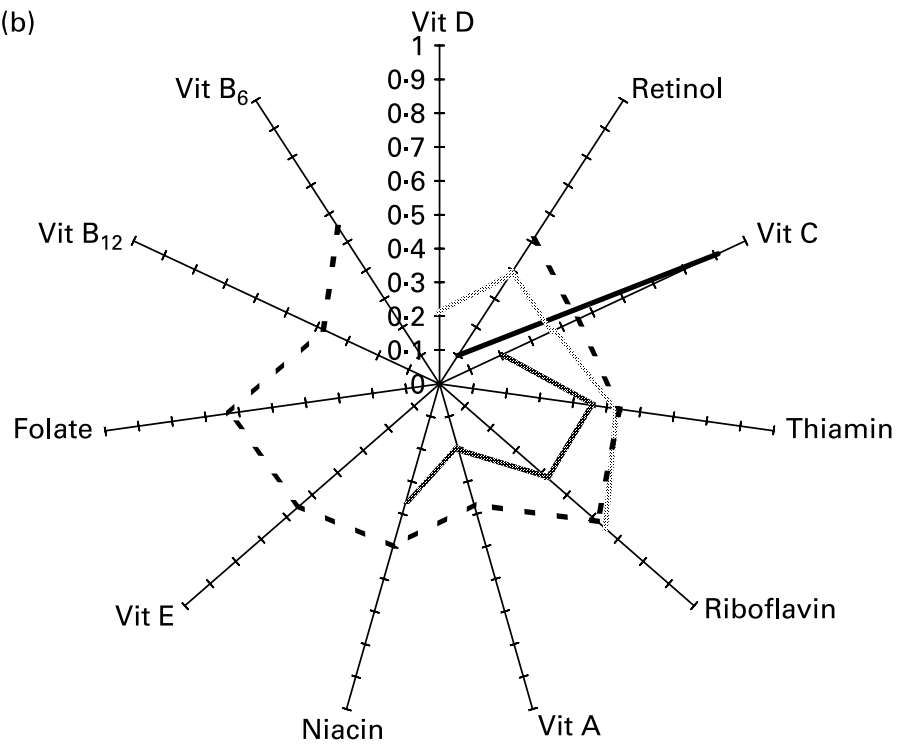

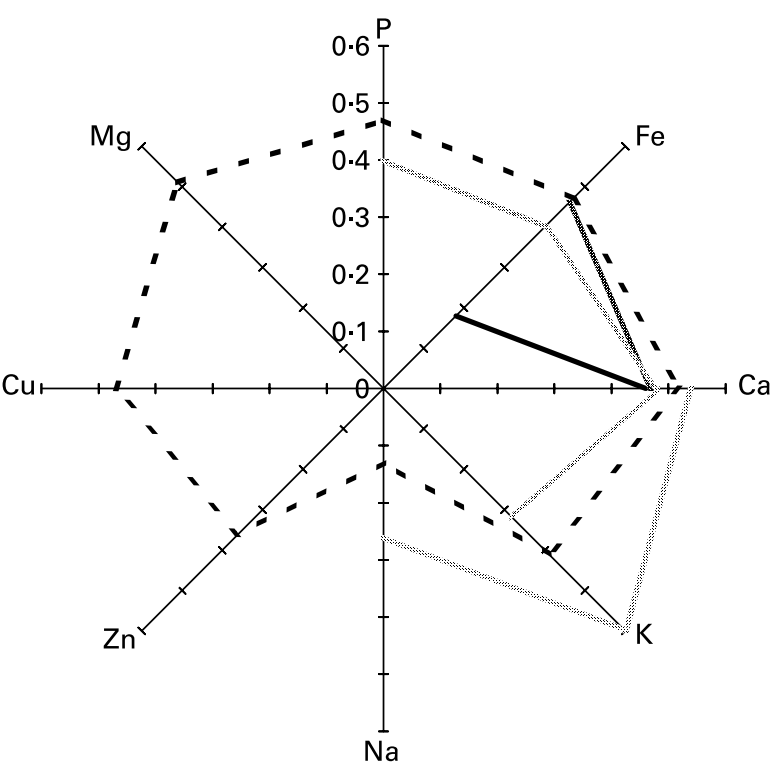

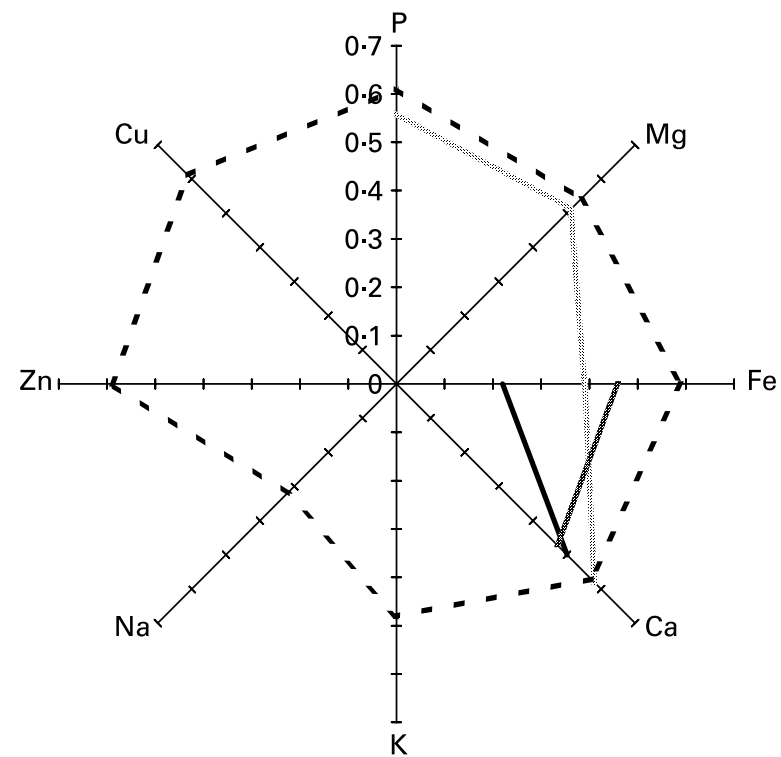

Fig. 3. Comparison of different dietary assessment methods in children (6-12 years) and adolescents (13-10 years) by vitamins and minerals (mean of quality weighted correlation coefficients) $24 \mathrm{HR}, 24 \mathrm{~h}$ recall; EDR, estimated dietary record; WDR, weighed dietary record; DH, dietary history; YAQ, Youth/Adolescent Questionnaire. (a) Children (three or more studies: calcium, five studies FFQ v. $24 \mathrm{HR}$ ). studies $^{(16,23)}$ ); .........., FFQ v. BM (one study ${ }^{(35)}$ ); $\ldots$ (five studies $\left.{ }^{(11,13,16,25,38)}\right)$. (b) Adolescents (three or more studies: calcium: three studies FFQ $v$. $24 \mathrm{HR}$; three studies FFQ $v$. WDR). 2 , FFQ $v$. 24 HR

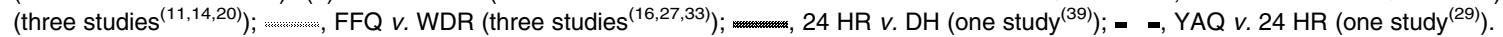




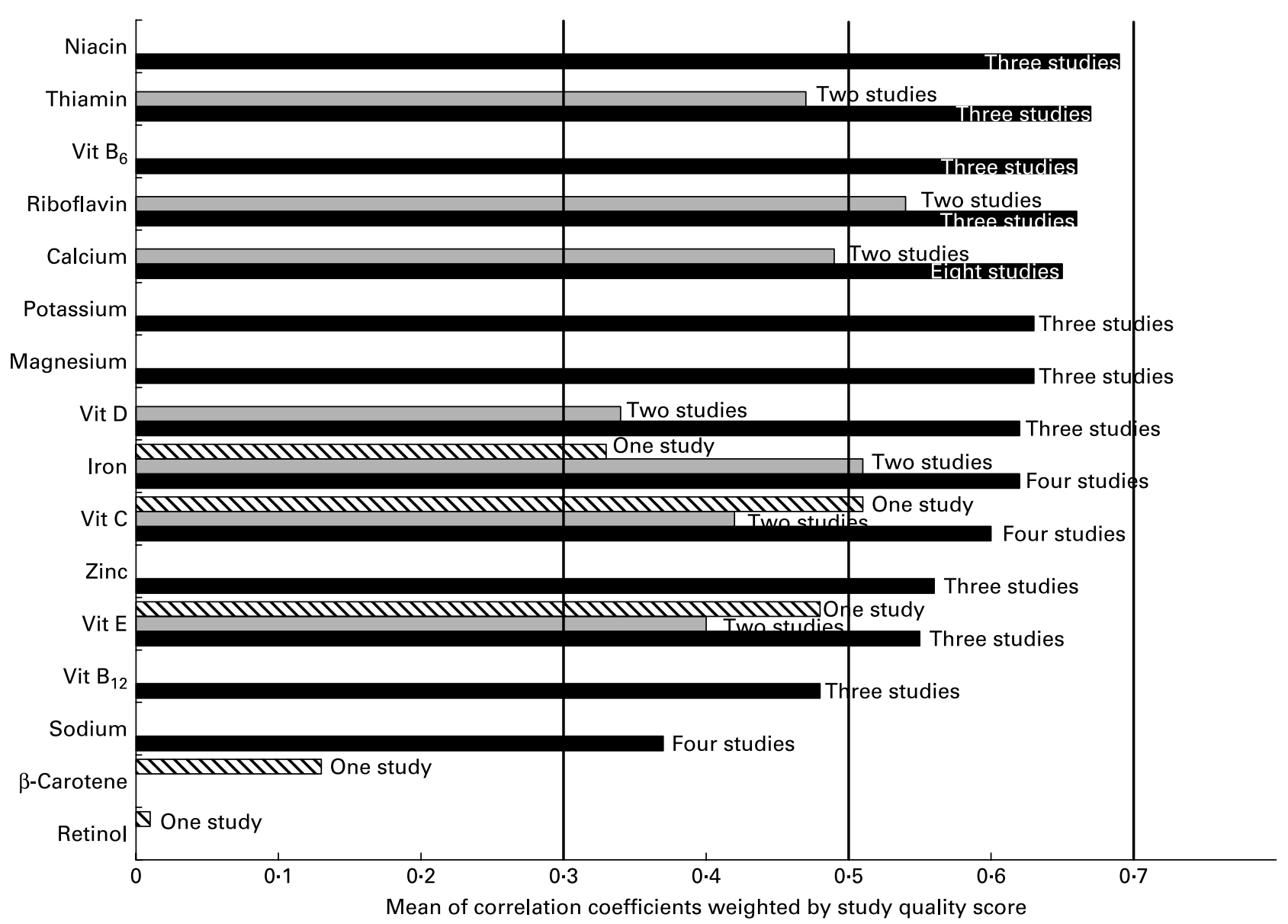

Fig. 4. Validation of FFQ studies that assess micronutrient intake in infants (1-23 months) and preschool children (2-5 years) using as the reference method: short-term or long-term dietary instruments or biomarkers. Correlations: poor $(<0.30)$, acceptable $(0.30-0.50)$, good $(0.51-0.70)$ and very good $(>0.70)$. Three or more studies: sodium, vitamins $B_{12}, E, C, D, B_{6}$, zinc, iron, magnesium, potassium, calcium, riboflavin, thiamin, niacin. $\mathbf{\square}$, Short-term intake ( $\left.<7 \mathrm{~d}\right)$; $\square$, long-term intake ( $\geq 7 \mathrm{~d})$; $\mathbb{\mathbb { Q }}$, biomarkers.

$\mathrm{BM}^{(35)}$. Some articles applied more than one instrument as the reference methods, also employing the use of $\mathrm{BM}^{(23)}$. The number of repeated $24 \mathrm{~h}$ recalls ranged from 1 to 14 administration days. Dietary records varying in the number of recording days (from 1 to $14 \mathrm{~d}$ ) were used as the reference method in a total of seven studies.

Fig. 3 presents the comparison of different dietary assessment methods in children by vitamins and minerals. In this figure, when the $24 \mathrm{HR}$ was used as the reference method, it seemed to obtain better correlations for several micronutrients. However, only five studies were included for measuring $\mathrm{Ca}$ intake, for which FFQ were validated against 24HR, presenting an acceptable correlation.

Additionally, when FFQ were validated utilising WDR and EDR as the reference methods, only the correlation for $\mathrm{Ca}$ presented an acceptable value $(r$ 0.50). Similar correlations were observed when the validity of $\mathrm{Ca}$ intake methods using FFQ (FFQ and YAQ) was assessed by comparing them with $\mathrm{Ca}$ intake estimated by $24 \mathrm{HR}$ ( $r$ 0.48 and $r 0.49$, respectively). The analysis for $\mathrm{Ca}$ was conducted as it met the minimum of having data from at least three studies. However, for the rest of the micronutrients, there were insufficient data to conduct a comparative analysis (Table 5).

\section{Adolescents}

For the present paper, the adolescent age group included children aged 13-18 years. Of the thirty-two articles included in the present review, ten showed data on validation of methods used to assess micronutrient intake in adoles-

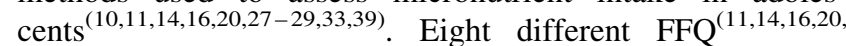
27-29,33,39) had been validated (of which one was the YAQ $)^{(29)}$, and $24 \mathrm{~h}$ recalls had been validated in two studies $^{(10,39)}$. Some articles presented validation of more than one instrument, of which one study also validated a food checklist and a semi-weighed method ${ }^{(10)}$. After evaluating the quality of these studies, the quality scores obtained ranged from $2 \cdot 5$ to 5 . Seven studies were classified into group 1 with a reference method that reflected short-term intake, in which four studies used $24 \mathrm{HR}^{(11,14,20,29)}$, two studies applied $\mathrm{WDR}^{(10,27)}$ and another study administered a $\mathrm{DH}^{(39)}$ In addition, two studies were classified into group 2 with a reference method that reflected long-term intake, in which WDR were used as the reference $\operatorname{method}^{(16,33)}$ and finally, one study used $\mathrm{BM}^{(28)}$. The number of repeated $24 \mathrm{~h}$ recalls ranged from 1 to 3 . Different FFQ were validated for which wide variations in the number of food items were observed (10-190 items). Dietary records varying in the number of 


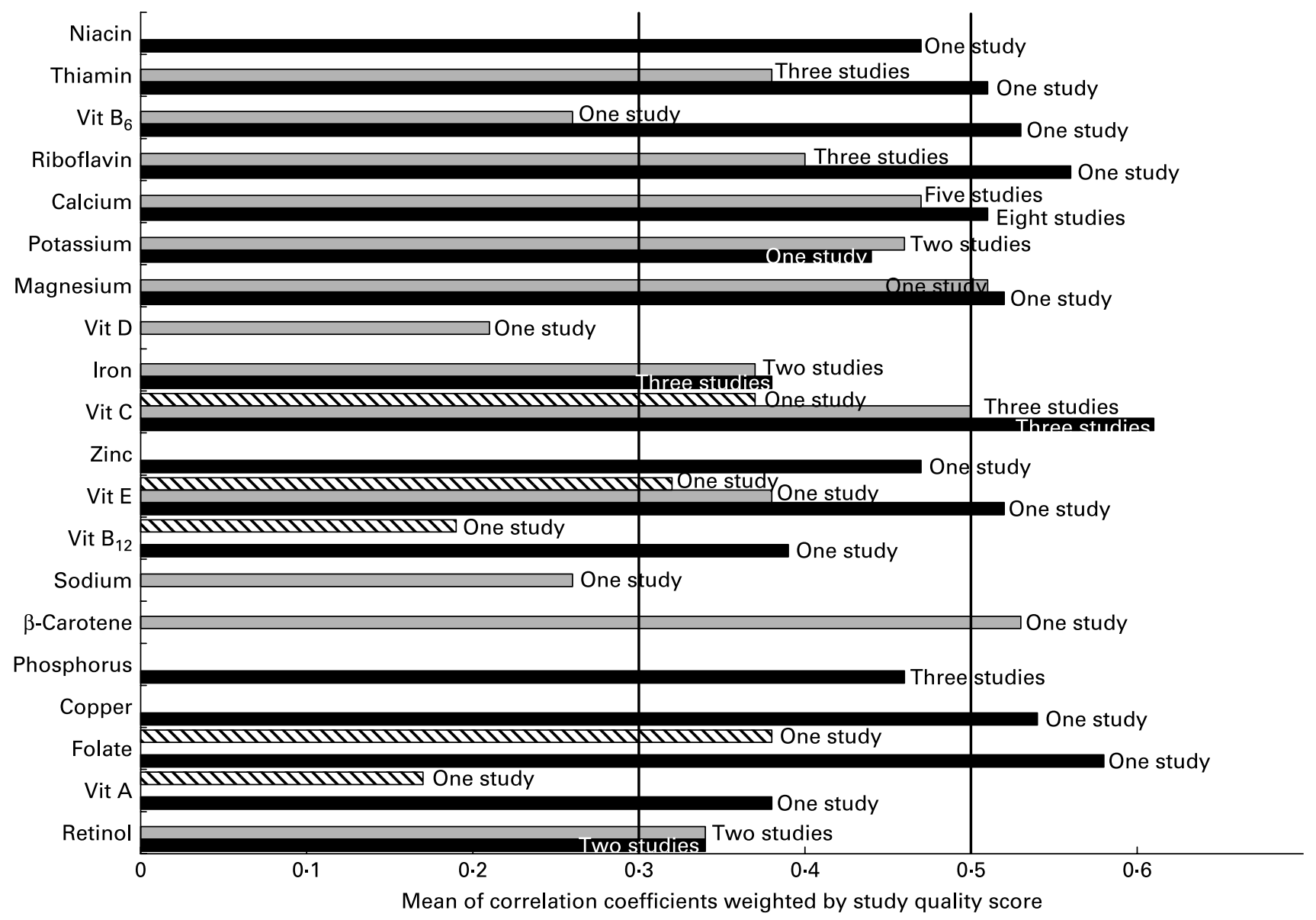

Fig. 5. Validation of FFQ studies that assess micronutrient intake in children (6-12 years) and adolescents (13-10 years) using short-term or long-term dietary instruments or biomarkers as the reference methods. Correlations: poor $(<0.30)$, acceptable $(0.30-0.50)$, good $(0.51-0.70)$ and very good $(>0.70)$. 口, Short-term intake ( $<7 \mathrm{~d}$; three or more studies: vitamin C, iron, calcium, phosphorus); $\square$, long-term intake ( $\geq 7 \mathrm{~d}$; three or more studies: vitamin C, calcium, riboflavin, thiamin); $\mathbb{\mathbb { Q }}$, biomarkers.

recording days (from 1 to $7 \mathrm{~d}$ ) were used as the reference method in a total of four studies.

Comparison of different dietary assessment methods was limited in the adolescent group, due to the fact that there was an insufficient number of studies for each micronutrient that could provide conclusive results (Fig. 3). A good correlation ( $r$ 0.58) was observed, when FFQ were validated applying WDR as the reference method only for $\mathrm{Ca}$. Additionally, when FFQ were validated considering $24 \mathrm{HR}$ as the reference method, the correlation for Ca presented an acceptable correlation $(r$ 0.50). Similar correlations were observed when the validity of Ca intake using the FFQ (including the YAQ) was assessed by comparing them with $\mathrm{Ca}$ intake using the 24HR $(r$ 0.52). There were not enough data to conduct an analysis for the rest of the micronutrients in this population group (Table 5).

Fig. 5 shows only FFQ validation studies that assessed micronutrient intake in children and adolescents, using a short-term ${ }^{(11,13,14,20,25-27,29)}$ or a long-term ${ }^{(16,23,31-33,38)}$ dietary assessment instrument or $\mathrm{BM}$ as the reference $\operatorname{method}^{(23,28,35)}$. In regards to the reference method that reflected short-term intake, good correlations were observed for thiamin, vitamins $\mathrm{B}_{6}, \mathrm{C}, \mathrm{E}$, riboflavin, $\mathrm{Ca}, \mathrm{Mg}, \mathrm{Cu}$ and folate. However, when the reference method used reflected long-term intake, good correlations were observed only for $\mathrm{Mg}$ and $\beta$-carotene. Additionally, when BM were used as the reference method, correlations having a very good classification were not observed. None of the micronutrients analysed showed correlations higher than 0.7 using short or long-term dietary assessment instruments or BM as the reference method. Nevertheless, the results presented in FFQ validation studies using short-term or long-term dietary instruments or $\mathrm{BM}$ as the reference methods based on correlations from less than three studies should be viewed with caution (Fig. 5).

\section{Biomarkers}

A total of five publications analysed $\mathrm{BM}^{(17,22,23,28,35)}$, which were used to validate five FFQ. Some articles presented validation of more than one instrument, of which one study also validated estimated dietary records ${ }^{(17)}$ and two studies validated WDR using $\mathrm{BM}$ as the reference $\operatorname{method}^{(23,28)}$. The BM analysed were: serum markers of $\mathrm{Fe}$ status (ferritin, $\mathrm{Hb})^{(17)}$, plasma levels of vitamins $\mathrm{C}, \mathrm{D}$, retinol, $\beta$-carotene and $\alpha$-tocopherol (vitamin $E)^{(22)}, 24 \mathrm{~h}$ urine $\mathrm{K}^{(23)}$, serum folate and serum vitamin $\mathrm{B}_{12}{ }^{(28)}$ and serum levels of vitamins $\mathrm{C}, \mathrm{A}$ and $\mathrm{E}^{(35)}$. 


\section{Discussion}

In the present review, thirty-two studies ${ }^{(2,8-39)}$ are described. The aim of this analysis was to determine the reliability of methods used to measure the usual intake of vitamins and minerals in infants, children and adolescents and how these were validated. The different studies included in this review were classified according to which reference method was used, those reflecting short-term intake, long-term intake or BM. To rate the different studies, a quality score system was developed by the European micronutrient Recommendations Aligned network. A total score was calculated according to the weighted mean of the correlations that had been adjusted by the quality of the different validation studies, and all the methods were scored into the categories: poor, acceptable, good or very good. Measuring dietary intake in very young children is difficult because of the rapid changes in the food habits of toddlers, the need to rely on parental reporting and the questionable ability of parents to accurately report their child's diet when other caregivers are also involved in feeding the child ${ }^{(22)}$. Adolescents' eating habits are highly influenced by family patterns and habits, their peer group, as well as by their increasing concern with body image. Habits such as meal skipping (particularly breakfast), consuming high-energy foods that are poor in nutrients and the tendency, particularly among girls, to restrict their diet and to go on diets are part of the repertoire of adolescent eating behaviour ${ }^{(20)}$.

\section{Infants}

This group included infants aged 1-23 months. Evidence for the long-term effects of infant nutrition on later health has given impetus to the need for developing methods to assess the diets of infant populations ${ }^{(41)}$. In general, for this group, the daily non-human milk intake in the previous day or week was estimated from the average total volume of bottle-feeds consumed per day; for breast-fed infants, the usual feeding length and number of feeds per day were recorded. In Andersen's study ${ }^{(2)}$, the relative validity of a semi-quantitative FFQ (140-item semi-quantitative FFQ) used in 12-month-old infants was examined (with correlations ranging from 0.18 for vitamin $\mathrm{D}$ to 0.62 for $\mathrm{Ca}$ and $\mathrm{Fe}$ ). This validation study indicated that the semi-quantitative FFQ used in infants overestimated the median intake of nutrients except for $\mathrm{Ca}$ intake. In a sample of fifty infants aged 6 months, Marriott et al. ${ }^{(8)}$ compared the energy and nutrient intakes assessed by a newly developed, interviewer-administered, 34-item infant FFQ with intakes determined from $4 \mathrm{~d}$ WDR. Differences in intakes between methods were observed, and the agreement tended to be lower for breast-fed than for formula-fed infants. The correlation coefficients of this study compared favourably with other FFQ validation studies for young children. Reported correlation coefficients for energy and nutrients were $0.18-0.72$ at 1 year in Andersen's study $^{(2)}$, and $0.26-0.63$ for 1- to 5-year-olds in Blum's study $^{(24)}$. In the latter study, an 84-item FFQ was used, with correlations ranging from 0.31 for $\mathrm{Zn}$ to 0.63 for $\mathrm{Mg}$. Marriott et al. ${ }^{(9)}$ also evaluated the relative validity of a 76-item FFQ for assessing micronutrient intakes in 12-month-old infants. They reported smaller correlations in infants aged 12 months (with correlations ranging from 0.24 for vitamin $B_{12}$ to 0.75 for $\mathrm{Na}$ ) than in the infants aged 6 months (with correlations ranging from 0.55 for $\mathrm{Cu}$ to 0.89 for thiamin). Marshall et al. ${ }^{(21)}$ compared nutrient intakes from beverages in a sample of 6-month-old infants using a 7-item FFQ and obtained coefficients for $\mathrm{Ca}$ and vitamin $\mathrm{D}$ intakes of 0.64 and $0 \cdot 80$, respectively. Similar results were shown for Marriott et al. ${ }^{(8)}$ for these nutrients (Ca, $r 0.78$ and vitamin $\mathrm{D}$, $r=0.83$ ). The ability of the FFQ to accurately rank intakes of energy and all the nutrients in infants is enhanced by the quality and detail of the information collected, which includes information about brands and types of baby foods and milks used $^{(8)}$. In Williams \& Innis ${ }^{(17)}$, parents of 148 infants aged 8-26 months completed a 191-item FFQ and 3d EDR for assessing $\mathrm{Fe}$ nutrition in infants (with correlations ranging from 0.64 for vitamin $\mathrm{C}$ and $\mathrm{Fe}$ to 0.75 for $\mathrm{Ca}$ ). These results showed relative validity of a FFQ in comparison with $3 \mathrm{~d}$ EDR for estimating $\mathrm{Fe}$ intakes in a group of infants and suggested that while dietary assessments can serve as useful research tools to assess nutrient intakes in 8- to 26-month-old infants, they have limited value as tools to identify infants at risk of Fe deficiency.

\section{Preschool children}

In this review, the preschool children group included children aged 2-5 years. Many factors contribute to making intake assessment in this age group difficult: preschool children eat small amounts of food at frequent intervals, they are not able to complete questionnaires and their food habits and nutrient intakes may rapidly change ${ }^{(42,43)}$. In Huybrechts et al. ${ }^{(15)}$, the relative validity of a 47 -item FFQ for measuring preschool children's usual Ca intake was assessed using parents or guardians as a proxy, and EDR were used as the reference method. Based on the comparison of means, the FFQ underestimated the mean $\mathrm{Ca}$ intake measured by the EDR. These findings are in contrast to the findings of another study ${ }^{(26)}$, which reported overestimates of actual $\mathrm{Ca}$ intakes in young children (3-6 years) using a 35-item FFQ. Andersen et al. ${ }^{(18)}$ presented the relative validity of food and nutrient intakes estimated by the 125-item FFQ against intakes from $7 \mathrm{~d}$ WDR applied in the nation-wide survey among 2-year-old children in Norway. The correlations in this study were also lower than those observed by Blum et al. ${ }^{(24)}$ However, the correlation coefficients for nutrient density values estimated from the two methods were much higher than those seen for absolute nutrient intakes, and for ten out of sixteen nutrients, the correlations were $>0.50$. The average correlation (median $r 0.52$ ) for nutrient density was similar to that observed among 12-month-old Norwegian infants (median $r \quad 0.50)^{(2)}$. The agreement across quartiles between the two methods was on average (median) $36 \%$, which is similar to the results observed among 12-month-old infants $(38 \%)^{(2)}$. In Andersen's study ${ }^{(18)}$, agreement across quartiles increased when using nutrient density. Other authors studied 224 preschool children and obtained dietary data by interviewing the child's mother ${ }^{(37)}$. The present study was performed to determine the utility of the Willett semi-quantitative FFQ for assessing habitual diets of preschool children. Results reported associations between nutrient intakes estimated by the FFQ and $24 \mathrm{~h}$ recalls, with correlations (adjusted 
for within-person variability and non-differential measurement error) in the range of $0 \cdot 20-0 \cdot 60^{(37)}$. Blum et al. ${ }^{(24)}$ demonstrated that past dietary intake of children aged 1-5 years could be measured reasonably well with an 84-item FFQ completed by child's parent or guardian. Correlation coefficients between the dietary intakes assessed by the two methods ranged from 0.26 for fibre to 0.63 for $\mathrm{Mg}$. All but three nutrients (protein, dietary fibre and $\mathrm{Zn}$ ) had correlations of 0.47 or higher. After adjusting for energy intake and withinperson variation, the average correlation was 0.52 .

\section{Children}

This group included children aged 6-12 years. The food and nutrient intake of children is important not only for growth and development but also for present and future health, including the prevention of chronic diseases in adulthood ${ }^{(44,45)}$. To succeed in nutrition monitoring and epidemiologic research among large groups of children, it is necessary to have a dietary assessment method that is both valid and functional with young age groups ${ }^{(12)}$. Almost all of the reviewed validity and reliability studies in children younger than age 9 included adult assistance in providing information on the child's intake. In general, the reviews concluded that children generally have difficulty in estimating portion sizes ${ }^{(46)}$. Räsänen ${ }^{(39)}$ compared the $24 \mathrm{~h}$ recall method with the diet history method as used in a food consumption survey for children. The correlation coefficients between the values obtained by the $24 \mathrm{~h}$ recalls and the diet history method varied from 0.20 (vitamin A) to 0.50 (energy). The history method used in this study gave consistently higher mean values than the $24 \mathrm{~h}$ recalls. Lytle et al. ${ }^{(36)}$ found correlations for nutrient intake between 0.45 and 0.79 when comparing observed and recalled food intake in 8- to 10-year-old children. These are within the same range or somewhat higher than results obtained in Lillegaard's study ${ }^{(12)}$, which evaluated intake of energy, macro- and micronutrients assessed from pre-coded food diaries and by using $4 \mathrm{~d}$ WDR as the reference method in 9-year-old Norwegians. The pre-coded food diary is scanable and is developed to simplify the work of the respondents as well as of the researcher. In Belgium, a self-administered computer dietary assessment program 'Young Adolescents Nutrition Assessment Computer' was developed, based on the concept of the 24HR. The Young Adolescents Nutrition Assessment Computer is a computer program designed for use with children and adolescents aged 11 years and over ${ }^{(47)}$. Vereecken et al. ${ }^{(47)}$ assessed the relative validity of the computerised $24 \mathrm{~h}$ recall Young Adolescents Nutrition Assessment Computer comparing results with a $1 \mathrm{~d}$ EDR and $24 \mathrm{~h}$ recall; the Spearman's correlations obtained for the $24 \mathrm{~h}$ recall (ranging from 0.44 to 0.86 ) were comparable to those observed by Lytle et al. ${ }^{(36)}$ between $24 \mathrm{~h}$ recalled and observed nutrient intake of third-grade children (ranging between 0.45 and 0.79). In the study of Van Horn et al. ${ }^{(48)}$, the correlations were slightly higher (0.64-0.96) using electronic methods such as telephone recalls and tape-recorded dietary records. Bellù et al. ${ }^{(30)}$ reported on the ability of a 116-item FFQ to estimate the mean nutrient intake at the population level for an Italian paediatric population (mean age $=9.3$ years) comparing results with a $24 \mathrm{~h}$ recall. Moderate concordance between the two methods was found. Similar results were presented in by Stein et al. ${ }^{(37)}$ In another study, Bellù et al. ${ }^{(32)}$ tested the validity for the assessment of individual nutrient intake by a 116-item FFQ for Northern Italian school children comparing results with $7 \mathrm{~d}$ EDR. In this study, the Pearson's correlation coefficients ranged between 0.30 and 0.58 , and for some nutrients (vitamins $\mathrm{A}$ and $\mathrm{B}_{6}$ ) the correlation was found to be low.

\section{Adolescents}

For the present paper, the adolescent age group included youth aged $13-18$ years. Adolescents are a group whose eating habits are characterised by factors such as irregular meals, snacking and meal-skipping. These eating habits are not easily conducive to diet assessment by a prospective methodology ${ }^{(23)}$. Slater et al. ${ }^{(20)}$ compared the 76-item FFQ developed for adolescents to data obtained from $24 \mathrm{~h}$ recalls, and similar values for energy, carbohydrates, total fat and $\mathrm{Ca}$ intake were observed, suggesting a high consistency in estimating these nutrients. However, there was a significant difference for seven remaining nutrients (protein, polyunsaturated fat, dietary fibre, cholesterol, retinol, vitamin $\mathrm{C}$ and $\mathrm{Fe}$ ). Higher values (mean $r$ 0.57) were observed with unadjusted correlations compared with results obtained by Rockett et al. ${ }^{(29)}$ (mean $r$ 0.39) and Field et al. ${ }^{(25)}(0.27$ for $\mathrm{Ca}, 0.25$ for $\mathrm{P}, 0.20$ for $\mathrm{Fe}, 0.19$ for vitamin $\mathrm{C}$ ). The YAQ is a youth-friendly FFQ that allows 9- to 18-year-old adolescents to report on their own diet ${ }^{(49)}$. Rockett et al. ${ }^{(29)}$ evaluated the questionnaire's validity by comparing the nutrient scores of the YAQ with those from the average of three $24 \mathrm{~h}$ recalls. Similar mean nutrients were found by both the methods. Correlation coefficients between the mean energy-adjusted nutrients computed by the two methods ranged from 0.21 for $\mathrm{Na}$ to 0.58 for folate. After correction for within-person error, the average correlation coefficient was $0 \cdot 54$, similar to that found for adults ${ }^{(50)}$. In the validation study developed by Mølgaard et al. ${ }^{(27)}$, twentythree children aged 13-14 years filled in an 88-item FFQ designed to assess $\mathrm{Ca}$, protein and $\mathrm{P}$ intakes and kept $3 \mathrm{~d}$ WDR. Spearman rank correlations between nutrient intake values from the FFQ and WDR were 0.56-0.62 (mean 0.60). Another study showed similar findings for $\mathrm{Ca}$ intake $(r 0.54)^{(33)}$ using $7 \mathrm{~d}$ WDR as the reference method.

\section{Biomarkers}

Williams \& Innis ${ }^{(17)}$ showed the validity of nutrient intake estimates using a 191-item FFQ that was assessed by comparing data with biochemical measures of Fe status in infants. These results presented weak correlations for assessment methods (acceptable classification for FFQ $v$. BM $r 0.33$ and poor classification for a $3 \mathrm{~d}$ EDR $v$. BM $r$ 0.19). This is the only study presented in this review that analyses biochemical measures of $\mathrm{Fe}$ status in infants and possibly, the lower correlation coefficients between $\mathrm{Fe}$ intake and serum ferritin observed in this study, as compared to previous studies with older subjects, involve factors inherent to studies in infants but not in older children and adults ${ }^{(51)}$. Lietz et al. ${ }^{(23)}$ reported on the correlation coefficient between urinary $\mathrm{K}$ and dietary intake from a 130-item FFQ $(r-0.04)$ and from a $7 \mathrm{~d}$ WDR ( $r$ 0.78) in fifty children between 11 and 13 years. The latter 
correlation was higher than correlations reported in a previous study ${ }^{(52)}$.

Green et al. ${ }^{(28)}$ validated a 116-item FFQ and a $3 \mathrm{~d}$ WDR by comparing nutrient intake estimates using these methods with serum folate and serum vitamin $B_{12}$ concentrations in 105 females aged 16-19 years. Using serum folate concentration as the sole biochemical criterion, it appeared that the $3 \mathrm{~d}$ WDR was superior to the FFQ as a tool to predict folate intakes. The correlations between folate intakes and serum folate as determined by $3 \mathrm{~d}$ WDR (adjusted $r$ 0.65) showed higher correlations than those determined by FFQ ( $r$ 0.48; supplement users were included in these analyses). Vitamin $\mathrm{B}_{12}$ intake as determined by either of the dietary methods showed only a modest association with serum vitamin $B_{12}$ concentrations, when supplement users were included in the analyses ( $3 \mathrm{~d}$ WDR $r$ 0.32; FFQ $r$ 0.25). Another author ${ }^{(53)}$ using a FFQ reported a correlation of $r 0.60$ between folate intakes and plasma folate concentrations and a correlation of $r 0.35$ between vitamin $\mathrm{B}_{12}$ intakes and serum vitamin $\mathrm{B}_{12}$ concentrations in a group of 139 adults aged 40-83 years.

The correlations observed by Parrish et al. ${ }^{(22)}$ compared the reported dietary intake in infants and preschool children using a 111-item FFQ with plasma concentrations of the micronutrient. The highest correlation between plasma concentrations and dietary intake as measured by the FFQ was for vitamin $C(r 0 \cdot 51)$, and the correlations were weak or absent for vitamin $\mathrm{D}$, retinol and $\beta$-carotene. Byers et al. ${ }^{(35)}$ administered a 111-item FFQ to ninety-seven parents of children aged 6-10 years to evaluate their children's usual dietary intake and compared results with children's serum levels of vitamins $\mathrm{C}, \mathrm{A}$ and $\mathrm{E}$. The correlations observed in this study between circulating levels of vitamin $C(r \quad 0 \cdot 37)$ and vitamin $\mathrm{E}(r 0.32)$, and the parent's reports of their children's diets were similar to those observed in dietary validation studies conducted in adults ${ }^{(54)}$. However, it is important to keep in mind that the results presented in FFQ validation studies using BM as the reference method based on correlations from only one or two studies should be interpreted with caution (Figs. 4 and 5).

\section{Conclusion}

When comparing different validation methods in infants, the reviewed studies showed that the FFQ was the method used in all the studies to assess micronutrient intake in infants. Different FFQ were validated for which wide variations in the number of food items were observed (7-191 items). The ability of the FFQ to accurately rank intakes of energy and all the nutrients in infants is enhanced by the quality and detail of the information collected, which includes information about brands and types of baby foods and milks used $^{(8)}$. The WDR used as the reference method for evaluating FFQ present better correlations for several micronutrients than other methods in this population group. However, we must emphasise that this result is probably due to the fact that $57 \%$ of the studies analysed in infants used WDR as the reference method. Vitamin D and vitamin E intake analysed using FFQ $v$. WDR showed acceptable correlations, and good correlations were observed for vitamin $\mathrm{C}$, thiamin, riboflavin, $\mathrm{Ca}$ and $\mathrm{Fe}$. For the rest of the micronutrients, there were insufficient data to conduct an analysis (three or more studies were needed for each micronutrient).

In the preschool children cluster, different FFQ were validated for which wide variations in the number of food items were observed ( $7-125$ items).The FFQ was used as the method to assess micronutrient intake in $80 \%$ of the studies in preschool children. Comparison of different dietary assessment methods was difficult in this group, as there were not enough studies to analyse for each micronutrient. Except for the measurement of $\mathrm{Ca}$ intake, acceptable correlations $(r$ 0.50) were observed when FFQ were validated applying WDR and EDR as the reference methods. This age group typically eat more frequently, and since $50 \%$ of the preschool children consume two meals outside of their homes, parents may not be able to accurately describe what was actually consumed ${ }^{(34)}$.

The results presented in the FFQ validation studies for infants and preschool children, using long-term dietary instruments or BM as the reference methods were based on only one or two correlation studies and as such, there were insufficient data to reach robust conclusions. In regards to the reference method that reflected short-term intake in the infants and preschool children group, good correlations were observed for niacin, thiamin, vitamins $\mathrm{B}_{6}, \mathrm{D}, \mathrm{C}, \mathrm{E}$, riboflavin, $\mathrm{Ca}, \mathrm{K}, \mathrm{Mg}$, $\mathrm{Fe}$, and $\mathrm{Zn}$ (with mean weighted correlations ranging from 0.55 for vitamin $\mathrm{E}$ to 0.69 for niacin). For the analysis of these micronutrients, data from three or more studies were included (the study quality scores ranging from 2.5 to $5 \cdot 5$ ).

For the children group, $65 \%$ of the reviewed studies used FFQ to assess micronutrient intake for which wide variations in the number of food items were observed (10-175 items). When comparing different validation methods in this group, only an acceptable correlation ( $r$ 0.50) was observed for measuring of $\mathrm{Ca}$ intake, when FFQ were validated applying WDR and EDR as the reference methods. Similar correlations were observed when the validity of $\mathrm{Ca}$ intake estimates using the FFQ (including YAQ) was assessed by comparing them with the Ca intake estimates using a 24HR ( $r$ 0.49). For the other micronutrients, there were insufficient data to compare.

Assessing the validation of different dietary assessment methods in adolescents was difficult as there were not enough studies to compare for each micronutrient. A good correlation ( $r$ 0.58) was observed when FFQ were validated applying WDR as the reference method only for Ca intake. Additionally, when FFQ were validated using a $24 \mathrm{HR}$ as the reference method, correlations for $\mathrm{Ca}$ presented acceptable values $(r 0.50)$. In the adolescent group, $80 \%$ of the reviewed studies used FFQ to assess micronutrient intake for which wide variations in the number of food items were observed (10-190 items).

There were not enough data to contrast FFQ studies that assessed micronutrient intake in children and adolescents using long-term dietary instruments (except for thiamin, riboflavin, $\mathrm{Ca}$ and vitamin $\mathrm{C}$ intake that showed acceptable correlations) or BM as the reference methods. (Only one or two studies, thus being insufficient to reach strong conclusions). Regarding the reference method that reflected short-term intake in children and adolescents, good correlations were observed only for vitamin $\mathrm{C}(r$ 0.61) and $\mathrm{Ca}$ $(r$ 0.51). In the analysis of these micronutrients, data from three or more studies were included (the study quality scores ranging from 2 to $4 \cdot 5$ ). 
In the studies reviewed, FFQ comprised the dietary method that was most utilised to assess the micronutrient intake in these groups, in which it is of utmost importance to recognise methodological aspects such as food composition databases used for analysis, portion size assessment and the time periods between the two dietary assessment methods ${ }^{(23)}$. On the other hand, dietary assessment in children and adolescents using electronic methods for deriving self-reports, such as the tape-recorded food record or the computer dietary assessment program, may help improve compliance and frequency of record keeping in these age groups ${ }^{(48)}$.

The micronutrients analysed in this review using $\mathrm{BM}$ as the reference method were: vitamins $\mathrm{A}, \mathrm{C}, \mathrm{D}, \mathrm{E}$, and $\mathrm{B}_{12}$, retinol, $\beta$-carotene, folate and $\mathrm{K}$. In general, the best correlations were observed when the validity of nutrient intake estimates using WDR was assessed by comparing them with micronutrient serum levels. Validation of FFQ studies that assess micronutrient intake in the infant and preschool children group using BM as the reference method presented good correlations only for vitamin C. Poor rankings $(r<0 \cdot 3)$ were observed for retinol, $\beta$-carotene and $\mathrm{K}$, and acceptable rankings were observed only for vitamin $\mathrm{E}$ and $\mathrm{Fe}$. In the children and adolescent group, the validation of FFQ studies that assess micronutrient intake comparing them with biochemical measures of micronutrient status presented poor correlations for vitamins $A$ and $B_{12}$ and acceptable correlations for folate, vitamins $\mathrm{E}$ and $\mathrm{C}$. However, we must emphasise once more that the data presented in FFQ validation studies using BM as the reference method showed correlations from only one or two studies; as such, this information should be viewed with caution. Including supplement users generally improved the correlations between micronutrients intakes estimated by either of the dietary intake methods and their respective biochemical indices. Nutrient BM are appealing as a comparison method because measurement errors are uncorrelated with reporting errors $^{(55)}$. However, given that blood concentrations may be a result of absorption and metabolism and not only reflecting intake, nutrient BM may not always be an appropriate method of comparison ${ }^{(22)}$.

\section{Acknowledgements}

The studies reported herein have been carried out within the European micronutrient Recommendations Aligned Network of Excellence (www.eurreca.org), financially supported by the Commission of the European Communities, specific Research, Technology and Development (RTD) Programme Quality of Life and Management of Living Resources, within the Sixth Framework Programme, contract no 036196. This report does not necessarily reflect the Commission's views or its future policy in this area. A. O.-A. wrote the first manuscript; A. O.-A., P. H.-S. and A. S.-V. contributed to the planning of the search and analysed the articles included in this review; M. M. analysed the articles included in this review; L. P.-Q. and M. M. revised and discussed previous drafts; L. S.-M. contributed to the planning of the search, decided the analysis and presentation of the results and created the quality assessment tool of the articles, and revised and discussed previous drafts. There are no conflicts of interest to report. The authors of the present paper would like to thank Dr Margaret Ashwell, Dr Janet Lambert,
Dr Adriënne Cavelaars, Dr Olga Souverein and Mrs Sandra Crispim for their technical contribution and advice to this publication. The authors also thank the Health Sciences Library of the University of Las Palmas de Gran Canaria, Lourdes Ribas, and Nuria Melián for their contribution in the collection of articles analysed in this review. Special thanks to Joy Ngo, RD from the Nutrition Research Foundation (FIN) for her help in editing the English version of the manuscript.

\section{References}

1. Mascarenhas MR, Zemel B \& Stallings VA (1998) Nutritional assessment in paediatrics. Nutrition 14, 105-115.

2. Andersen LF, Lande B, Arsky GH, et al. (2003) Validation of a semi-quantitative food-frequency questionnaire used among 12-month-old Norwegian infants. Eur J Clin Nutr 57, 881-888.

3. Baranowski T \& Domel SB (1994) A cognitive model of children's reporting of food intake. Am J Clin Nutr 59, S212-S217.

4. Livingstone MB \& Robson PJ (2000) Measurement of dietary intake in children. Proc Nutr Soc 59, 279-293.

5. Sobo EJ \& Rock CL (2001) 'You ate all that!?': caretaker-child interaction during children's assisted dietary recall interviews. Med Anthropol Q 15, 222-244.

6. Frank GC (1997) Methodological issues regarding eating behavior of high-risk adolescents. Ann N Y Acad Sci 817, 66-82.

7. Ashwell M, Lambert JP, Alles MS, et al. (2008) How we will produce the evidence-based EURRECA toolkit to support nutrition and food policy. Eur J Nutr 47, Suppl. 1, 2-16.

8. Marriott LD, Robinson SM, Poole J, et al. (2008) What do babies eat? Evaluation of a food frequency questionnaire to assess the diets of infants aged 6 months. Public Health Nutr 11, 751-756.

9. Marriott LD, Inskip HM, Borland SE, et al. (2008) What do babies eat? Evaluation of a food frequency questionnaire to assess the diets of infants aged 12 months. Public Health Nutr 12, 967-972.

10. Holmes B, Dick K \& Nelson M (2008) A comparison of four dietary assessment methods in materially deprived households in England. Public Health Nutr 11, 444-456.

11. Moore M, Braid S, Falk B, et al. (2007) Daily calcium intake in male children and adolescents obtained from the rapid assessment method and the 24-hour recall method. Nutr J 6, 24-28.

12. Lillegaard IT, Løken EB \& Andersen LF (2007) Relative validation of a pre-coded food diary among children, underreporting varies with reporting day and time of the day. Eur $J$ Clin Nutr 61, 61-68.

13. Magkos F, Manios Y, Babaroutsi E, et al. (2006) Development and validation of a food frequency questionnaire for assessing dietary calcium intake in the general population. Osteoporos Int 17, 304-312.

14. Harnack LJ, Lytle LA, Story M, et al. (2006) Reliability and validity of a brief questionnaire to assess calcium intake of middle-school-aged children. J Am Diet Assoc 106, 1790-1795.

15. Huybrechts I, De Bacquer D, Matthys C, et al. (2006) Validity and reproducibility of a semi-quantitative food-frequency questionnaire for estimating calcium intake in Belgian preschool children. Br J Nutr 95, 802-816.

16. Bertoli S, Petroni ML, Pagliato E, et al. (2005) Validation of food frequency questionnaire for assessing dietary macronutrients and calcium intake in Italian children and adolescents. $J$ Pediatr Gastroenterol Nutr 40, 555-560.

17. Williams PL \& Innis SM (2005) Food frequency questionnaire for assessing infant iron nutrition. Can J Diet Pract Res 66, $176-182$. 
18. Andersen LF, Lande B, Trygg K, et al. (2004) Validation of a semi-quantitative food-frequency questionnaire used among 2-year-old Norwegian children. Public Health Nutr 7, 757-764.

19. Andersen LF, Lande B, Trygg K, et al. (2004) Validation of a semi-quantitative food-frequency questionnaire used among 2-year-old Norwegian children - Corrigendum. Public Health Nutr 12, 1026-1027.

20. Slater B, Philippi ST, Fisberg RM, et al. (2003) Validation of a semi-quantitative adolescent food frequency questionnaire applied at a public school in São Paulo, Brazil. Eur J Clin Nutr 57, 629-635.

21. Marshall TA, Eichenberger Gilmore JM, Broffitt B, et al. (2003) Relative validation of a beverage frequency questionnaire in children ages 6 months through 5 years using 3-day food and beverage diaries. Am Diet Assoc 103, 714-720.

22. Parrish LA, Marshall JA, Krebs NF, et al. (2003) Validation of a food frequency questionnaire in preschool children. Epidemiology 14, 213-217.

23. Lietz G, Barton KL, Longbottom PJ, et al. (2002) Can the EPIC food-frequency questionnaire be used in adolescent populations? Public Health Nutr 5, 783-789.

24. Blum RE, Wei EK, Rockett HR, et al. (1999) Validation of a food frequency questionnaire in Native American and Caucasian children 1 to 5 years of age. Matern Child Health J 3, 167-172.

25. Field AE, Peterson KE, Gortmaker SL, et al. (1999) Reproducibility and validity of a food frequency questionnaire among fourth to seventh grade inner-city school children: implications of age and day-to-day variation in dietary intake. Public Health Nutr 2, 293-300.

26. Taylor RW \& Goulding A (1998) Validation of a short food frequency questionnaire to assess calcium intake in children aged 3 to 6 years. Eur J Clin Nutr 52, 464-465.

27. Mølgaard C, Sandström B \& Michaelsen KF (1998) Evaluation of a food frequency questionnaire for assessing of calcium, protein and phosphorus intakes in children and adolescents. Scand J Nutr 42, 2-5.

28. Green TJ, Allen OB \& O'Connor DL (1998) A three-day weighed food record and a semiquantitative food-frequency questionnaire are valid measures for assessing the folate and vitamin B-12 intakes of women aged 16 to 19 years. J Nutr 128, $1665-1671$.

29. Rockett HR, Breitenbach M, Frazier AL, et al. (1997) Validation of a youth/adolescent food frequency questionnaire. Prev Med 26, 808-816.

30. Bellù R, Riva E, Ortisi MT, et al. (1996) Validity of a food frequency questionnaire to estimate mean nutrient intake of Italian school children. Nutr Res 16, 197-200.

31. Arnold JE, Rohan T, Howe G, et al. (1995) Reproducibility and validity of a food-frequency questionnaire designed for use in girls age 7 to 12 years. Ann Epidemiol 5, 369-377.

32. Bellù R, Ortisi MT, Riva E, et al. (1995) Validity assessment of a food frequency questionnaire for school-age children in Northern Italy. Nutr Res 15, 1121-1128.

33. Andersen LF, Nes M, Lillegaard IT, et al. (1995) Evaluation of a quantitative food frequency questionnaire used in a group of Norwegian adolescents. Eur J Clin Nutr 49, 543-554.

34. Iannotti RJ, Zuckerman AE, Blyer EM, et al. (1994) Comparison of dietary intake methods with young children. Psychol Rep 74, 883-889.

35. Byers T, Trieber F, Gunter E, et al. (1993) The accuracy of parental reports of their children's intake of fruits and vegetables: validation of a food frequency questionnaire with serum levels of carotenoids and vitamins C, A, and E. Epidemiology 4, 350-355.

36. Lytle LA, Nichaman MZ, Obarzanek E, et al. (1993) Validation of 24-hour recalls assisted by food records in third-grade children. The CATCH Collaborative Group. J Am Diet Assoc 93, $1431-1436$.

37. Stein AD, Shea S, Basch CE, et al. (1992) Consistency of the Willett semiquantitative food frequency questionnaire and 24-hour dietary recalls in estimating nutrient intakes of preschool children. Am J Epidemiol 15, 667-677.

38. Jenner DA, Neylon K, Croft S, et al. (1989) A comparison of methods of dietary assessment in Australian children aged 11-12 years. Eur J Clin Nutr 43, 663-673.

39. Räsänen L (1979) Nutrition survey of Finnish rural children. VI. Methodological study comparing the 24-hour recall and the dietary history interview. Am J Clin Nutr 32, 2560-2567.

40. Treadwell JR, Tregear SJ, Reston JT, et al. (2006) A system for rating the stability and strength of medical evidence. BMC Med Res Methodol 6, 52-70.

41. Owen CG, Martin RM, Whincup PH, et al. (2005) Effect of infant feeding on the risk of obesity across the life course: a quantitative review of published evidence. Pediatrics 115, $1367-1377$.

42. Hertzler AA, Bowens J \& Hull S (1993) Preschoolers' reporting of food habits. J Am Diet Assoc 93, 1159-1161.

43. Stein AD, Shea S, Basch CE, et al. (1991) Variability and tracking of nutrient intakes of preschool children based on multiple administrations of the 24-hour dietary recall. Am J Epidemiol 134, 1427-1437.

44. Berenson GS (2002) Childhood risk factors predict adult risk associated with subclinical cardiovascular disease. The Bogalusa Heart Study. Am J Cardiol 90, 3L-7L.

45. McGill HC Jr, McMahan CA, Herderick EE, et al. (2000) Origin of atherosclerosis in childhood and adolescence. Am J Clin Nutr 72, Suppl. 5, 1307-1315.

46. Cypel YS, Guenther PM \& Petot GJ (1997) Validity of portionsize measurement aids: a review. J Am Diet Assoc 97, 289-292.

47. Vereecken CA, Covents M, Matthys C, et al. (2005) Young adolescents' nutrition assessment on computer (YANA-C). Eur $J$ Clin Nutr 59, 658-667.

48. Van Horn LV, Gernhofer N, Moag-Stahlberg A, et al. (1990) Dietary assessment in children using electronic methods: telephones and tape recorders. J Am Diet Assoc 90, 412-416.

49. Lamb MM, Ross CA, Brady HL, et al. (2007) Comparison of children's diets as reported by the child via the Youth/Adolescent Questionnaire and the parent via the Willett food-frequency questionnaire. Public Health Nutr 10, 663-670.

50. Rimm EB, Giovannucci EL, Stampfer MJ, et al. (1992) Reproducibility and validity of an expanded self-administered semiquantitative food frequency questionnaire among male health professionals. Am J Epidemiol 135, 1114-1126.

51. Fomon SJ, Ziegler EE, Nelson SE, et al. (1981) Cow milk feeding in infancy: gastrointestinal blood loss and iron nutritional status. J Pediatr 98, 540-545.

52. McKeown NM, Day NE, Welch AA, et al. (2001) Use of biological markers to validate self-reported dietary intake in a random sample of the European Prospective Investigation into Cancer United Kingdom Norfolk cohort. Am J Clin Nutr 74, $188-196$.

53. Jacques PF, Sulsky SI, Sadowski JA, et al. (1993) Comparison of micronutrient intake measured by a dietary questionnaire and biochemical indicators of micronutrient status. Am J Clin Nutr 57, $182-189$.

54. Coates RJ, Eley JW, Block G, et al. (1991) An evaluation of a food frequency questionnaire for assessing dietary intake of specific carotenoids and vitamin $\mathrm{E}$ among low-income black women. Am J Epidemiol 134, 658-671.

55. Willett WC, Sampson L, Stampfer MJ, et al. (1985) Reproducibility and validity of a semiquantitative food frequency questionnaire. Am J Epidemiol 122, 51-65. 